Kardiologe 2021 · 15:595-641

https://doi.org/10.1007/s12181-021-00509-1

Angenommen: 17. August 2021

Online publiziert: 18. Oktober 2021

(c) Deutsche Gesellschaft für Kardiologie - Herzund Kreislaufforschung e.V. Published by Springer Medizin Verlag $\mathrm{GmbH}$, ein Teil von Springer Nature - all rights reserved 2021

\section{Manual zur Indikation und Durchführung spezieller echokardiographischer Anwendungen}

Andreas Hagendorff ${ }^{1}$ (D) - Andreas Helfen ${ }^{2}$ (D) - Frank A. Flachskampf ${ }^{3}$ (D) Sebastian Ewen ${ }^{4}$ (D) - Sebastian Kruck ${ }^{5} \cdot$ Karl La Rosée $^{6} \cdot$ Jan Knierim ${ }^{7}$. Jens-Uwe Voigt ${ }^{8}(\mathrm{D}) \cdot$ Felix Kreidel $^{9} \cdot$ Wolfgang Fehske ${ }^{10}$ (D) $\cdot$ Roland Brandt $^{11}$. Ralf Zahn ${ }^{12,13}$ (iD $\cdot$ Fabian Knebel ${ }^{14,15}$ (iD

${ }^{1}$ Klinik und Poliklinik für Kardiologie, Universitätsklinikum Leipzig, Leipzig, Deutschland; ${ }^{2}$ Medizinische Klinik I, Katholisches Klinikum Lünen Werne GmbH St. Marien-Hospital Lünen, Lünen, Deutschland; ${ }^{3}$ Department of Medical Sciences, Universität Uppsala, und Klinisk fysiologi och kardiologi, Uppsala University Hospital, Uppsala, Schweden; ${ }^{4}$ Klinik für Innere Medizin III - Kardiologie, Angiologie und Internistische Intensivmedizin, Universitätsklinikum des Saarlandes, Homburg/Saar, Deutschland; ${ }^{5}$ Cardio Centrum Ludwigsburg Bietigheim, Ludwigsburg, Deutschland; ${ }^{6}$ Gemeinschaftspraxis Dr. La Rosée \& Prof. Dr. Müller, Bonn, Deutschland; ${ }^{7}$ Klinik für Herz-, Thorax- und Gefäßchirurgie, Deutsches Herzzentrum Berlin, Berlin, Deutschland; ${ }^{8}$ Department of Cardiovascular Diseases, University Hospital Gasthuisberg und Department of Cardiovascular Sciences, Cath. University Leuven, Leuven, Belgien; ${ }^{9}$ Zentrum für Kardiologie, Universitätsmedizin Mainz, Mainz, Deutschland; ${ }^{10} \mathrm{Klinik}$ III für Innere Medizin, Universitätsklinikum Köln - Herzzentrum, Universität zu Köln, Köln, Deutschland; " Abteilung für Kardiologie, Kerckhoff Klinik GmbH, Bad Nauheim, Deutschland; ${ }^{12}$ Medizinische Klinik B - Abteilung für Kardiologie, Klinikum der Stadt Ludwigshafen gGmbH, Ludwigshafen am Rhein, Deutschland; ${ }^{13}$ Kommission für Klinische Kardiovaskuläre Medizin, Deutsche Gesellschaft für Kardiologie, Düsseldorf, Deutschland; ${ }^{14}$ Medizinische Klinik mit Schwerpunkt Kardiologie und Angiologie, Charité Universitätsmedizin Berlin, Campus Mitte, Berlin, Deutschland; ${ }^{15}$ Sana Klinikum Lichtenberg, Berlin, Deutschland

\section{Zusatzmaterial online}

Die Online-Version dieses Beitrags (https:// doi.org/10.1007/s12181-021-00509-

1) enthält ein Abkürzungs-, Tabellen-, Abbildungsverzeichnis.

R.Zahnhatandiesem BeitragfürdieKommission für Klinische Kardiovaskuläre Medizin der DGK mitgewirkt.

Der Verlag veröffentlicht die Beiträge in der von den Autor*innen gewählten Genderform. Die Verwendung einer angemessenen gendergerechten Sprache, um Menschen in ihrer Vielfalt wertschätzend anzusprechen, wird begrüßt.

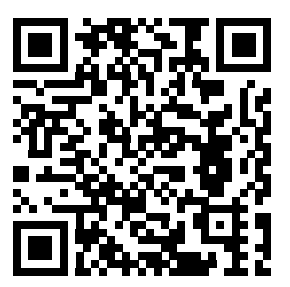

QR-Code scannen \& Beitrag online lesen

\section{Einleitung}

Das folgende Manual zur speziellen Indikationsstellungen und Durchführung erweiterter Techniken der Echokardiographie ist die Ergänzung des ersten Manuals zur Indikationsstellung und Durchführung der transthorakalen Echokardiographie $[8,34]$ und gibt auf Grundlage der aktuellen wissenschaftlichen Literatur den Konsens der Arbeitsgruppen „kardiovaskulärer Ultraschall“ (AG05) der Deutschen Gesellschaft für Kardiologie (DGK) und der Arbeitsgruppe Echokardiographie der Deutschen Gesellschaft für Ultraschall in der Medizin (DEGUM) wieder.

Die konventionelle Echokardiographie umfasst methodisch die M-Mode, 2-dimensionale (2D), ,pulsed wave", „, continuous wave" und farbkodierte Dopplerechokardiographie (PW-, CW- und Farbdoppler). Etablierte zusätzliche echokardiographische Modalitäten wie die transösophageale Echokardiographie, die Rechts- und
Linksherz-Kontrastechokardiographie und die Stressechokardiographie werden speziell durch die multiplane und 3-dimensionale (3D) Echokardiographie ergänzt, die in modernen Echokardiographielaboren derzeit nahezu bei allen echokardiographischen Anwendungen zum Einsatz kommt. Entwicklungen wie die myokardiale Kontrastechokardiographie oder das Blood Flow Speckle Tracking haben sich dagegen noch nicht in der Klinik etabliert. Ebenfalls erweitert das sog. „Postprocessing" echokardiographischer Bilddatensätze sowohl eine Verbesserung der Analyse von Herz- und Klappenmorphologie (Optimierung des 3D-Renderings und Schnittbildanalysen innerhalb eines 3DDatensatzes) als auch eine reproduzierbare und objektive Analyse der Herzfunktion (Deformationsimaging mit gepulsten und farbkodierten Gewebe-Doppler oder 2DSpeckle- bzw. 3D-Voxel-Tracking).

Diese neuen Möglichkeiten der speziellen Echokardiographie müssen im 
Hinblick auf methodische Aspekte sowie spezielle Indikationsstellungen bei besonderen Zielgruppen erörtert werden. Die Gliederung des folgenden Manuals berücksichtigt daher konventionelle etablierte Zusatzverfahren, methodische Neuerungen und Auswertemöglichkeiten sowie unterschiedliche spezielle Indikationsstellungen bei zunehmend wichtigen Patientengruppen:

a. Die transösophageale Echokardiographie

b. Die Stressechokardiographie

c. Die Kontrastechokardiographie

d. Die transthorakale und transösophageale 3D-Echokardiographie

e. Das Deformationsimaging

f. Die Echokardiographie bei angeborenen Herzfehlern im Erwachsenenalter

g. Die Echokardiographie bei und nach kardiochirurgischen Operationen

h. Die Echokardiographie beim Monitoring von Interventionen bei strukturellen Herzerkrankungen

i. Die Echokardiographie bei temporären und permanenten kardialen Unterstützungssystemen

j. Vorsichtsmaßnahmen bei der Echokardiographie hochinfektiöser Patienten in Pandemien

Während die transthorakale Echokardiographie (TTE) universell einsetzbar, schnell durchführbar und preiswert ist und nahezu keine Kontraindikationen aufweist, sind transösophageale Echokardiographie (TEE), Kontrast- und Stressechokardiographie semiinvasive Untersuchungen, die in der Regel einer Aufklärung des Patienten bedürfen und einer strengen Indikationsstellung unter Berücksichtigung der Kontraindikationen unterliegen. Trotzdem sind sie nahezu bei allen kardialen Erkrankungen zur genauen Diagnosestellung in speziellen Fällen notwendig. Besonders für Zusatzverfahren und semiinvasive Untersuchungen der Echokardiographie sind eine Ausstattung mit qualitativ hochwertigen Geräten und eine qualifizierte Dokumentationsqualität notwendig, die nur durch eine entsprechende Ausbildung und die damit verbundene Untersucherqualität zu gewährleisten sind [11, 34]. Bei allen im Folgenden angesprochenen Themen gelten grundsätzlich vor jeder Zusatzuntersuchung und spezieller Auswer-

Das zweite Manual zur Indikation und Durchführung der Echokardiographie bezieht sich auf spezifische Anwendungen der Echokardiographie und besondere Fragestellungen bei speziellen Patientengruppen. Dabei stehen v. a. praktische Aspekte im Vordergrund. Methodisch etabliert sind die transösophageale Echokardiographie, die Stressechokardiographie und die Kontrastechokardiographie. Bei nahezu allen echokardiographischen Untersuchungen spielen aktuell 3-DEchokardiographie und Deformationsbildgebung eine Rolle. Das gesamte Spektrum der echokardiographischen Möglichkeiten wird derzeit in Notfall- und Intensivmedizin, bei der Überwachung und Führung von Katheterinterventionen, bei strukturellen Herzerkrankungen, bei herzchirurgischen Operationen, bei der Nachsorge von kardialen Unterstützungssystemen, bei kongenitalen Vitien im Erwachsenenalter und bei der Versorgung von hochinfektiösen Patienten in Pandemiezeiten angewandt. Die diagnostischen Fortschritte der konventionellen und modernen echokardiographischen Anwendungen stehen im Fokus dieses Manuals. Die 3-DEchokardiographie zur Charakterisierung der kardialen Morphologie und die Deformationsbildgebung zur Objektivierung der kardialen Funktion sind bei vielen Indikationen im klinischen Alltag etabliert. Die Stressechokardiographie zur Ischämie-, Vitalitäts- und Vitiendiagnostik, die Bestimmung der koronaren Flussreserve und die Kontrastechokardiographie bei der linksventrikulären Wandbewegungsanalyse und kardialen Tumordetektion finden zunehmend klinische Anwendung. Wie für die konventionelle Echokardiographie im ersten Manual der Echokardiographie 2009 beschrieben, erfordert der Einsatz moderner echokardiographischer Verfahren die standardisierte Dokumentation und Akquisition bestimmter Bildsequenzen bei optimierter Geräteeinstellung, da korrekte und reproduzierbare Auswertungen nur bei guter Bildqualität möglich sind.

\section{Schlüsselwörter}

Transösophageale Echokardiographie · Stress-Echokardiographie · Kontrast-Echokardiographie · Deformations-Bildgebung · Echokardiographisches Monitoring

tung die Regeln einer qualifizierten TTE mit der Durchführung des „standardisierten Untersuchungsgangs", um transparent, reproduzierbar und objektiv alle wichtigen und notwendigen Befunde mit den daraus resultierenden Diagnosen zu erheben bzw. auszuschließen. Im Folgenden wird auch bei Zusatzuntersuchung auf eine Standardisierung und die dazugehörige Minimalanforderung an die echokardiographische Dokumentation Bezug genommen.

\section{Die transösophageale Echokardiographie (TEE)}

Die TEE ermöglicht nahezu immer eine echokardiographische Untersuchung mit guter Bildqualität. Aufgrund der sehr geringen Distanz zwischen dem Transducer in Ösophagus und Herzklappen, Vorhöfen und Aorta ascendens sowie descendens kann routinemäßig mit höheren Schallfrequenzen $(5-8 \mathrm{MHz}$ ) und im fundamentalen Modus untersucht werden, wodurch eine höhere räumliche Auflösung im Vergleich zur TTE erlangt werden kann. Die Indikationen (s. - Tab. 1) betreffen daher primär die dem Ösophagus schallkopfnahen kardialen Strukturen. Eine TEE kann jedoch grundsätzlich bei allen echokardiographischen Fragestellungen sinnvoll sein, bei denen die TTE-Bildqualität nicht ausreichend ist. Spezielle Fragestellungen, wie z. B. der Ausschluss von Thromben im linken Vorhof und Herzohr, der Verdacht auf eine Endokarditis - speziell bei Klappenprothesen - und die Detektion oder der Ausschluss einer thorakalen Aortendissektion, rechtfertigen auch direkt eine TEE zur Diagnosesicherung. Die TEE kann in der Regel bei wachen Patienten unter lokaler Rachenanästhesie durchgeführt werden $[24,25,38]$. Eine Sedierung mit Midazolam oder alternativ Propofol i.v. kann individuell vorgenommen werden. Wichtige Vorsichtsmaßnahmen (mindestens $4 \mathrm{~h}$ keine feste Nahrung vor der TEE, intravenöser Zugang für evtl. Medikamentengaben, Puls- und Blutdrucküberwachung, idealerweise kontinuierliche Sauerstoffsättigungsmessung, Bereitstellung von Intubationsmöglichkeiten, Absaugevorrichtungen und notwendiges Equipment für eine Reanimation) sind einzuhalten. Die 
Tab. 1 Indikationen, Zielstrukturen und Befunde bei der TEE

Die TEE hat im Vergleich zur TTE eine klar be-

Die Analyse des linken Vorhofohres (LAA) im
Indikationsstellung und Begründung

Infektiöse Endokarditis legte höhere diagnostische Genauigkeit. Bei begründetem Verdacht auf Endokarditis und negativer TTE ist daher eine TEE erforderlich

\section{Intrakavitäre Raumforderungen}

Linksatriale Thromben können im TTE grundsätzlich nicht sicher detektiert werden und erfordern daher eine TEE TEE ist komplex
Zielstruktur

Endokarditisch bedingte Läsionen

Abszedierungen

Pseudoaneurysmen

Fisteln, Vegetationen an Nativklappen bzw.

an Fremdmaterial

Paraprothetische Leckagen

Thromben

Ventrikulär

Atrial

Im linken Vorhofohr (LAA)

Tumoren

Myxome (am häufigsten)

Benigne und maligne Tumoren

Metastasen

\section{Herzklappenerkrankungen}

Die TEE weist im Vergleich zur TTE eine höhere räumliche Auflösung auf und ist daher primär geeignet zur Analyse von Klappenmorphologien die TEE in der Analyse der Klappenfunktion

\section{Mitralklappeninsuffizienz}

Mitralklappenstenose

Aortenklappeninsuffizienz

Aortenklappenstenose

Trikuspidalklappeninsuffizienz

Trikuspidalklappenstenose
Die TTE ist bei guter Schallbarkeit besser als

Befund

Größe und Beweglichkeit von Vegetationen Ausdehnung und Ausspülung von Abszedierungen

Funktionelle Relevanz von Pseudoaneurysmen, Fisteln und Leckagen

Größe und Beweglichkeit von Thromben und Tumoren

LAA-Analyse

Darstellung der Morphologie einschließlich

aller Seitenlobi

Dokumentation von Sludge und Spontankontrast

Messung der LAA-Ein- und Ausflussgeschwindigkeiten

\section{Klärung der Genese des Vitiums}

Bei Insuffizienzen:

Dokumentation der Morphologie und Lokalisation der Läsion - speziell im Hinblick auf die Option einer Klappenrekonstruktion Einstufung des Schweregrades der Insuffizienz (eingeschränkte Beurteilbarkeit bei Sedierung) Bei Stenosen:

Dokumentation der Morphologie und Lokalisation der Läsion - speziell die Klappenöffnungsfläche

Einstufung des Schweregrades der Stenose

\section{Klappenprothesen}

Die TEE hat im Vergleich zur TTE eine klar belegte höhere diagnostische Genauigkeit in der Diagnostik der Prothesendysfunktion

\section{Korrelate einer Prothesenendokarditis}

Prothesenthrombose

Pannus

Paraprothetische Leckagen

Prothesendysfunktion
Morphologie der Prothese

Dokumentation der Morphologie (Größe und Beweglichkeit) und Lokalisation von zusätzlichen Strukturen

Funktion der Prothese

Einstufung des Schweregrades von Insuffizienzen und Stenosen im Falle von Degenerationen (Bioprothesen) oder Komplikationen (Kunstprothesen)

Ausdehnung und Größe von Ektasien/
Aneurysmen Entry und Reentry der Dissektionslamelle Lokalisation von Rupturen Ausdehnung und Beschaffenheit von Plaques kalen Aorta wie die Computertomographie Die TEE hat jedoch einen "blind spot" in der Aorta ascendens in Höhe der trachealen Bifurkation

\section{Intrakardiale Kommunikationsdefekte}

Die definitive Klärung der Anatomie sowie deren funktioneller Relevanz ist vor interventionellem Verschluss der Shunts notwendig

Aneurysmen
Dissektionen
Rupturen
Plaqueformationen und murale Hämatome

Offenes Foramen ovale (PFO)

Vorhofseptumdefekt

Septum-primum-Defekt

Septum-secundum-Defekt

Oberer und unterer Sinus-venosus-Defekt

Koronarsinusdefekt

Ventrikelseptumdefekt

Perimembranöser Defekt

Muskulärer Defekt

Dokumentation des Operationsergebnisses herzchirurgischer Eingriffe - speziell Klappenrekonstruktionen

Unterscheidung zwischen kongenitalen und
erworbenen, z. B. postinfarziellen VSD
Größe und Beschaffenheit der Defekte
Nachweis durch 2-D- und 3-D-Morphologie,
farbkodierte Dopplerechokardiographie und
Rechtsherzkontrastgabe
Eindeutige Klärung der Randstrukturen der
Defekte
Detektion sonstiger Anomalien - insbesonde-
re fehleinmündender Lungenvenen
Rekonstruktionen von Herzklappen (Aorten-
und Mitralklappe)
Beurteilung von Koaptation und Leckagen
Beurteilung der linksventrikulären Funktion
Prothetischer Klappenersatz
Beurteilung von paravalvulären Leckagen
Aortenwurzel-/Aorta ascendens- und Aorten-
bogenoperationen
Beurteilung von Nahtinsuffizienzen

postinfarziellen VSD Nachweis durch 2-D- und 3-D-Morphologie, farbkodierte Dopplerechokardiographie und Rechtsherzkontrastgabe

Defekte

Detektion sonstiger Anomalien - insbesonde-

Rekonstruktionen von Herzklappen (Aortenund Mitralklappe)

Beurteilung der linksventrikularen Funktion Prothetischer Klappenersatz

Aortenwurzel-/Aorta ascendens- und Aorte Beurteilung von Nahtinsuffizienzen 
Tab. 1 (Fortsetzung)

Indikationsstellung und Begründung

Monitoring von Katheterinterventionen bei

strukturellen Herzerkrankungen
Zielstruktur

Verschluss intrakardialer Kommunikationsde-

fekte

Ductus-Verschluss

LAA-Verschluss

Mitral- und Trikuspidalklappeninterventio-

nen, u. a. Clipping der AV-Klappen

Verschluss paravalvulärer Leckagen nach

Klappenersatz
Befund

Eindeutige Dokumentation der zu behan-

delnden Struktur

Darstellung aller katheterinterventionellen

Maßnahmen

Dokumentation des Device-Deployments

Dokumentation postprozeduraler Verände-

rungen
TEE-Sonden sind vor Gebrauch regelmäßig auf ihre Intaktheit (Oberflächenkontrolle) zu überprüfen. Weiterhin müssen die Sonden und die Geräte vor ihrem Einsatz bzw. nach jedem Gebrauch vorschriftsmäßig desinfiziert werden. Bei Einsatz von latexfreien Überzügen sollte deren Füllung mit endoskopischem Gel ohne Einschluss von Luftpartikeln erfolgen. Probleme beim Einführen der TEE-Sonde im Sinne eines Widerstandes im oberen Rachenraum oder Ösophagus beim Vorschub der proximalen TEE z.B. in die Recessus piriformes oder klare Schmerzen müssen zum Abbruch der TEE-Sondierung führen. Sehr selten (ca. 1-3/10.000) kommt es zur Verletzung des Ösophagus mit Ruptur, Blutung und/oder konsekutiver Mediastinitis, deren Symptome mit Verzögerung auftreten können. Kontraindikationen zur TEE sind Pathologien im oberen Gastrointestinaltrakt, wie z.B. Tumoren, Divertikel und Strikturen der Speiseröhre. Relative Kontraindikationen sind eine schwere Thrombopenie und eine schwere pulmonale Hypertonie. Eine Endokarditisprophylaxe ist nach aktuellen Empfehlungen nicht erforderlich.

Der TEE-Untersuchungsgang kann nach unterschiedlichen Strategien durchgeführt werden. Beim wachen oder sedierten Patienten handelt es sich oft aus Gründen des fehlenden Komforts und der damit verbundenen Einschränkung der Toleranz des Patienten um eine limitierte Untersuchung, bei der z.B. die oft beschwerliche Untersuchung von gastral ausgelassen wird oder nur eine zielgerichtete, relativ kurze Untersuchung durchgeführt wird, wie z.B. nur die Darstellung des linken Vorhofes und des linken Vorhofohres zum Ausschluss von Thromben vor Kardioversion. Ähnlich bestehen im Notfall zeitliche Limitationen, wenn z. B. durch hohe Körpertemperatu- ren nur eine begrenzte Untersuchungszeit zur Verfügung steht, bevor die TEE-Sonde wegen drohender Überhitzung ihre Funktion einstellt. Dementsprechend konzentrieren sich fokussierte Untersuchungen auf die Beantwortung der konkreten Fragestellung, wie z.B. die Suche nach endokarditisch bedingten Läsionen bei Infektkonstellationen. Toleriert der Patient die Untersuchung ausreichend oder findet sie in Narkose statt (Intensivstation, Katheterlabor, Operationsraum), kann eine komplette, systematische Untersuchung aller Herzstrukturen nach anatomischen Aspekten durchgeführt werden, die dann in der Regel distal mit dem transgastralen Schallfenster beginnt und dann die ösophagealen Schallfenster im Rückzug durchläuft. Die typischen diagnostischen Schallkopfpositionen der TEE sind weniger scharf definiert als bei der TTE, da das transösophageale „Fenster" kontinuierlich ist. Schematisch werden eine obere, mittlere und tiefe transösophageale und eine transgastrale Schallkopfposition unterschieden. Die derzeitigen etablierten modernen Sonden erlauben die mechanische Steuerung der Sondenspitze (mediallateral und anterior-posterior) sowie die Rotation der Ultraschallebene um $180^{\circ}$. Hiermit werden im Wesentlichen an transthorakale Standardschnitte angelehnte Schnittebenen eingestellt, wobei einige Ebenen wie die „bicavale“ Schnittebene der Vorhöfe, die Darstellung des linken Herzohrs oder der Aorta descendens keine Entsprechung im TTE-Standardprogramm haben.

Seit etwa 3 Jahrzehnten gehört die intraoperative TEE bei herzchirurgischen Klappeneingriffen zum diagnostischen Standard, der insbesondere nach Abgang von der Herz-Lungen-Maschine erlaubt, das funktionelle Ergebnis klappenchirurgischer Eingriffe zu überprüfen.
In den letzten Jahren haben sich bei Katheterinterventionen (u.a. Verschluss von intrakardialen Kommunikationsdefekten, Verschluss des linken Vorhofohres, Herzklappeninterventionen, Verschluss paraprothetischer Leckagen, Biopsien intrakardialer Tumoren) die Führung der Intervention und das Monitoring der Ergebnisse durch die simultane TEE durchgesetzt. Die Ausbildung zur TEE sollte in der Regel nach Erlernen des TTE-Untersuchungsganges erfolgen. Der Einsatz von TEE-Simulatoren in der Einarbeitung hat sich als hilfreich erwiesen.

In der $\bullet$ Tab. 1 sind Indikationen, Zielstrukturen und Befunde bei einer TEE zu bestimmten Fragestellungen und Krankheitsentitäten zusammengefasst.

\section{Die Stressechokardiographie}

Stressechokardiographie zur Ischämie- und Vitalitätsdiagnostik

Die Indikation zur Stressechokardiographie ist in diesem Zusammenhang gegeben bei

- Verdacht auf koronare Herzerkrankung (KHK) [36, 84, 100, 104],

- Verlaufskontrollen bei bekannter KHK [36, 84, 100, 104],

- Beurteilungen der Myokardvitalität zur Klärung der Notwendigkeit einer Revaskularisierung [36, 84, 100, 104] und

- erweiterten Beurteilungen der kardialen Funktion und Hämodynamik in Gegenwart von Vitien $[9,12,18,26$, $43,50,52,54-56,58,65,67,87,88,96$, $108]$.

Die Stressechokardiographie konkurriert mit anderen Belastungsuntersuchungen mit gleichzeitiger Bildgebung wie die Kernspintomographie und Szintigraphie. 
Obwohl die Stressechokardiographie ubiquitär verfügbar sein sollte, sind genaue Angaben zur Häufigkeit ihrer Durchführung aktuell in Deutschland nicht verfügbar. Zur Auswahl stehen folgende

Stressmodalitäten (s. ๑ Abb. 1):

- dynamische oder physikalische Stressechokardiographie mittels Fahrradergometrie oder Laufbandbelastung,

- pharmakologische Stressechokardiographie mit inotropen (Dobutamin) oder vasodilatierenden Substanzen (Adenosin, Dipyridamol).

Zu den Voraussetzungen zur Durchführung einer Stressechokardiographie bei Verdacht auf KHK gehören

- grundsätzlich eine TTE vor einer Stressechokardiographie, um kardiale Pathologien zu objektivieren bzw. auszuschließen,

- die schriftliche Aufklärung des Patienten, insbesondere erforderlich bei pharmakologischem Stress und bei Verwendung von Ultraschallkontrastverstärkern (Echokontrast),

- Pausierung von $\beta$-Blockern, damit die Zielherzfrequenz oder eine signifikante Vasodilatation erreicht werden kann. Die Pausierung hängt von dem jeweiligen $\beta$-Blocker ab ( 1 bis 3 Tage vor der Untersuchung: z. B. längere Pause bei Bisoprolol und Sotalol, kürzere Pause bei Metoprolol); die übrige Medikation kann in der Regel eingenommen werden. Bei der dynamischen Stressechokardiographie ist eine Nüchternheit nicht zwingend erforderlich,

- bei Adenosin-Stress zudem Pausierung von Koffein, Nikotin, Schokolade, Energy-Drinks sowie Theophyllin-haltigen Medikamenten am Vortag der Untersuchung und am Untersuchungstag.

Kontraindikationen für alle Stressmethoden sind sorgfältig vor der Untersuchung zu klären bzw. bei der Untersuchung zu berücksichtigen.

- Absolute Kontraindikationen sind Notfallsituationen wie u. a. das akute Koronarsyndrom, der Myokardinfarkt, die Lungenembolie und die Aortendissektion.

- Relative Kontraindikationen bestehen bei symptomatischen Patienten in Ruhe (z. B. bei hochgradiger Aortenklappenstenose [AS], malignen Arrhythmien und hypertensiven Entgleisungen) sowie insbesondere im Fall von Dobutamin-Stressechokardiographien bei einer signifikanten Ausflusstraktobstruktion des linken Ventrikels.

- Kontraindikationen für die Vasodilatator-Stressechokardiographie sind atrioventrikuläre (AV) Leitungsverzögerungen $\mathrm{II}^{\circ}$ und $\mathrm{II}^{\circ}$ bei fehlender Schrittmacherimplantation, symptomatische Hypotonien und Asthma bronchiale.

- Unter Berücksichtigung der Kontraindikation sind die Risiken aller Stressechokardiographie-Modalitäten niedrig. Dabei wird in der Literatur die Dobutamin-Stressechokardiographie mit der höchsten Komplikationsrate für lebensbedrohliche Ereignisse angegeben.

Bei der Durchführung der Stressechokardiographie ist das Ziel das Erreichen der Ausbelastung bzw. die Detektion eines eindeutigen pathologischen Befundes.

- Bei der dynamischen Stressechokardiographie sollte somit die maximale, nach Alter und Geschlecht berechnete Herzfrequenz erreicht werden, da pathologische Veränderungen sich oft nicht vor der Ausbelastung zeigen und damit die Aussagekraft der Untersuchung eingeschränkt ist.

- Falls vor dem Erreichen der Zielfrequenz linksventrikuläre (LV) Wandbewegungsstörungen oder signifikante EKG-Veränderungen dokumentiert werden, kann die Untersuchung aufgrund eines positiven Ergebnisses abgebrochen werden.

- Symptome wie Angina pectoris, Präsynkopen, Synkopen, ein symptomatischer Blutdruckabfall sowie -anstieg, neu aufgetretenes Vorhofflimmern und/oder maligne ventrikuläre Arrhythmien sollten zum Abbruch der Untersuchung führen.

- Weiterhin gelten ein Anstieg von E/e' > 15, ein Abfall der TAPSE (,tricuspid anular plane systolic excursion") $>4 \mathrm{~mm}$, ein rechtsventrikulärer systemische Druck (RVSP) $>60 \mathrm{~mm} \mathrm{Hg}$ und/oder eine mutmaßliche ischämi- sche Mitralklappeninsuffizienz als pathologische Befunde einer dynamischen Stressechokardiographie, die in der Regel durch die begleitende Symptomatik zum Abbruch führen. Die Ursachen dieser Befunde müssen allerdings nicht immer ischämischer Genese sein.

- Zusätzliche Abbruchkriterien sind muskuläre Erschöpfung, eine hypertensive Entgleisung oder sonstige Beschwerden, die eine weitere Belastung verhindern.

- Bei der Dobutamin-Stressechokardiographie ist ein Blutdruckabfall durch eine pharmakologisch induzierte Ausflussbahnobstruktion des linken Ventrikels infolge der Abnahme des enddiastolischen Volumens in Kombination mit einer erhöhten Inotropie ebenfalls ein Abbruchkriterium.

Die Dokumentationsprotokolle für Stressechokardiographien sollten bei der Ischämiediagnostik folgende Aspekte beinhalten.

- Neben der kontinuierlichen 1-KanalEKG-Ableitung ist die zusätzliche Dokumentation des EKGs während jeder Belastungsstufe erforderlich. Bei der dynamischen und DobutaminStressechokardiographie ist ein 12Kanal-EKG sinnvoll.

- Eine intermittierende nichtinvasive Blutdruckmessung sollte bei jeder Belastungsstufe durchgeführt werden.

- Nach den aktuellen Empfehlungen gehören zur Basisdokumentation die parasternale lange und kurze Achse sowie die apikale lange Achse und der 2- und 4-Kammer-Blick. Aus praktischen Gründen empfiehlt es sich, 2 bis 3 Herzzyklen aufzunehmen, um nach der Untersuchung den Zyklus mit der besten Bildqualität auswählen zu können.

- Zur Beurteilung der diastolischen Funktion, der Klappenfunktionen oder der Rechtsherzfunktionen sind an den Ultraschallgeräten zusätzliche Dokumentationseinstellungen von Dopplerspektren oder farbkodierten Dopplersequenzen in den Stressprotokollen notwendig (Bestimmung der TAPSE, Gradienten über den Klappen, 


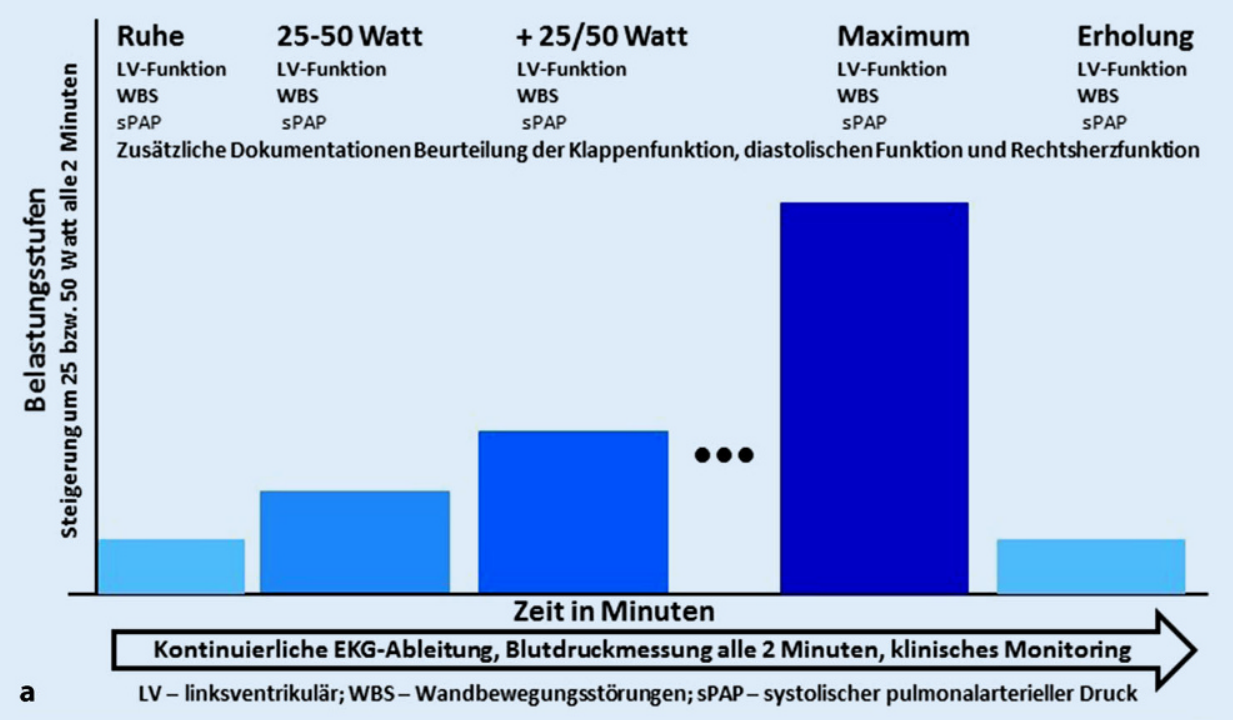

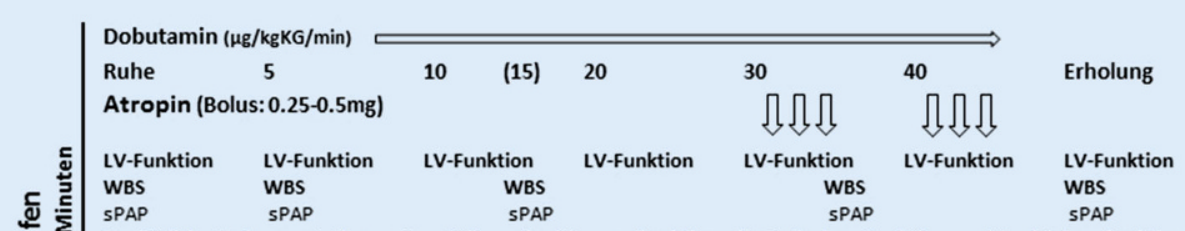

营

Zusätzliche Dokumentationen Beurteilung der Klappenfunktion, diastolischen Funktion und Rechtsherffunktion

b IV - linksventrikulär; WBS- Wandbewegungsstörungen; SPAP - systolischer pulmonalarterieller Druck

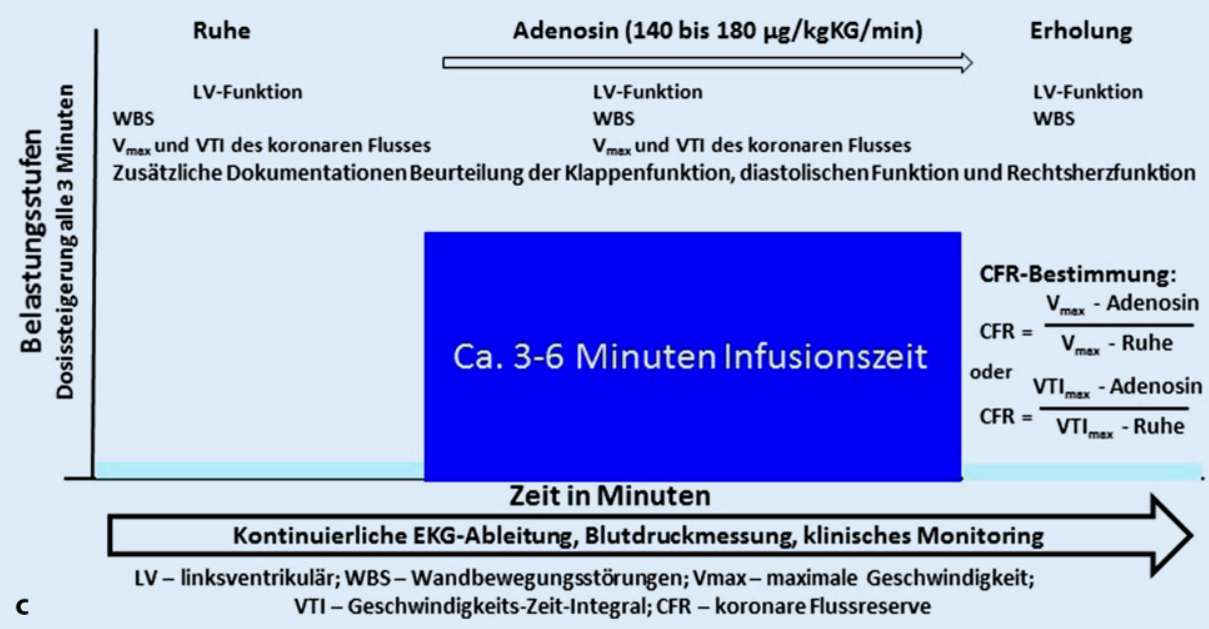

Abb. $1<$ Schematische Darstellung der Stressprotokolle für eine dynamische Stressechokardiographie (a), eine Dobutamin-Stressechokardiographie (b) und eine Adenosin-Stressechokardiographie (c) 
Akzelerations- und Dezelerationszeiten, $E / e^{\prime}$ [septal] etc.).

- Eine simultane triplane Registrierung der apikalen Schnittebenen ist aufgrund von Zeitersparnis sowie bei Rhythmusstörungen hilfreich.

- Bei zusätzlicher Speckle-TrackingAnalyse ist auf eine Bildrate zwischen 40 und 80/s zu achten.

- Bei Nichtbeurteilbarkeit von 2 oder mehr topographisch zusammenhängenden Wandsegmenten des linken Ventrikels wird die Verwendung von Linksherzkontrastverstärkern bei der Stressechokardiographie empfohlen.

- Während jeder Belastungsstufe sollten die jeweiligen Darstellungsebenen zur Identifikation von stressinduzierten Wandbewegungsstörungen im Vergleich zu Ruhe zeitgleich beurteilt werden können. Die Bildsequenzen jeder Belastungsstufe sollten in digitaler Form gespeichert werden, um nach der Untersuchung mittels geeigneter Software neu aufgetretene Wandbewegungsstörungen identifizieren zu können.

Die Auswertung der Bilddaten erfolgt im Anschluss an die komplette Datenakquirierung durch Vergleich der Bildsequenzen.

- In der Regel wird überwiegend eine rein visuell vergleichende, subjektive Analyse mittels sog. „Quadscreen“Darstellung am Ende der Untersuchung vorgenommen.

- Die regionale Wandbewegung des linken Ventrikels wird über eine genaue Endokarderkennung mit dem sog. 16-, 17- oder 18-Segment-Modell beurteilt.

Ein positiver Befund der Stressechokardiographie liegt vor, wenn mindestens in 2 benachbarten Segmenten neue Wandbewegungsstörungen auftreten, die durch das Hauptkriterium der Wanddickenzunahme in der jeweiligen myokardialen Wand charakterisiert und durch das Nebenkriterium der Einwärtsbewegung des Endokards charakterisiert sind.

- Eine alleinige Einwärtsbewegung ohne Wanddickenzunahme ist kein Nachweis für eine normale Myokardfunktion, da passive Einwärtsbewegungen durch aktive Kontraktion benachbar- ter gesunder Segmente möglich sind ("tethering").

- Die Einteilung der Wandbewegungsanalyse unterscheidet die Normokinesie (Wanddickenzunahme $>50 \%$ ), die Hypokinesie (Wanddickenzunahme $<40 \%$ ), die Akinesie (Wanddickenzunahme $<10 \%$ ), die Dyskinesie (systolische Auswärtsbewegung) und die Aneurysmaformation (diastolische Deformation). Akinetische oder dyskinetische Areale mit Wanddicken $<6 \mathrm{~mm}$ und vermehrter Textur entsprechen in der Regel Narben.

- Pathologische Befunde sind nicht immer auf eine koronare Makroangiopathie zurückzuführen. Sie können auch bei mikrovaskulären Erkrankungen, hypertensiven Entgleisungen oder Kardiomyopathien auftreten.

- Die verschiedenen Grade der Wandbewegungsstörungen in Ruhe und unter Belastung werden zur Berechnung des Wall Motion Score Index (WMSI) verwendet, bei welchem die Summe des Scores durch die Summe der beurteilten Wandsegmente geteilt wird (normal = 1, pathologisch > 1). Basierend auf diesem Score können therapeutische Konsequenzen getroffen werden und sind prognostische Aussagen möglich.

- Als weiterer Index kann auch der „Echokardiographie-Score" verwendet werden. Auf höchster Belastungsstufe werden 0 Punkte für normal, 1 Punkt für hypokinetisch, 2 Punkte für akinetisch, 3 Punkte für dyskinetisch und 4 Punkte für aneurysmatisch vergeben und die Summe gebildet (Umrechnung WMSI = 1 + Echokardiographie-Score/ Anzahl beurteilte Wandsegmente).

- Eine sog. postsystolische Kontraktion nach Aortenklappenschluss ist ebenfalls ein mögliches Ischämiezeichen des Myokards. Sie ist in der Regel aufgrund ihrer zeitlichen Zuordnung innerhalb des Herzzyklus nur zuverlässig bei Verwendung eines sog. anatomischen M-Modes zu erkennen.

- Der Ablauf einer dynamischen Stressechokardiographie ist in $\mathbf{0}$ Abb. 1a skizziert. Neben der in Deutschland üblichen dynamischen Fahrrad-Stressechokardiographie wird in anderen Ländern auch Laufbandbelastung durchgeführt, bei denen bei den Patienten die Ultraschallsequenzen nur direkt vor und nach der Belastung akquiriert werden. Eine Laufbandbelastung wird daher von der DGK nicht empfohlen. Ein Pharmakon ist als Stressor indiziert, wenn eine dynamische Stressechokardiographie nicht durchgeführt werden bzw. bei dieser nicht eine submaximale Belastung erreicht werden kann. Zur Ischämiediagnostik werden bei Dobutamin Dosierungen von 10-40 $\mu \mathrm{g} / \mathrm{kg}$ Körpergewicht $(\mathrm{KG}) / \mathrm{min}$ in $10-\mu \mathrm{g}$-Schritten verwendet. Dobutamin sollte vorzugsweise mittels Infusomat oder Perfusor in Verdünnung $(50 \mathrm{ml}$ Dobutamin plus $200 \mathrm{ml}$ 0,9\% NaCl-Lösung) appliziert werden, da durch die höheren Flussraten die Wirkung schneller eintritt. Bei geringen Herzfrequenzanstiegen können Bolusapplikationen von Atropin ab $30 \mu \mathrm{g} / \mathrm{kgKG} / \mathrm{min}$ zusätzlich gegeben werden. Die maximale Atropin-Dosis von 1-2 mg/Untersuchung sollte nicht überschritten werden. Der Ablauf der Dobutamin-Stressechokardiographie ist in - Abb. 1b skizziert. Die Adenosin-Stressechokardiographie wird zur Bestimmung der koronaren Flussgeschwindigkeitsreserve (CFR) durch Messung der koronaren Blutflussgeschwindigkeiten in distalen Abschnitten der Koronargefäße oder durch Analyse der Perfusion mittels der myokardialen Kontrastechokardiographie verwendet. Bei dieser Stressmodalität wird eine konstante Infusionsrate von 140-180 $\mu \mathrm{g} / \mathrm{kgKG} / \mathrm{min}$ über 3-6 min empfohlen. Der Ablauf der Adenosin-Stressechokardiographie ist in

- Abb. 1c skizziert. Das in anderen Ländern häufig verwendete Dipyridamol ist in Deutschland für die Stressechokardiographie nicht verfügbar. Die Bestimmung der CFR erfolgt über den Vergleich der maximalen Vasodilatation unter Adenosin-Infusion gegenüber der Ruhemessung. Der CFR-Wert wird über die Ratio der jeweiligen diastolischen Maximalgeschwindigkeiten oder der diastolischen GeschwindigkeitsZeit-Integrale berechnet, wobei eine Ratio $>3,3$ als normal, von 2,7 bis 3,3 als minimal reduziert, von 2,0 bis 2,7 als leicht reduziert, von 1,75 bis 1,99 
als mäßig reduziert mit Hinweisen auf signifikante Ischämie sowie von 1,0 bis 1,74 als hochgradig reduziert mit Hinweisen auf eine schwere Ischämie gilt.

Ein CFR-Wert $<2,0$ ist eng assoziiert mit einer $F F R<0,8$. Die nichtinvasiv gemessene CFR kann daher zur Verlaufskontrolle von intermediären Koronarstenosen v. a. im Bereich der LAD verwendet werden.

Die Kontrastechokardiographie mit lungengängigen Ultraschallverstärkern für die myokardiale Kontrastechokardiographie (MCE) ermöglicht neben der alleinigen Wandbewegungsanalyse zusätzlich die Detektion von Perfusionsdefekten, die Ischämie-induzierten Wandbewegungsstörungen zeitlich vorausgehen und somit die Sensitivität des Ischämienachweises erhöhen. Empfohlen werden die Abgabe eines Impulses mit hoher Sendeleistung („flash") sowie die Registrierung der nachfolgenden Wiederauffüllung der Mikrozirkulation („replenishment") mit Ultraschallkontrastverstärker mit gleichzeitiger Analyse von Wandbewegung und Perfusion. Es handelt sich jedoch dabei um eine Offlabel-Anwendung, die eine kontrastspezifische Software voraussetzt (MultipulsTechnik mit sehr niedriger Sendeleistung) sowie - wie bei allen Perfusionsverfahren in der klinischen Anwendung - eine erweiterte Schulung des Anwenders. Für die Wiederanflutung gelten Zeitintervalle von $4-5 s$ in Ruhe und $<2 s$ unter Vasodilatation. Die MCE kann hilfreich sein bei Patienten mit bereits in Ruhe bestehenden Wandbewegungsstörungen wie bei Schenkelbockbildern, ventrikulärer Stimulation und nichttransmuralen Narben, um eine Ischämie unter Belastung auch unabhängig von der Wandbewegung zu erkennen. Die Methode kann auch bei Mehrgefäßerkrankungen eingesetzt werden, da die Verringerung der absoluten Anflutungszeit als Ischämiekriterium dienen kann. Dadurch ist ein Vergleich zu nichtischämischen Myokardabschnitten nicht zwingend erforderlich. Allerdings beinhaltet die Stressechokardiographie mittels MCE einen höheren Aufwand aufgrund der längeren Vorbereitung, des höheren Gerätebedarfs und der höheren Kosten.
Bei bestehender Wandbewegungsstörung in Ruhe kann durch eine dynamische oder Dobutamin-Stressechokardiographie auch die Vitalität des Myokards analysiert werden. Schon auf niedrigen Belastungsstufen zeigt sich vitales Myokard bedingt durch den positiv inotropen Effekt durch eine Zunahme der Kontraktilität (kontraktile Reserve) durch eine Wanddickenzunahme. Eine Myokardischämie kann auch durch eine diastolische Stressechokardiographie detektiert werden, wobei der Surrogatparameter E/e' des LV-enddiastolischen Drucks (LVEDP) analysiert wird. Ein LVEDP-Anstieg zeigt sich durch eine pathologische Erhöhung von E/e' über 15 unter Belastung, wobei in der Regel der septale $e^{\prime}$-Wert bestimmt wird. Bei höheren Herzfrequenzen über 100-120/min ist die Analyse der diastolischen Funktion jedoch aufgrund der Fusion von E- und A-Welle limitiert. Die Bestimmung von E- und A-Wellen-Geschwindigkeiten kann jedoch in der Nachbelastungsphase bis zu $5 \mathrm{~min}$ nach Belastungsende erfolgen. Bei Verdacht auf eine rechtsventrikuläre Ischämie kann die TAPSE als zusätzlicher Parameter verwendet werden. Eine Abnahme der TAPSE $>4 \mathrm{~mm}$ deutet auf eine relevante rechtsventrikuläre Ischämie hin. Eine unauffällige Stressechokardiographie bedeutet eine gute Prognose, da die Patienten in dieser Gruppe mit einer Mortalität von 0,4-0,9\%/Jahr dasselbe Risiko wie altersadjustierte Gesunde haben. Eine unauffällige Stressechokardiographie ist mit einer im Vergleich zu altersadjustierten Gesunden nicht erhöhten kardiovaskulären Mortalität von $0,4-0,9 \% / J a h r$ assoziiert, sofern eine ausreichende Beurteilbarkeit aller Wandsegmente besteht und der Untersucher nach Empfehlungen der DGK bzw. der EACVI und ASE ausreichend geschult ist.

Moderne Modalitäten wie die 3-DEchokardiographie und die Strain-Analysen finden neben der Kontrastechokardiographie zunehmend Anwendung in Klinik und Praxis. Allerdings bestehen wichtige Limitationen, wie z. B. die begrenzte zeitliche Auflösung der 3-D-Echokardiographie und die hohen Anforderungen an die Bildqualität beim Speckle-Tracking.

Der Befund einer Stressechokardiographie sollte die Stressmodalität, die maximale Belastungsstufe, die Schallbarkeit des Patienten, den Blutdruck- und EKG-Verlauf und mögliche Abbruchkriterien sowie abschließend die Beurteilung des Testergebnisses beinhalten.

\section{Stressechokardiographie bei Vitien}

Eine Stressechokardiographie bei Patienten mit nativen Herzklappenerkrankungen sollte generell bei sog. asymptomatischen relevanten Vitien in Betracht gezogen werden, da durch den Belastungstest Symptome objektiviert werden können und eine Adaptation an ein zu geringes Belastungsniveau festgestellt werden kann. Zudem kann bei Vitien mit mittelgradig eingestuftem Schweregrad durch eine Belastung eine Behandlungsnotwendigkeit durch provozierte Beschwerden und kardiale Veränderungen dokumentiert werden. Als etabliert gelten die dynamische Stressechokardiographie bei der Mitralklappenstenose (MS) unklaren Schwergrades sowie die "Low-Dose"-Dobutamin-Stressechokardiographie bei der "Low Flow (LF) - Low Gradient (LG)" AS mit reduzierter LV Ejektionsfraktion (LVEF) $[26,88]$. Das zugrunde liegende Ziel einer Belastungsuntersuchung ist es, die Symptomatologie und damit das individuelle Risiko der Patienten möglichst genau einzuschätzen und basierend auf den Befunden den optimalen Zeitpunkt für eine interventionelle oder operative Therapie festzulegen $[5,57,58,79]$. Determinanten, die durch eine Belastungsuntersuchung aufgedeckt werden können und für eine frühzeitige interventionelle/operative Therapie sprechen, sind neu auftretende klinische Beschwerden, ein Abfall des Blutdrucks, ein relevanter Anstieg der Gradienten bei stenosierenden Vitien, ein Anstieg des LV-Füllungsdruckes oder eine fehlende myokardiale Reserve (• Abb. 2; $[57,58])$.

Die dynamische Stressechokardiographie liefert insbesondere bei unklarer Dyspnoe in Bezug auf den Schweregrad der systolischen LV-Dysfunktion oder der Mitralklappeninsuffizienz (MI) in Ruhe sowie bei unklarem rezidivierendem Lungenödem wichtige diagnostische Hinweise [5, 51]. Eine medikamentöse Belastung mit Dobutamin ist nicht anzuraten, da die medikamentöse Stimulation einer unphysiologischen Belastung entspricht [5, 58]. Der 


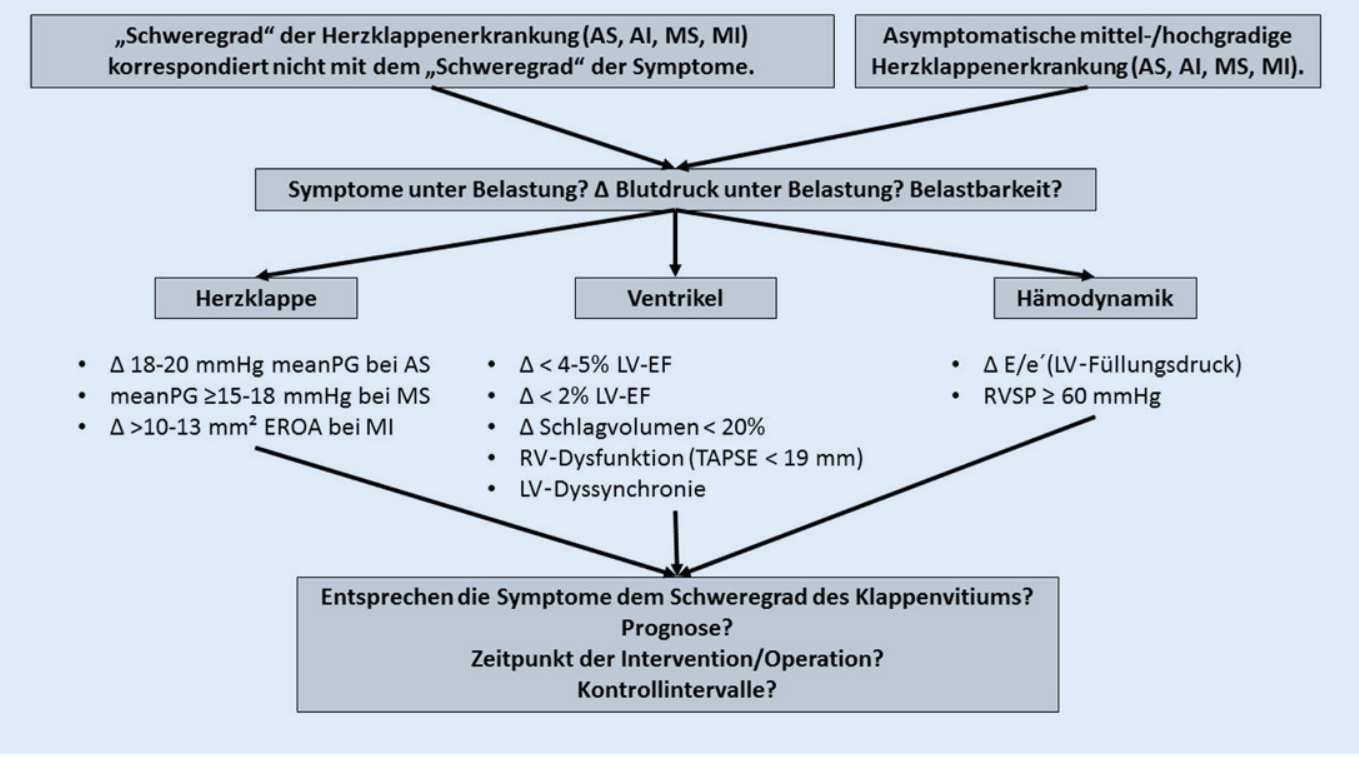

Abb. $2 \varangle$ Schematische Darstellung zur Charakterisierung pathologischer Befunde bei der Stressechokardiographie bei Herzklappenerkrankungen unter Berücksichtigung der Symptomatik, der Blutdruckreaktion sowie der Belastbarkeit im Hinblick auf die Therapie
Schweregrad einer MI kann sowohl bei veränderter Vorlast als auch bei veränderter Nachlast variieren $[5,58]$. Wenn die MI in Ruhe nicht hochgradig erscheint, sollten zusätzlich eine vollständige semiquantitative und quantitative Quantifizierung des Klappenvitiums [18, 43, 50, 51, 57, 65, 108] während der Belastung und unmittelbar nach der maximalen Belastung erfolgen. Einschränkend ist zu erwähnen, dass sich die exakte Evaluation der Ml ab einer Herzfrequenz $>$ 100/min als schwierig gestaltet. Bei der Stressechokardiographie bei Patienten mitMI sollten insbesondere der RVSP sowie die LV-Funktion aus prognostischen Gründen analysiert werden $[5,43,55,58]$. Bei Patienten mit primärer MI deuten unter Belastung die Zunahme des Schweregrads ( $\geq 1$ Stufe) $[43,58,65]$, ein Anstieg des RVSPs $\geq 60 \mathrm{~mm} \mathrm{Hg}$ [108], das Fehlen der Zunahme der LVEF um weniger als $5 \%$ oder des Global Longitudinal Strains (GLS) um weniger als $2 \%$ des Absolutwertes [18, 53] und der rechtsventrikulären kontraktilen Reserve (TAPSE < 18 mm) [50] auf eine ungünstige Prognose hin. Patienten mit sekundärer MI mittleren Schweregrades mit Indikation zur operativen Koronarrevaskularisation sollten ebenfalls präoperativ einer Stressechokardiographie unterzogen werden, um über einen kombinierten Eingriff von Bypass- und Klappenchirurgie zu entscheiden [5]. Eine Zunahme des MISchweregrads sowie ein Anstieg des RVSPs $\geq 60 \mathrm{~mm} \mathrm{Hg}$ gelten als Prädiktoren für eine ungünstige Prognose bei nicht durchgeführter Klappentherapie [5, 50, 51, 53, 79].

Bei Patienten mit Mitralklappenstenose (MS) sollte primär eine dynamische Stressechokardiographie sowohl bei asymptomatischen Patienten mit hochgradiger Stenosierung als auch bei symptomatischen Patienten mit gering- bzw. mittelgradiger MS durchgeführt werden [58]. Dies ist insbesondere bei einer Diskrepanz zwischen klinischer Symptomatik und echokardiographisch bestimmtem MSSchweregrad indiziert $[5,12,79]$. Eine hämodynamisch bedeutsame MS liegt vor, wenn ein mittlerer transmitraler Gradient $>15 \mathrm{~mm} \mathrm{Hg}$ und ein RVSP $>60 \mathrm{~mm} \mathrm{Hg}$ in Ruhe bzw. unter Belastung gemessen werden können $[9,96]$. Die Messung des RVSPs unter Dobutamin ist nur unzureichend evaluiert und somit nicht anzuraten [5, 79]. Bei Patienten mit asymptomatischer MS und einer Klappenöffnungsfläche zwischen 1,0 und $1,5 \mathrm{~cm}^{2}$ ist eine Evaluation durch eine Stressechokardiographie vor einer geplanten Schwangerschaft oder elektiven nichtkardialen Operationen mit langen Narkosezeiträumen indiziert [58].

Bei der Aortenklappeninsuffizienz (Al) wird eine Stressechokardiographie lediglich zur Demaskierung von Symptomen unter Belastung bei im Alltag beschwerdefreien Patienten mit echokardiographisch hochgradigem Klappenvitium empfohlen $[5,79]$. Aufgrund der schlechten Langzeitprognose einer hochgradigen Al wird bei Symptomen im Rahmen einer Belastungsuntersuchung eine operative Therapie empfohlen. Eine Stressechokardiographie zur Evaluation des Schweregrades einer $\mathrm{Al}$ ist hingegen nicht etabliert, da bei Herzfrequenzanstieg eine korrekte Beurteilung des Al-Schweregrades durch die verringerte Diastolendauer erschwert ist [58].

Bei Patienten mit symptomatischer hochgradiger Aortenklappenstenose (AS) ist eine Stressechokardiographie kontraindiziert [13, 14, 52, 54, 67], und es besteht eine Indikation zur Therapie der AS. Zirka ein Drittel der asymptomatischen Patienten mit hochgradiger AS entwickelt erstmalig Symptome unter moderater dynamischer Belastung im Rahmen eines Stresstestes [52, 54]. Bei der Stressechokardiographie sollte zur Analyse des ASSchweregrades in Ruhe und auf jeder Belastungsstufe der mittlere und maximale transvalvuläre Gradient mittels CW-Doppler neben der Analyse der LV-Funktion und des pulmonalarteriellen Drucks bestimmt werden [54]. Ein Anstieg des mittleren transvalvulären Gradienten über der Aortenklappe $\geq 18-20 \mathrm{~mm} \mathrm{Hg}[52,67]$, eine fehlende myokardiale Reserve (fehlender Anstieg der LVEF > 5\%) [54] und eine neu aufgetretene pulmonale Hypertonie (RVSP > 60 mm Hg) unter Belastung [58] sind Befunde, die mit einer erhöhten kardiovaskulären Mortalität assoziiert sind. Somit ist bei relevantem Anstieg des mitt- 
leren transvalvulären Gradienten auch bei asymptomatischen AS-Patienten ein therapeutisches Vorgehen zu diskutieren $[5,79]$.

Eine besondere Indikationsstellung zur Stressechokardiographie bei AS-Patienten ist die LF-LG-AS bei reduzierter LVEF, die durch eine effektive Klappenöffnungsfläche (KÖF) $<1,0 \mathrm{~cm}^{2}$, einen mittleren transvalvulären Gradienten $<40 \mathrm{~mm} \mathrm{Hg}$ und einen Schlagvolumen-Index (SVI) $<35 \mathrm{ml} / \mathrm{m}^{2}$ charakterisiert ist $[13,14]$. Diese Konstellation kann bei eingeschränkter LV-Funktion (LVEF $<50 \%$ ) als fixierte oder Pseudo-LF-LG-AS oder bei erhaltener Pumpfunktion (EF $\geq 50 \%$ ) als sog. paradoxe LF-LG-AS vorliegen. Eine PseudoAS ist eine Konstellation, bei der sich die effektive KÖF durch Verbesserung der LVKontraktion vergrößert, also bei der eine dynamische Veränderung des AS-Schweregrades vorliegt. Zur Differenzierung zwischen den AS-Typen sowie zur Unterscheidung von fixierter AS und PseudoAS wird eine niedrig dosierte DobutaminStressechokardiographie (Low-dose-Dobutamin-Stressechokardiographie-Startdosis: 5, 10, 15 und $20 \mu \mathrm{g} / \mathrm{kg} / \mathrm{min}$; jeweils für 5-8 min pro Belastungsstufe) bei AS-Patienten diskutiert [5]. Bei dieser Stresstestung müssen auf jeder Belastungsstufe ein PW-Dopplerspektrum im LV-Ausflusstrakt (LVOT) und ein transvalvuläres CW-Doppler-Spektrum durch die Aortenklappe sowie eine Dokumentation der LV-Funktion aufgezeichnet werden [58]. Durch eine Dobutamin-induzierte Kontraktilitätssteigerung des LV-Myokards bei nahezu gleicher Herzfrequenz soll ein Anstieg des Schlagvolumens um mehr als 20\% des Ruhewertes zu einem konsekutiven Anstieg des transvalvulären Druckgradienten führen. Da sich die transvalvuläre Flussrate (= Volumenflussbedingungen) mit zunehmender Herzfrequenz signifikant verändert, soll sich bei der Low-dose-Dobutamin-Stressechokardiographie die Herzfrequenz maximal um nur 10 Schläge/min verändern, um vergleichbare Flussbedingungen bei Zunahme der Kontraktilität zu gewährleisten. Bleibt die unter Belastung berechnete effektive KÖF bei LF-LG-AS-Patienten mit reduzierter LVEF gleich, handelt es sich um eine sog. fixierte AS, vergrößert sich die KÖF um mehr als $20 \%$ auf Werte $>1 \mathrm{~cm}^{2}$ handelt es sich um eine sog. Pseudo-AS [13, 14]. Aufgrund der ungünstigen Langzeitprognose bei Patienten sowohl mit fixierter wie auch paradoxer LF-LG-AS wird in beiden Fällen eine Therapie der AS diskutiert [5].

Die Stressechokardiographie ist zur Abklärung von Prothesendysfunktionen - insbesondere bei Vorliegen einer Diskrepanz zwischen echokardiographischer Schweregradbestimmung der Stenosierung (PPM $=$ "patient prosthesis mismatch“) oder Insuffizienz und der geschilderten klinischen Symptomatik des Patienten - indiziert [59, 87, 120]. Sowohl die dynamische als auch die Low-doseDobutamin-Stressechokardiographie können relevante Prothesenstenosen durch einen signifikanten Anstieg des PPM (> $20 \mathrm{~mm} \mathrm{Hg}$ bei Aortenklappenprothesen oder $>10 \mathrm{~mm} \mathrm{Hg}$ bei Mitralklappenprothesen) unter Belastung detektieren, die meist mit einem pathologischen Anstieg des RVSP (>60 $\mathrm{mm} \mathrm{Hg}$ ) einhergehen [59, 87, 120].

\section{Die Kontrastechokardiographie oder kontrastverstärkte Echokardiographie}

Die sog. „nicht-lungengängigen Ultraschallkontrastverstärker" sind zum Nachweis von intrakardialen und intrapulmonalen Shunts und zur Verstärkung von Dopplersignalen der Trikuspidalklappe indiziert. Nicht-lungengängige UEAs bestehen aus Gasbläschen, die aufgrund ihrer Größe die Lungenkapillaren nicht passieren können. Seitdem die Produktion von D-Galaktose (Echovist ${ }^{\mathrm{TM}}$ - Bayer Vital $\mathrm{GmbH}$, Leverkusen, Deutschland) eingestellt wurde, müssen nicht-lungengängige UEA selbst hergestellt werden. Hierzu werden $9 \mathrm{ml}$ einer sterilen $\mathrm{NaCl}-0,9 \%$-Lösung mit 0,5-1 ml Luft über einen 3-Wege-Hahn zwischen 2 Luer-Lock-Spritzen mehrmals so rasch wie möglich hin und her bewegt (sog. Agitieren), und die dabei entstehende trübe Lösung wird unmittelbar mit möglichst geringem Druck injiziert, wobei sichtbare Bläschen nicht injiziert werden. Ist die Kontrastierung des rechten Vorhofes und rechten Ventrikels unzureichend oder zu kurz anhaltend, können durch Hinzufügen von $1 \mathrm{ml}$ Patientenblut zu $8 \mathrm{ml} \mathrm{NaCl-Lösung} \mathrm{und} 0,5 \mathrm{ml}$ Luft die
Kontrastverwirkung verbessert und die Dauer der Kontrastierung um etwa $20 \mathrm{~s}$ verlängert werden [45]. Zur Verstärkung von Dopplersignalen sollte die agitierte $\mathrm{NaCl}$-Lösung mit Blutzusatz bevorzugt werden.

Die sog. „lungengängigen Ultraschallkontrastverstärker (UEA - ,ultrasound enhancing agents')" bestehen aus einer stabilen Hülle aus Phospholipiden oder Albumin und einem in Plasma schwer löslichen Gas wie Schwefelhexafluorid oder Perflutren. Die Kontrastbläschen weisen eine mittlere Größe von 2-5 $\mu \mathrm{m}$ auf und können so die Lungenkapillaren passieren. Lungengängige UAEs werden deshalb auch als Linksherzkontrastmittel bezeichnet. Zugelassen sind alle 3 in Europa verfügbaren lungengängigen UEAs (Luminity $^{\mathrm{TM}}$ - Lantheus EU Limited, Shannon, Irland; Optison ${ }^{\mathrm{TM}}-\mathrm{GE}$ Healthcare AS, Oslo, Norwegen; SonoVue ${ }^{\mathrm{TM}}$ - Bracco International B.V., Amsterdam, Niederlande) zur Verbesserung der LV-Endokard-Erkennung, der sog. „left ventricular opacification" (LVO). Die EACVI (European Association for Cardiovascular Imaging) hat als Teil der Europäischen Gesellschaft für Kardiologie (ESC) 2017 über die Zulassung hinausgehende Empfehlungen zum klinischen Einsatz der lungengängigen UEAs veröffentlicht [99]. Die - Tab. 2 listet den Empfehlungsgrad und die Evidenz für die 10 wichtigsten Indikationen (Auswahl) auf.

Aktuell ergibt sich auch in der Pandemiesituation bei COVID-19-bedingten Pneumonien infolge von SARS-CoV-2 eine Indikation für die Kontrastechokardiographie. In vielen Fällen wird eine Troponinerhöhung beobachtet, die eine genaue LVWandbewegungsanalyse durch UEAs bei eingeschränktem Schallfenster notwendig macht. Eine Takotsubo-Kardiomyopathie kann mit der Kombination von kontrastverstärkter Wandbewegungsanalyse und Perfusionsdarstellung nachgewiesen werden. Weitere Indikationen stellen der Verdacht auf eine Plaqueruptur, spontane Koronardissektion sowie eine Myokarditis mit und ohne mikrovaskuläre Obstruktion dar. Während die FDA (Food and Drug Administration) für die Gruppe der lungengängigen UEAs als einzige Kontraindikation eine bekannte Allergie gegen die Substanz oder ihre Komponenten nennt, führt die Europäische Arzneimittel- 
Tab. 2 Empfehlungsgrad und Evidenz der wichtigsten Indikationen zum klinischen Einsatz von lungengängigen Ultraschallkontrastverstärkern (UEA)

\begin{tabular}{|c|c|}
\hline $\begin{array}{l}\text { Empfehlungsgrad/ } \\
\text { Evidenzgrad }\end{array}$ & Empfehlung \\
\hline \multirow[t]{3}{*}{ Klasse I, Level A } & $\begin{array}{l}\text { Im Rahmen der Beurteilung der regionalen Myokardfunktion sollten UEAs erwogen werden, wenn } 2 \text { oder mehr benachbarte } \\
\text { Wandsegmente nicht ausreichend sichtbar sind }{ }^{\mathrm{a}} \text { und die weitere Behandlung davon abhängt, ob regionale Wandbewegungs- } \\
\text { störungen vorliegen oder nicht }\end{array}$ \\
\hline & $\begin{array}{l}\text { Im Rahmen der Stressechokardiographie sollten UEAs erwogen werden, wenn bei der regionalen Wandbewegungsanalyse } 2 \\
\text { oder mehr benachbarte Wandsegmente nicht ausreichend beurteilt werden können }\end{array}$ \\
\hline & $\begin{array}{l}\text { Die myokardiale Kontrastechokardiographie (MCE) mit UEAs sollte erwogen werden bei Patienten mit Dobutamin-, Vasodila- } \\
\text { tator-Stressechokardiographie und Hochrisikopatienten mit nichtpharmakologischem Stress zur Verbesserung von Diagnose } \\
\text { und Risikostratifizierung über die Wandbewegungsanalyse hinaus }{ }^{b}\end{array}$ \\
\hline \multirow[t]{2}{*}{ Klasse I, Level B } & $\begin{array}{l}\text { Bei Verdacht auf Vorliegen eines linksventrikulären Thrombus sollten UAEs erwogen werden, wenn der Thrombus weder ein- } \\
\text { deutig dokumentiert noch sicher ausgeschlossen werden kann }{ }^{\text {a }}\end{array}$ \\
\hline & $\begin{array}{l}\text { Bei Verdacht auf Vorliegen einer apikalen hypertrophen Kardiomyopathie, eines Divertikels, einer „Non-compaction“-Kardio- } \\
\text { myopathie, eines Pseudoaneurysmas oder einer Myokardruptur sollten UAEs erwogen werden, wenn die Pathologie weder } \\
\text { eindeutig dokumentiert noch sicher ausgeschlossen werden kanna }\end{array}$ \\
\hline \multirow[t]{2}{*}{ Klasse Ila, Level B } & $\begin{array}{l}\text { Unabhängig von der Bildqualität sollten UAEs bei der quantitativen Analyse des linksventrikulären Volumens und der Funktion } \\
\text { erwogen werden, wenn die klinische Behandlung von einer genauen Bestimmung der Ejektionsfraktion abhängt wie unter } \\
\text { Therapie mit kardiotoxischen Medikamenten oder wenn die Implantation eines ICD- oder CRT-Gerätes erwogen wird }\end{array}$ \\
\hline & $\begin{array}{l}\text { Die myokardiale Kontrastechokardiographie kann erwogen werden zur Verbesserung der Erkennung der Vitalität von Myo- } \\
\text { kardsegmenten, insbesondere im Rahmen einer Dobutamin-Stressechokardiographie, wenn Wandsegmente mit erhaltener } \\
\text { Wanddicke unter Stressbedingungen keine Wanddickenzunahme aufweisen }\end{array}$ \\
\hline \multirow[t]{3}{*}{ Klasse Ila, Level C } & $\begin{array}{l}\text { Zum Nachweis von Thromben im linken Herzohr im Rahmen einer transösophagealen Echokardiographie können UAEs erwo- } \\
\text { gen werden, wenn die Untersuchung nicht aussagekräftig ist }{ }^{\mathrm{a}}\end{array}$ \\
\hline & $\begin{array}{l}\text { Zur Analyse der Gewebeperfusion von kardialen Raumforderungen mittels myokardialer Kontrastechokardiographie können } \\
\text { UAEs erwogen werden, wenn eine kardiale MRT nicht verfügbar oder nicht aussagekräftig ist }\end{array}$ \\
\hline & $\begin{array}{l}\text { Zur Beurteilung der Pathologie der Aorta bei akutem Aortensyndrom und vor interventionellen Eingriffen an der Aorta können } \\
\text { UAEs erwogen werden, wenn die Dopplersignale oder 2-D-Bildgebung nicht aussagekräftig sind }\end{array}$ \\
\hline \multirow[t]{3}{*}{ Klasse III, Level B } & $\begin{array}{l}\text { Die Verwendung von UEAs bei der Analyse der regionalen linksventrikulären Funktion bei gutem Schallfenster wird außerhalb } \\
\text { der Stressechokardiographie nicht empfohlen }\end{array}$ \\
\hline & $\begin{array}{l}\text { Die quantitative Analyse von linksventrikulärem Volumen und Funktion mittels 3-D-Echokardiographie mit UAEs wird derzeit } \\
\text { aufgrund der limitierten Datenlage noch nicht empfohlen }\end{array}$ \\
\hline & $\begin{array}{l}\text { Im Rahmen der Stressechokardiographie wird die 3-D-Echokardiographie mit UAEs aufgrund der limitierten Datenlage nicht } \\
\text { empfohlen }\end{array}$ \\
\hline
\end{tabular}

Agentur (EMA) für Optison noch eine pulmonale Hypertonie (sPAP > $90 \mathrm{~mm} \mathrm{Hg}$ ) und für SonoVue zusätzlich zur pulmonalen Hypertonie einen bekannten RechtsLinks-Shunt, einen unkontrollierten systemischen Hochdruck und ein ARDS auf. Shunts und ARDS werden bei den beiden anderen Substanzen mit einem Warnhinweis aufgeführt. Das Risiko für schwere anaphylaktische Reaktionen nach UEAs wird mit 1:10.000 Untersuchungen angegeben und liegt damit in der gleichen Größenordnung wie bei MRT-Kontrastmitteln und 10-fach niedriger als bei Röntgenkontrastmitteln [77, 90, 99, 111]. Die Applikation setzt somit immer eine Risiko-Nutzen-Abwägung voraus sowie eine schriftliche Aufklärung in Analogie zur Verwendung von jodierten Kontrastmitteln.
Da das Gas lungengängiger UEAs pulmonal eliminiert wird, können UEAs im Gegensatz zu den jodierten und in der MRT-Diagnostik verwendeten Kontrastmitteln auch bei hochgradigen Nierenfunktionsstörungen eingesetzt werden. Hauptbedenken bei dem Einsatz von lungengängigen UEAs stellt das Auftreten einer pseudoanaphylaktischen Reaktion dar, der sog. CARPA („'complement activation-related pseudoallergy"), die auch für jodierte Kontrastmittel beschrieben ist. Durch die liposomale Struktur der Kontrastbläschen kann eine ComplementAktivierung über den klassischen und alternativen Weg erfolgen und bereits bei der ersten Injektion auftreten. Die Symptome können mit Niesen, Juckreiz, Kribbeln der Haut, Urtikaria sowie Rückenoder Flankenschmerzen milde bis mäßig- gradig ausgeprägt sein. Schwergradige Reaktionen gehen einher mit Hypotonie bis Schock, Luftnot, Hypoxie bis Atemstillstand, Brustschmerz, Hauterythem, Arrhythmien und Krampfanfällen. Die Reaktion ist abhängig von der Geschwindigkeit der Applikation (Bolus vs. Infusion), der Gesamtdosis sowie möglicherweise auch mit Complement-erhöhenden Erkrankungen wie einer Sichelzellanämie oder der seltenen paroxysmalen nächtlichen Hämoglobinurie. Eine Anamnese auf Nahrungsmittel- oder Arzneimittelallergien sowie eine schriftliche Aufklärung des Patienten sollten vor Applikation erfolgen. Zur Therapie akuter anaphylaktischer Reaktionen sollten alle Voraussetzungen im Notfall im Ultraschalllabor vorhanden sein. Notfallmedikamente (Suprarenin, $\mathrm{H}_{1}-, \mathrm{H}_{2}$-Antagonisten, Steroide, inhalative 
$\beta$-Mimetika, Infusionslösungen) müssen daher bereit liegen [90, 100, 115]. Der Untersucher sollte im "advanced life support" (ALS), die Assistenz im "basic life support" (BLS) geschult sein. Die Anforderungen an das Personal entsprechen somit denen von Herzkatheterlaboren.

Zur Visualisierung des Kontrasteffektes lungengängiger UAEs müssen technische Voraussetzungen gegeben sein. Die mit Gas gefüllten Bläschen schwingen bei erniedrigter Schallenergie im Ultraschallfeld, wobei bei Verwendung von harmonischer Bildgebung die durch die Bläschengröße definierte Resonanzfrequenz der Empfangsfrequenz der Standardechokardiographie entspricht (z.B. 1,7/3,4 MHz). Zur Reduktion von Artefakten bieten viele Hersteller von Ultraschallgeräten "LVO-Programme" an („tissue harmonic B-mode" mit Hochpassfilter). Wird die Sendeleistung mittels des mechanischen Index eingestellt, so ist ein intermediärer mechanischer Index von 0,2-0,3 (0,5) sinnvoll. Ursachen für schlechte Bilder in der Kontrastechokardiographie sind die Zerstörung von Kontrastbläschen im Nahfeld, die höhere Signalabschwächung im Fernfeld sowie das nicht optimale Signal-Rausch-Verhältnis zwischen kontrastmittelgefüllter Kammer und den noch vorhandenen fundamentalen Signalen des Myokards. Zur Reduktion von Nahfeldartefakten und zur Erhöhung der Sensitivität für Kontrastsignale wurden die sog. kontrastspezifischen Programme wie die Multipuls-Technik entwickelt (Phaseninversion, Amplitudenmodulation, ,"Cadence Imaging" u.a.). Bei der Phaseninversion wird durch Subtraktion der linearen Signale zweier phasenverschobener Bilder das fundamentale Signal des Myokards weitestgehend eliminiert. Die nichtlinearen Signale der Kontrastbläschen werden nach Subtraktion für den Bildaufbau verwendet. Hierdurch wird die Analyse der Myokardperfusion möglich. Der mechanische Index kann für Perfusionsanalysen auf Werte zwischen 0,1 und 0,2 abgesenkt werden. Die sog. „Low Mechanical Index“Technik wird derzeit für alle Indikationen der lungengängigen Kontrastmittel empfohlen. Die notwendigen technischen Voraussetzungen für die Kontrastechokardiographie sind in der Regel nur auf sog. "High-End“-Geräten implementiert.
Aktuell sind Programme mit 3 Impulsen verfügbar, die Phaseninversion und Amplitudenmodulation kombinieren und verstärkte ultraharmonische Frequenzen bei der 1,5-fachen Sendefrequenz zum Bildaufbau verwenden. Vorteilhaft sind die völlige Elimination von harmonischen Signalen des Myokards und die hohe Eindringtiefe mit dieser Technik, nachteilhaft die geringere örtliche Auflösung im Vergleich zur alleinigen Phaseninversion bei gleicher Bildrate von 24 bis 30 Bildern/s [99].

Die Bolusinjektion eines lungengängigen UEAs ist geeignet zur Verbesserung der LV-Endokard-Abgrenzung für die Wandbewegungsanalyse in Ruhe und während Stressuntersuchungen, die Bestimmung von LV-Volumina und LVEF sowie zur Diagnose von ventrikulären Thromben oder strukturellen Herzerkrankungen wie der apikalen hypertrophen Kardiomyopathie. Die Infusion des UAEs mithilfe einer Infusionspumpe, die durch eine kontinuierliche Schwenkbewegung der Spritze eine Trennung von gasgefüllten Bläschen und $\mathrm{NaCl}$-Lösung verhindert (VueJect-Pumpe), ist empfohlen für die myokardiale Kontrastechokardiographie im Rahmen der Stressechokardiographie sowie zur Analyse der Perfusion myokardialer Raumforderungen [99, 100, 111, 116]. Der erste Schritt einer kontrastverstärkten Untersuchung besteht in der Anwahl eines Kontrastprogramms. Erfolgt eine Bolusinjektion, so kann durch mehrmalige tiefe Inspiration oder durch langsame Nachinjektion von $5 \mathrm{ml} \mathrm{NaCl}$ 0,9\%-Lösung die Anflutung des UAEs beschleunigt werden. Im nächsten Schritt erfolgt die Optimierung des Kontrastbildes. Die Registrierung der Bilder sollte bei einer Konzentration des Kontrastmittels erfolgen, die das beste Signal-RauschVerhältnis ergibt. Eine zu hohe Konzentration ist an einer zu hohen Signalintensität im Nahfeld („blooming ${ }^{\prime \prime}$ ) und einem Abschattungsartefakt im Fernfeld des Sektors („,attenuation, shadowing ${ }^{\prime \prime}$ ) erkennbar und typisch für die ersten Sekunden nach Bolusinjektion. Eine Unterdosierung ist an einer nachlassenden Signalintensität des UEAs mit Ausbildungen von Verwirbelungen erkennbar. Sie setzt abhängig vom gewählten Kontrastprogramm zwischen 30 s und 2 min nach Bolusinjektion ein. Die
Anpassung der Sendeleistung des Schallkopfes erfolgt mithilfe des mechanischen Index. Bei optimaler Sendeleistung ist in den apikalen Schnittebenen die Zerstörung von Kontrastbläschen im Bereich der Herzspitze minimiert und gleichzeitig eine ausreichende Signalintensität auch noch aus den Klappenring-nahen Abschnitten des linken Vorhofs vorhanden. Der Fokus wird bei Verwendung der Phaseninversion im Bereich der Mitralklappe positioniert, bei Verwendung von Ultraharmonics in Höhe der mittleren Wandsegmente und sollte bei Beurteilung apikaler Strukturen in Richtung Herzspitze verschoben werden. Die Optimierung der Scanebene richtet sich u.a. nach möglichst geringer Verkürzung der Bildebenen, nach der Vermeidung von Rippenartefakten und nach der Minimierung von Papillarmuskelanschnitten. Zur Optimierung des Signal-Rausch-Verhältnisses kann bei Verwendung der "Low mechanical index"Multipuls-Technik durch Abgabe kurzer Impulse mit hoher Sendeleistung (= sog. "flash") das Perfusionssignal im Myokard reduziert werden. Es ist empfehlenswert, bei Vorliegen von Sinusrhythmus mehrere Kontrastmittelsequenzen der 3 apikalen Schnittebenen mit wenigstens 2 RR-Zyklen vor und 5 RR-Zyklen nach dem Flash zu registrieren.

Die Ausbildung in der kontrastverstärkten Echokardiographie sollte unter Anleitung eines in der Kontrastechokardiographie erfahrenen Untersuchers erfolgen. Die aktualisierte 2. Auflage des Curriculum Kardiologie von 2020 [34] führt unter den theoretischen Kenntnissen, die im Rahmen der echokardiographischen Ausbildung zum Kardiologen erworben werden sollen, die Rechts- und Linksherzkontrast-Echokardiographie auf. Für die praktischen Fähigkeiten soll der Weiterzubildende lernen, eigenständig die Indikation für die Kontrastechokardiographie zu erkennen, die Technik durchzuführen, die Befunde zu interpretieren, Komplikationen zu beherrschen und die Kompetenz durch regelmäßige Durchführung zu bewahren (Level III) [90, 116]. Gegenüber der 1. Auflage von 2014 wurde eine Mindestzahl an Untersuchungen nicht mehr aufgenommen. Die amerikanischen Fachgesellschaften ACC/AHA/ASE $[90,116]$ von 2019 empfehlen dagegen weiterhin ei- 
ne Mindestzahl von 100 eigenständigen Untersuchungen. Die Durchführung der myokardialen Kontrastechokardiographie im Rahmen der Stressechokardiographie erfordert ein zusätzliches Training [90, 115, 116].

\section{Die transthorakale und transösophageale 3D- Echokardiographie}

Die multidimensionale (3D-)Echokardiographie setzt das Arbeiten mit einem sog. 3D-Volumen-Transducer voraus, durch den ein vollständiger Volumendatensatz kardialer Strukturen aufgenommen werden kann. Innerhalb dieses Volumendatensatzes können dann beliebige Schnittebenen im Realtime-Modus sowie in der Nachbearbeitung zu quantitativen Analysen eingestellt werden. Ebenso können mit einem Volumen-Transducer simultane Schnittebenen im Live-Modus akquiriert werden, wobei der perpendikuläre biplane Modus sowie der triplane Modus mit in $60^{\circ}$ Abständen eingestellten Schnittebenen am häufigsten verwendet wird (• Abb. 3). Diese relativ neuen Modalitäten - die multiplane und multidimensionale Technik tragen zu erheblichen Verbesserungen der echokardiographischen Diagnostik in TTE und TEE bei [60].

Die grundsätzliche technische Herausforderung der Bildakquisition mit Volumen-Transducern besteht in der Aufteilung der Sende- und Empfangsleistung auf mehrere Schnittebenen oder ein komplettes Volumen, was natürlich einen Einfluss auf Bildqualität bzw. die räumliche und zeitliche Auflösung beinhaltet. Die multiplane Technik ist aus diesem Grund bei Patienten mit eingeschränkten Schallbedingungen gegenüber der multidimensionalen Technik zu bevorzugen, da bi- oder triplane Schnittbilder aufgrund schärferer und eindeutiger Konturerkennung in der Regel besser sind und höherer zeitliche Auflösungen aufweisen als entsprechende Schnittbilder aus 3D-Datensätzen, sodass dadurch auch verlässliche Messungen vorgenommen werden können, sofern die richtigen Schnittebenen dargestellt wurden $[60,69]$. Die multiplane Echokardiographie eignet sich exzellent zur Standardisierung der Schnittebenen (z. B. die genaue perpendikuläre Anlotung von parasternal langer Achse und kurzer Achsen sowie die exakte Einstellung der LV-Cavumspitze und der Mitralklappe in der Mitte des Sektors in allen 3 apikalen Schnittebenen in der TTE), zur eindeutigen Klärung der kardialen Anatomie (z. B. Darstellung des anatomischen Verlaufes von Koronararterien in der TTE und Darstellung von oberen und unteren Lungenvenen in der TEE) sowie der perpendikulären Einstellung kardialer Strukturen (z. B. Klappenöffnungsflächen bei AS und MS bzw. Regurgitationsöffnungsflächen bei Al und MI in der TTE und TEE) (- Abb. 3 und 4). Aufgrund der exakten Standardisierung der apikalen Schnittebenen sind zudem exakte triplane Volumenanalysen des linken Ventrikels sowie des linken und rechten Atriums möglich. Die simultanen Schnittbilddarstellungen erleichtern auch die kardialen Volumenbestimmungen bei Herzrhythmusstörungen - insbesondere bei Vorhofflimmern und häufiger ventrikulärer Extrasystolie.

Die multidimensionale (3D-)Echokardiographie ermöglicht neue Dimensionen der Echokardiographie z.B. mit sog. Enface-Blicken auf kardiale Strukturen [73, 75, 76]. Erstens können Schnittebenen eingestellt werden, die in der konventionellen TTE und TEE nicht akquiriert werden können (als Beispiel sei der Kurzachsenschnitt der Trikuspidalklappe genannt). Zweitens sind aus nicht standardisierten Anlotungen Bildakquisitionen möglich, die Auswertungen in standardisierten Ebenen in der Nachbearbeitung ermöglichen (als Beispiel sei die Analyse der LVVolumina aus subkostalen 3D-Datensätzen genannt). Drittens können komplette Volumenanalysen kardialer Herzhöhlen vorgenommen werden, die in der konventionellen Schnittbildtechnik nicht dargestellt werden können (als Beispiel sei die Volumenanalyse des rechten Ventrikels genannt). Viertens können Oberflächenansichten, sog. „En-face-Views“ eingestellt werden, die der konventionellen 2D-Echokardiographie generell nicht zugänglich sind (als Beispiele seien hier der En-faceView auf die Trikuspidalklappe oder auf interatriale Septumdefekte genannt).

Aufgrund dieser Möglichkeiten spielt die echokardiographische 3D-Technik bei Volumenanalysen in der TTE sowie bei morphologischen Analysen des Herzens - primär bei Pathologien der LV- und
Klappenmorphologie - sowohl in der TTE, aber besonders in der TEE eine klinisch wichtige Rolle. Die TEE hat aufgrund ihres Schallmodus mit höheren Frequenzen grundsätzlich bessere Voraussetzungen für eine bessere räumliche Auflösung und damit bessere Bildqualität. Mit Einführung der 3D-Echokardiographie in die TEE ist sie dadurch im heutigen klinischen Alltag fest etabliert. Nahezu keine TEE-Untersuchung in modernen kardiologischen Zentren wird heute ohne 3D-Technologie durchgeführt. Insbesondere beim Monitoring von Interventionen zur Behandlung struktureller Herzerkrankungen ist die 3D-TEE als notwendige Voraussetzung akzeptiert.

Aufgrund des nahezu ubiquitären Vorhandenseins der 3D-Technikin der TEE sind bei TEE-Untersuchungen multiplane und multidimensionale Bildsequenzen in aktuellen standardisierten TEE-Dokumentationen integriert (-Abb.4). Es ist daher zu erwarten, dass sich auch bei Patienten mit guten Schallbedingungen in der TTE die 3D-Technik im klinischen Alltag etabliert wird (- Abb. 3). Im Folgenden werden die zusätzlich zum standardisierten Untersuchungsgang der konventionellen Echokardiographie notwendigen multiplanen und multidimensionalen Bildsequenzen für die TTE und TEE aufgeführt.

Aus praktischer Sicht muss bei der Bildakquisition multidimensionaler Bildsequenzen berücksichtigt werden, dass immer ein Kompromiss zwischen akquirierter Volumengröße und zeitlicher bzw. räumlicher Auflösung vorliegt. Dies bedeutet, dass generell im sog. RealTime-Verfahren die zeitliche Auflösung gesteigert werden kann, wenn die Volumensektoren verkleinert werden oder kleinere Zoomausschnitte für eine 3DSequenz gewählt werden. Weiterhin wird die räumliche Auflösung verbessert, wenn die Bildrate bei gleicher Volumengröße verringert wird. Um eine Verbesserung der räumlichen und zeitlichen Auflösung in einem Volumendatensatz zu erhalten, kann das sog. „Near-Real-Time“-Verfahren angewandt werden. Bei dieser Modalität werden 3D-Volumina aus mehreren Herzzyklen zusammengesetzt, wobei die einzelnen Teilvolumina eine möglichst hohe räumliche und zeitliche Auflösung vorweisen. Voraussetzung für dieses „Vo- 

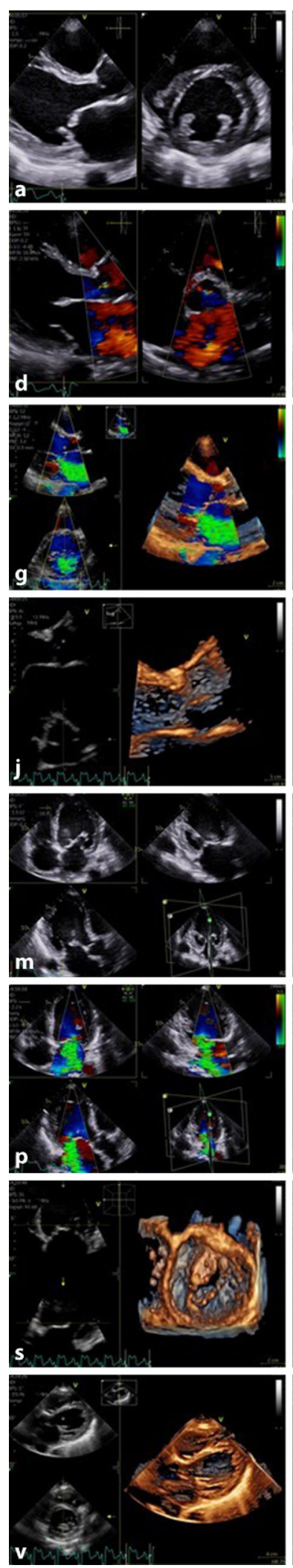
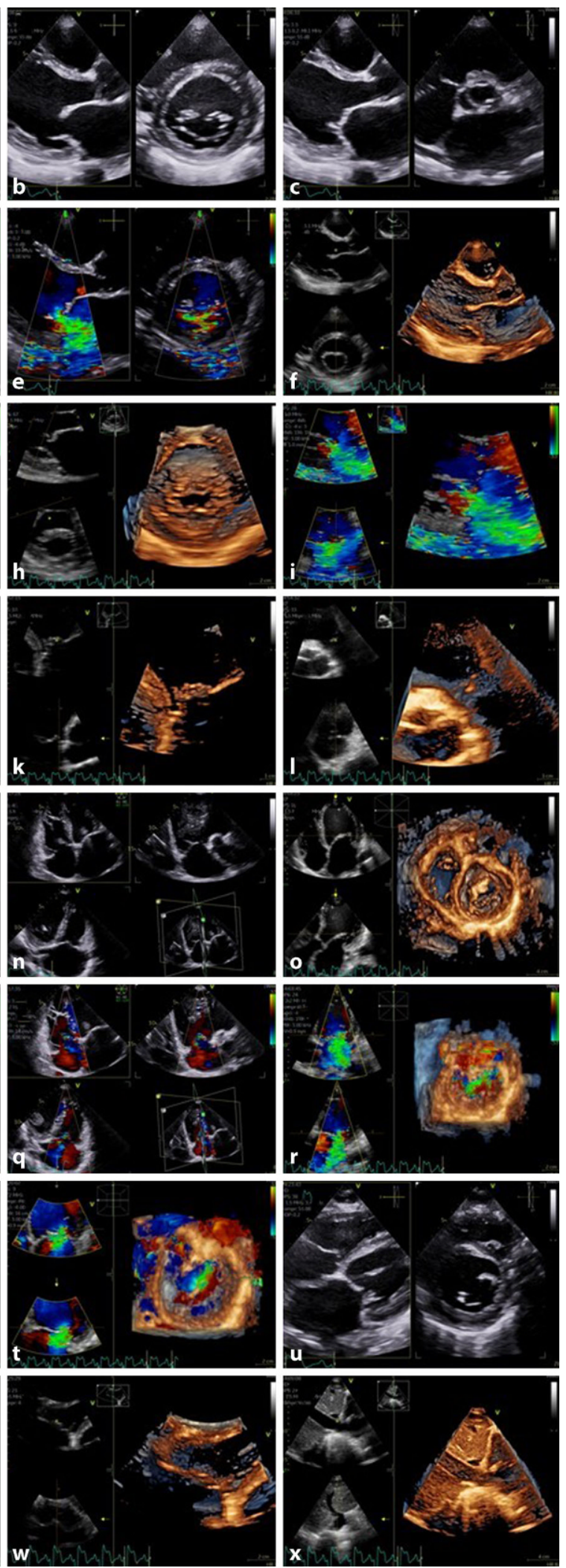

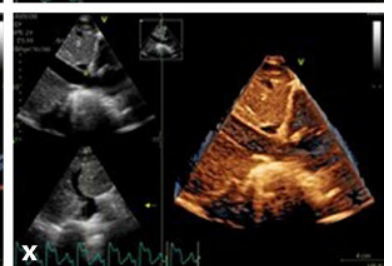

Abb. 34 Erweiterung der echokardiographischen Dokumentation des konventionellen standardisierten TTE-Untersuchungsgangs durch Bildsequenzen der multiplanen und multidimensionalen Echokardiographie. Genaue perpendikuläre Schnittebenen von Kurzachsenschnitten zur parasternal langen Achse in Höhe der Papillarmuskel (a), der Mitralklappe (b) und der Aortenklappe (c), apikale LV-Schnitte bzw. kraniale Schnitte durch die Pulmonalklappe sind zusätzlich möglich; farbkodierte biplane perpendikuläre Schnittebenen durch die Aortenklappe (d) und Mitralklappe (e), Dokumentationen weiterer Turbulenzen in parasternalen farbkodierten Sequenzen sind möglich; konventioneller 3D-Datensatz von parasternal (f); farbkodierter 3D-Datensatz von parasternal (g), bei Turbulenzen ist für jede Pathologie ein einzelner 3D-Datensatz zu akquirieren; gezoomter 3D-Datensatz der Mitralklappe im konventionellen (h) und farbkodierten 3D-Datensatz (i) von parasternal; gezoomte parasternale 3D-Datensätze der Aortenklappe (j), der Trikuspidalklappe (k) und Pulmonalklappe (l), bei Turbulenzen sind zusätzliche farbkodierte 3D-Datensätze möglich; simultane triplane konventionelle Darstellung des linken ( $\mathbf{m}$ ) und rechten ( $\mathbf{n}$ ) Ventrikels sowie der zugehörige 3D-Datensatz von apikal (o); simultane triplane farbkodierte Darstellung der Mitral- (p) und Trikuspidalklappeninsuffizienz (q) sowie der zugehörige farbkodierte 3D-Datensatz der Mitralklappeninsuffizienz von apikal (r), bei Turbulenzen sind zusätzliche farbkodierte 3D-Datensätze möglich; gezoomte apikale 3D-Datensätze konventionell (s) und farbkodiert (t) von der Mitralklappe, entsprechende Sequenzen können bei Klappenpathologien von allen weiteren Klappen dokumentiert werden; biplane Darstellung des linken Ventrikels von subkostal (u), weitere biplane Kurzachsendarstellungen sind möglich - auch im farbkodierten Modus bei Turbulenzen; konventioneller 3D-Datensatz von subkostal (v), zusätzliche farbkodierte 3D-Datensätze bei Turbulenzen sind möglich; gezoomter 3D-Datensatz der Trikuspidalklappe im konventionellen 3D-Datensatz (w) von subkostal, zusätzliche konventionelle und farbkodierte 3D-Datensätze bei Turbulenzen sind möglich; konventioneller 3D-Datensatz der Vorhöfe sowie V. cava inferior von subkostal (x) 

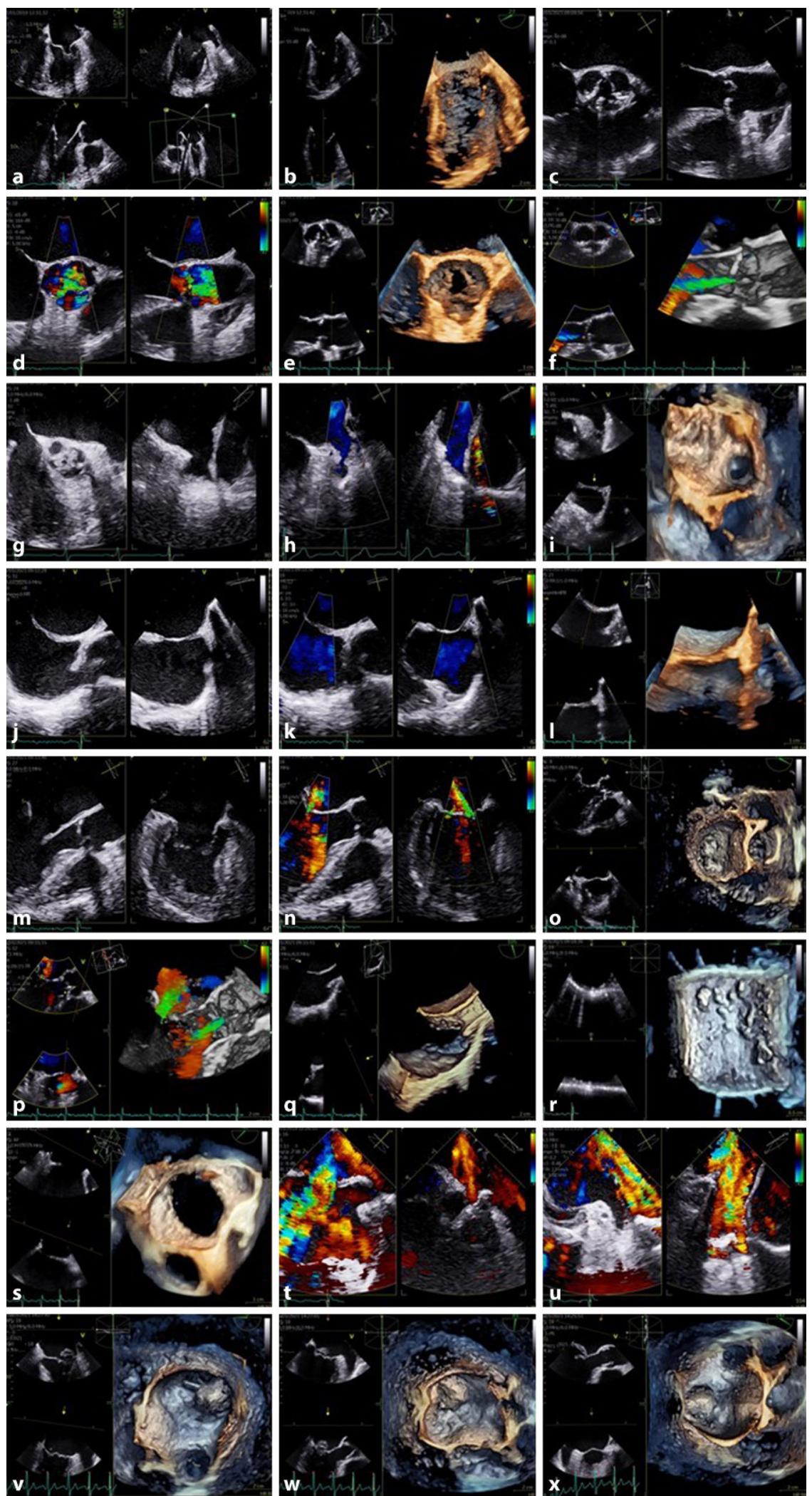
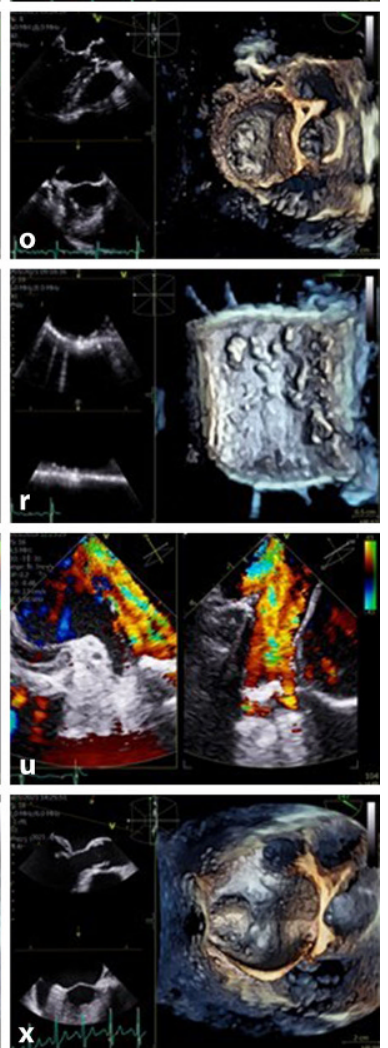

Abb. $4 \varangle$ Erweiterung der echokardiographischen Dokumentation des konventionellen standardisierten TEE-Untersuchungsgangs durch Bildsequenzen der multiplanen und multidimensionalen Echokardiographie. Triplane Darstellung des linken Ventrikels (a); 3D-Datensatz des linken Ventrikels (b), sowohl multiplan als auch multidimensional sind zusätzlich farbkodierte Sequenzen möglich; biplane konventionelle (c) und farbkodierte Darstellung der Aortenklappe (d); gezoomte konventionelle (e) und farbkodierte 3D-Darstellung der Aortenklappe (f); biplane konventionelle (g) und farbkodierte Darstellung des linken Vorhofohres (LAA) (h); gezoomte konventionelle 3D-Darstellung des LAA (i), biplane und 3D-Sequenzen unter LAA-Opazifizierung mit Kontrast sind zusätzlich möglich; biplane konventionelle (j) und farbkodierte Darstellung des interatrialen Septums (IAS) (k); gezoomte konventionelle 3D-Darstellung des IAS (I), biplane und 3D-Sequenzen des IAS mit nicht lungengängigem Kontrastmittel sind zusätzlich möglich; biplane konventionelle $(\mathrm{m})$ und farbkodierte Darstellung der Mitralklappe (n); gezoomte konventionelle (o) sowie farbkodierte 3D-Darstellung der Mitral- und Aortenklappe (p); bicavaler konventioneller 3D-Datensatz beider Hohlvenen (q);3-D-Datensatz der Aorta descendens (r), ggf. sind mehrere 3D-Datensätze der Aorta descendens zu dokumentieren; im Falle von Kommunikationsdefekten - 3D-Datensatz des IAS bei Septum-secundum-Defekt (s) sind mindestens die Einmündungen beider rechten $(\mathbf{t})$ und linken Lungenvenen ( $\mathbf{u})$ biplan zu dokumentieren, zusätzliche konventionelle und farbkodierte 3D-Datensätze der Lungenveneneinmündungen können dokumentiert werden; im Falle von Mitralklappenpathologien mit Therapienotwendigkeit sind wegen Minimierung von Artefakten (z. B. Schlagartefakte) mehrere 3D-Dokumentationen der Mitralklappe zu dokumentieren - primäre Schallebene jeweils 4-Kammer-Blick (v), 2-Kammer-Blick (w) und lange Achse (x), in allen Einstellungen können zusätzlich biplane konventionelle und farbkodierte Sequenzen sowie farbkodierte 3D-Datensätze aufgenommen werden 
Tab. 3 Herstellerspezifische repräsentative longitudinale Strain-Werte bei Gesunden (Mean) mit Standardabweichung (SD) und Untergrenze des Normalbereichs (LLN). (Nach [112])

\begin{tabular}{|c|c|c|c|c|c|}
\hline Vendor & Software & $n$ & Mean & SD & LLN \\
\hline Varying & Meta-Analysis & 2597 & $-19,7 \%$ & - & NA \\
\hline \multirow{4}{*}{$\begin{array}{l}\text { GE Health- } \\
\text { care (Germa- } \\
\text { ny), Solingen } \\
\text { Deutschland }\end{array}$} & EchoPAC BT 12 & 247 & $-21,5 \%$ & $2,0 \%$ & $-18 \%$ \\
\hline & EchoPAC BT 12 & 207 & $-21,2 \%$ & $1,8 \%$ & $-18 \%$ \\
\hline & EchoPAC BT 12 & 131 & $-21-2 \%$ & $2,4 \%$ & $-17 \%$ \\
\hline & EchoPAC 110,1.3 & 333 & $-21,3 \%$ & $2,1 \%$ & $-17 \%$ \\
\hline $\begin{array}{l}\text { Philips } \\
\text { Health } \\
\text { Systems } \\
\text { (Germany), } \\
\text { Hamburg, } \\
\text { Deutschland }\end{array}$ & QLAB 7,1 & 330 & $-18,9 \%$ & $2,5 \%$ & $-14 \%$ \\
\hline $\begin{array}{l}\text { Toshiba Me- } \\
\text { dical Systems } \\
\text { (aktuell Ca- } \\
\text { non Medical } \\
\text { Systems } \\
\text { - Germa- } \\
\text { ny) Neuss, } \\
\text { Deutschland }\end{array}$ & UltraExtend & 337 & $-19,9 \%$ & $2,4 \%$ & $-15 \%$ \\
\hline \multirow{2}{*}{$\begin{array}{l}\text { Siemens } \\
\text { Healthcare, } \\
\text { Erlangen, } \\
\text { Deutschland }\end{array}$} & VVI 1,0 & 116 & $-19,8 \%$ & $4,6 \%$ & $-11 \%$ \\
\hline & VVI 1,0 & 82 & $-17,3 \%$ & $2,3 \%$ & $-13 \%$ \\
\hline $\begin{array}{l}\text { Esaote Bio- } \\
\text { medica } \\
\text { Deutsch- } \\
\text { land, Köln, } \\
\text { Deutschland }\end{array}$ & Mylab 50 & 30 & $-19,5 \%$ & $3,1 \%$ & $-13 \%$ \\
\hline \multicolumn{6}{|c|}{$n$ Anzahl der Probanden } \\
\hline
\end{tabular}

lumen-Stitching" sind ein regelmäßiger Herzrhythmus und die Kooperation des Patienten für ein Atemanhaltemanöver während der Exspiration. Zusätzlich gelten bei der Bildoptimierung der 3D-Technik natürlich alle physikalischen Grundregeln, die aus der konventionellen 2D-Echokardiographie bekannt sind, so z.B. die Verbesserung der räumlichen Auflösung aller B-Mode- und Dopplermodalitäten bei höheren Dopplerfrequenzen bei gleichzeitiger Reduktion der Schallpenetration. Die Bildschärfe von 3D-Datensätzen kann durch zusätzliche Nachbearbeitung durch Optimierung des Renderings verbessert werden. Bei guter Bildqualität verbessert die 3D-Echokardiographie die kardiologische Diagnostik morphologischer Befunde (z.B. Herzklappendiagnostik) und funktioneller Analysen (Volumenbestimmungen aller Herzhöhlen). Durch genaue 3D-Volumenanalysen des rechten und linken Ventrikels können in Verbindung mit der Dopplerechokardiographie totale und effektive Schlagvolumina ermittelt werden und damit auch objektiv und reproduzierbar Regurgitationsfraktionen bei Klappeninsuffizienzen ermittelt werden [3]. Natürlich müssen für diese Analysen 3D-Bilddatensätze mit guter Bildqualität vorliegen, um genaue Endokardkonturerkennung und die Abgrenzung des Trabekelwerks zu erkennen.

Eine weitere Herausforderung der 3DTechnologie ist die Navigation innerhalb der 3D-Datensätze, um geeignete Blickwinkel zur Darstellung der kardialen Strukturen zu erhalten. Diese Bearbeitung der 3D-Datensätze sollte ausführlich gelehrt und gelernt werden, damit die jeweils besten Bildeinstellungen beim Monitoring von Interventionen für den interventionellen Kardiologen bzw. Herzchirurgen schnell auch im Live-Modus angeboten werden können.

\section{Die Deformationsbildgebung}

Deformation ist die prozentuale Längenänderung eines Körpers relativ zur Aus- gangslänge. Deformationen können unterschiedlich schnell ablaufen. Im Englischen wird die Deformation als Strain und deren Geschwindigkeit (Deformationsrate) als Strain-Rate bezeichnet. Die myokardiale Deformation kann sowohl mittels Gewebedoppler als auch über eine Nachverfolgung der Bewegung der Myokardtextur, dem sog. Speckle Tracking, analysiert werden. Der Gewebedoppler misst eindimensional in Schallstrahlrichtung und ist damit winkelabhängig. Er erlaubt jedoch schnelle punktuelle Messungen, funktioniert selbst bei eingeschränkter Schallbarkeit und hat eine unübertroffene zeitliche Auflösung. Speckle Tracking kann jede Bewegungsrichtung innerhalb der Schnittebene analysieren. Die Bildanalyse ist etwas aufwendiger, liefert jedoch unmittelbar und häufig (semi)automatisch eine Vielzahl von abgeleiteten Parametern. Strain Imaging ist abhängig von qualitativ guten, artefaktfreien Bildern und hat nur die begrenzte zeitliche Auflösung der zugrunde liegenden Bildsequenzen als Limitation. Eine schlechte Bildqualität mit unklaren Myokarddarstellungen führt zu Unterschätzungen der Deformation.

\section{Die linksventrikuläre Deformations- analyse}

Die Deformation des linken Ventrikels ist komplex und kann vollständig nur mit einer Matrix aus 9 Strain-Vektoren (3 orthogonalen Strains und 6 Shear-Strains) oder 3 Principal-Strain-Komponenten beschrieben werden. Am Herzen wird die basal-apikale Komponente der Verformung als longitudinaler Strain bezeichnet, die Wandverdickung wird durch den radialen Strain beschrieben, und die dritte Komponente ist der zirkumferenzielle Strain. Die Verdrehung des Ventrikels kann durch die Differenz der apikalen und basalen Drehbewegung (Twist in Grad) oder normiert auf den Abstand der beiden Drehebenen als Torsion ( $\mathrm{Grad} / \mathrm{cm}$ ) beschrieben werden. In der Systole sind der longitudinale und zirkumferenzielle Strain negativ, da sich das Myokard in diesen Richtungen verkürzt. Der radiale Strain ist positiv, da die Wände sich verdicken [2, 114].

In der Klinik wird in der Regel der longitudinale Strain mittels Speckle Tracking analysiert. Die Auswerteprogramme ar- 
beiten meist (semi-)automatisch und liefern sowohl segmentale als auch globale Strain-Werte, ggf. als "Nebenprodukt" die Ejektionsfraktion, sowie bei Bedarf eine Vielzahl abgeleiteter Parameter. StrainMessungen, die die gesamte Myokardbreite einbeziehen (mitt-myokardialer Strain) sind besonders robust und weniger empfindlich gegenüber den endokardialen Messungen.

Zeitlicher Bezugs- und damit Nullpunkt der Strainskala ist die Enddiastole. Obwohl die meisten Auswerteprogramme hierzu den EKG-Trigger nutzen, ist zu beachten, dass die Enddiastole über den Mitralklappenschluss definiert ist, der v. a. bei Schenkelblöcken und anderen Pathologien mit abweichender QRS-Morphologie nicht immer mit dem Trigger des EKGs zusammenfällt. Hier ist eine manuelle Korrektur nötig, um korrekte Messwerte zu erhalten [114].

Als globalen Strain bezeichnet man die Verkürzung einer gedachten Linie durch das Myokard von Mitralring zu Mitralring. In der Regel wird der Minimalwert der Strain-Kurve im Herzzyklus angegeben, der normalerweise endsystolisch (bei Aortenklappenschluss) auftritt. Bei großen dyskinetischen (Narben-)Gebieten sollte strikt endsystolisch gemessen werden, da der Kurvennadir deutlich später auftreten kann und dessen Messung zu einer Überschätzung des globalen Strains führen könnte. In der Regel werden globale Strain-Messungen aus allen 3 apikalen Schnittebenen gemittelt.

Der normale globale Strain des linken Ventrikels liegt im Bereich von $-20 \%$. Welche Werte als pathologisch anzusehen sind, hängt leider von der verwendeten Software ab. Die - Tab. 3 gibt hierzu eine Herstellerübersicht. Globale StrainMessungen sind aber nicht zuletzt wegen ihrer hohen Automatisierung sehr gut reproduzierbar. Bei klinischen Verlaufsbeobachtungen können selbst kleine Abweichungen (teilweise unter 2\%) als relevant betrachtet werden, wenn Technik des gleichen Herstellers verwendet wurde. Der globale Strain ist ein sensitiver Parameter. Er fällt frühzeitig ab, selbst wenn die Ejektionsfraktion noch normal ist [61].

Für segmentale Strain-Messungen wird der linke Ventrikel in 6 Segmente pro Schnittbild (18-Segment-Modell) unter- teilt. Die Messungen zeigen eine deutlich stärkere Variabilität als beim globalen Strain. Zahlenwerte sollten daher mit Vorsicht und am besten nur innerhalb des Ventrikels relativ zueinander betrachtet werden. Segmentale Strain-Kurven können eine im Zeitverlauf stark abweichende Form aufweisen. Die Formanalyse der Kurven kann wichtige diagnostische Informationen liefern. Typische Muster ergeben sich bei lokalen Fibrosen z. B. durch ischämische Narben. Hier ist die systolische Verkürzung vermindert, und nach Aortenklappenschluss tritt eine postsystolische Verkürzung auf (s. - Abb. 5a). Bei Stressechokardiographien kann eine während der Untersuchung auftretende postsystolische Verkürzung als Zeichen der Ischämie gewertet werden. Schenkelblockbilder zeigen ebenfalls typische Muster. Wenn Kandidaten für eine kardiale Resynchronisationstherapie eine frühzeitige Verkürzung im Septum zeigen, die bei Beginn der verspäteten lateralen Kontraktion eine Knotung zeigt oder in eine Dehnung umschlägt, sind die Aussichten für einen Therapieerfolg sehr hoch (s. - Abb. 5b). Verschiedene Pathologien können auch typische Strain-Verteilungsmuster zeigen. Diese Muster sind jedoch nicht ausreichend spezifisch und sensitiv, um eine Diagnose zu beweisen. So tritt bei kardialer Amyloidose häufig ein „apical sparing" auf, d.h. apikale Strain-Werte sind bewahrt, während mittventrikuläre und basale Strain-Werte bei geringeren Absolutwerten erniedrigt sind. Hypertrophe Kardiomyopathien zeigen v. a. in den hypertrophen Wandabschnitten niedrige Strain-Werte (s. - Abb. 5c).

\section{Die rechtsventrikuläre Deformationsanalyse}

Der rechtsventrikuläre (RV) Strain kann in allen apikalen Anlotungen mittels Gewebedoppler und Speckle Tracking analysiert werden. Somit kann die anteriore RV-Wand durch ventrale Abkippung der apikal langen Achse, die inferiore RV-Wand durch ventrale Abkippung des apikalen 2-Kammer-Blickes und die freie topographisch medial gelegene RV-Wand im apikalen 4-Kammer-Blick unter Zentrierung des rechten Ventrikels dargestellt werden [2, 3]. In der Regel wird nahezu ausschließlich der RV-Strain der freien RV-Wand im RV-fokussierten 4-Kammer-Blick mittels Speckle Tracking analysiert. Auswerteprogramme der neuesten Generation enthalten häufig spezifische Funktionen für die RV-StrainAnalyse. Sind diese nicht vorhanden, kann die Analysefunktion für den linken Ventrikel „zweckentfremdet“ werden. Durch die manuellen Interaktionen mit der Software führt dies allerdings zu einer höheren Variabilität der Messungen.

Der normale globale Strain der freien RV-Wand liegt im Bereich von $-30 \%$ [2, 3]. Bei der Interpretation des RV-Strains sind die angegebenen weiten Bereiche der Referenzwerte problematisch. Deshalb sollte der RV-Strain immer im anatomischen ( $z$. B. RV-Dilatation) und hämodynamischen Kontext ( $z$. B. bei Volumenbelastung infolge einer Trikuspidalklappeninsuffizienz oder eines Shunt-Vitiums) interpretiert werden [50, 66, 85, 92].

Die Reduktion des RV-Strain kann zur frühen Erkennung einer Chemotherapieinduzierten Kardiotoxizität eingesetzt werden [48] sowie zur Charakterisierung der RV-Dysfunktion bei Rechtsherzinfarzierungen und bei arrhythmogener RV-Kardiomyopathie. Zudem weisen Einschränkungen des RV-Strains auf frühe Stadien einer diastolischen LV-Dysfunktion hin.

\section{Die linksatriale Deformationsanalyse}

Neben der morphologischen LA-Beschreibung ist die Analyse der LA-Funktion aus diagnostischen und prognostischen Gründen sinnvoll. Durch eine LA-Deformationsanalyse kann die LA-Funktion einfacher und genauer beurteilt werden als durch konventionelle volumetrische Messungen. Für eine korrekte Strain-Messung muss das linke Atrium in allen 3 apikalen Schnitten systolisch und diastolisch im Zentrum dargestellt werden, um ein "Foreshortening" zu vermeiden. Zusätzlich sollte der Bildsektor zur Erhöhung der Bildrate auf das linke Atrium begrenzt werden. Einige Hersteller bieten spezifische Software zur LAStrain-Analyse an. Ansonsten kann der LAStrain mit der linksventrikulären SpeckleTracking-Software gemessen werden, was weniger komfortabel und mit einer höheren Variabilität der Messungen verbunden ist. Die Messregion wird von Mitralring zu Mitralring gezeichnet, wobei die Einmün- 

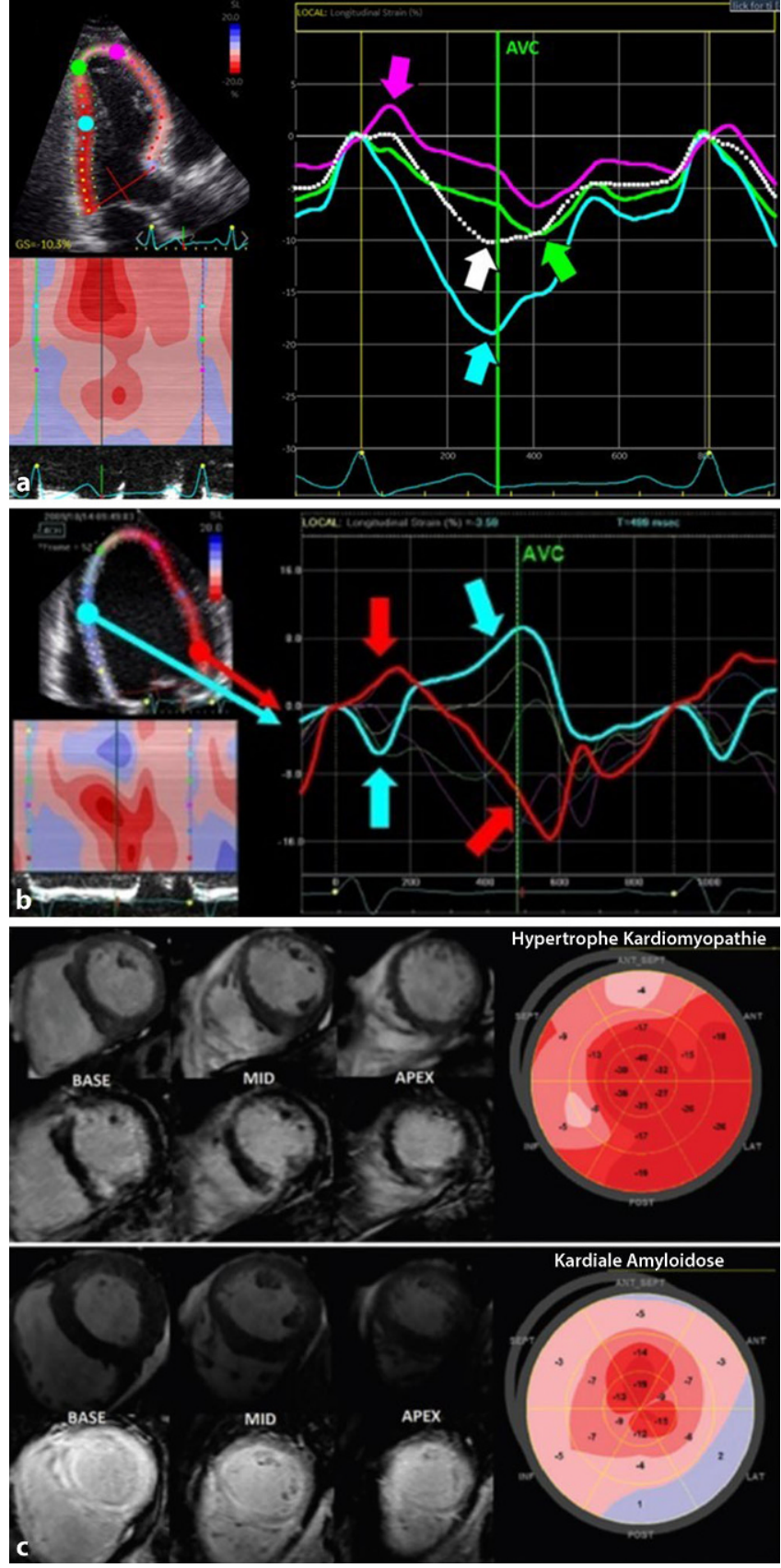

Abb. 5 ॥ Strain bei Infarkt (a). Strain-Analyse am Dreikammerblick eines Patienten nach apikalem Herzinfarkt. Hellblau - normale Strain-Kurve mit systolischem Maximum. Grün - Randbereich des Infarktes mit verminderter systolischer Funktion und geringer postsystolischer Verkürzung. Violett Narbengebiet mit systolischer Dehnung und ausgeprägter postsystolischer Verkürzung. Weiß - globaler Strain. AVC - Aortenklappenschluss. Strain bei Linksschenkelblock (b). Septale (hellblau) und laterale (rot) Strain-Kurven bei einem Patienten mit Linksschenkelblock. Typisch ist die frühe Kontraktion des Septums bei gleichzeitiger Dehnung der lateralen Wand. AnschlieBend kontrahiert die laterale Wand und dehnt das Septum. Dieses Muster entspricht einem guten Ansprechen auf eine Resynchronisationstherapie. Regionale Strain-Verteilungsmuster (c). Oben - Hypertrophe Kardiomyopathie. Der Strain ist im hypertrophierten Septum reduziert. Unten - Kardiale Amyloidose. Typisches "apical sparing“ mit erhaltenen Strain-Werten im linksventrikulären Apexbereich

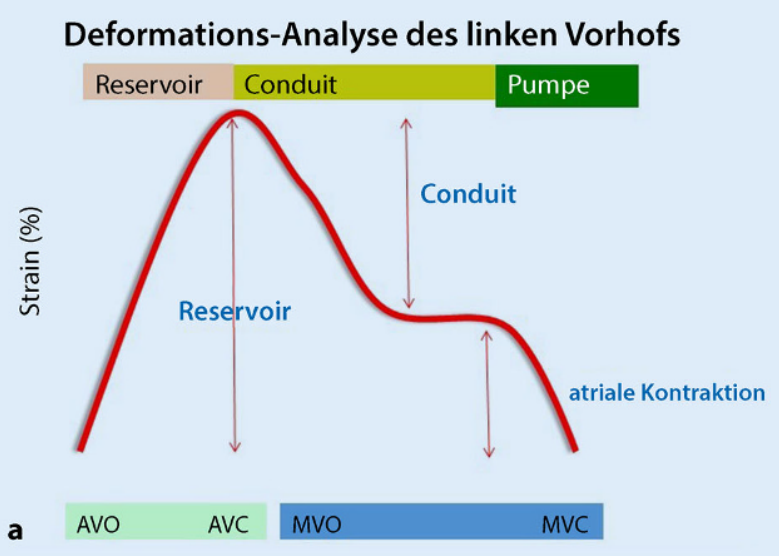

Normalbereiche des LA-Strains und seiner Komponenten: Peak LA-Strain $\approx 40 \%$

\begin{tabular}{|c|c|c|}
\hline LA-Strain-Komponente & Mittelwert (\%) & $95 \%$-Konfidenzintervall \\
\hline Reservoir & 39,4 & $38,0-40,8$ \\
\hline Conduit & 23,0 & $20,7-25,2$ \\
\hline Atriale Kontraktivität & 17,4 & $16,0-19,0$ \\
\hline
\end{tabular}

b

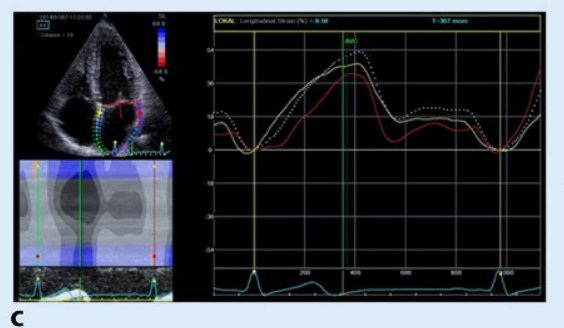

Beispiel:

Normaler

LA-Strain bei

Sinusrhythmus

$=43 \%$

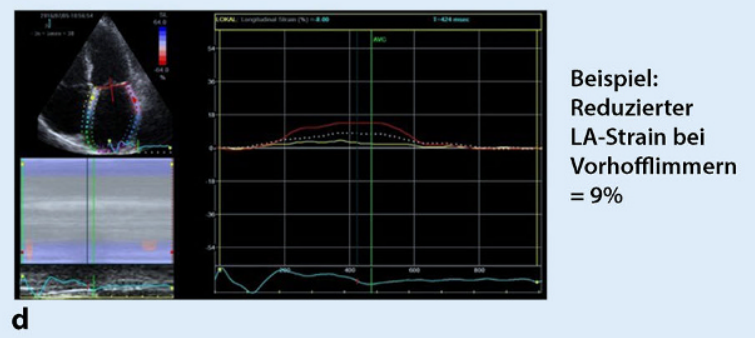

Abb. 6 \ Darstellung der Deformationskurve des LA-Strains während eines Herzzyklus (a); Normalwerte des LA-Strains und seiner Komponenten (b); Beispiel einer LA-Strain-Analyse bei einem normalen Probanden bei normaler LA-Größe und normalen LA-Strain-Werten (c); Beispiel einer LA-StrainAnalyse bei einem Patienten mit paroxysmalem Vorhofflimmern mit LA-Dilatation und hochgradig reduzierten LA-Strain-Werten (d). $A V O_{\text {", aortic valve }}$

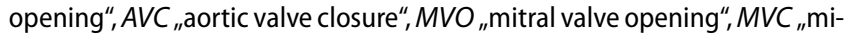
tral valve closure" 
dungen von Vorhofohr und Pulmonalvenen interpoliert werden müssen. Aufgrund der geringen Wanddicke wird am Vorhof nicht zwischen endokardialem und mittmyokardialem Strain unterschieden. Technisch und physiologisch sind segmentale Messungen nicht sinnvoll, sodass atrialer Strain nur global angegeben wird [2, 3, 72]. Zeitreferenz für den Nullpunkt der StrainSkala ist die Enddiastole $[72,86]$.

Die LA-Deformation zeigt bei Sinusrhythmus 3 Phasen. Erstens wird eine LADehnung (Reservoir-Phase) während der LA-Füllung in der LV-Systole beobachtet. In der frühen Diastole folgt die ConduitPhase während des passiven Bluteinstroms in den linken Ventrikel. In der späten Diastole erfolgt die atriale Kontraktion. Diese 3 Komponenten lassen sich aus 2 StrainMessungen endsystolisch und vor Beginn der Kontraktion bestimmen (- Abb. 6). Der LA-Strain bei maximaler Füllung (Reservoir-Funktion) ist derzeit am besten untersucht und beträgt bei Gesunden ca. $40 \%$ [83], wobei jedoch alters- und geschlechtsabhängige Unterschiede bestehen. Der Reservoir-Strain ist auch bei Vorhofflimmern bestimmbar.

Oft zeigt der Reservoir-Strain Einschränkungen der LA-Funktion bereits vor einer LA-Volumen-Zunahme. Daher eignet sich die Messung zur frühen Detektion von diastolischen Funktionsstörungen oder LA-Fibrosierungen. Insbesondere bei Patienten mit klinischen Symptomen einer Herzinsuffizienz bei erhaltener LVEF eignet sich die LA-Reservoir-Funktion - auch nach Rekompensation der Patienten -, das Risiko von Vorhofflimmern und die Gesamtprognose abzuschätzen [44, 47]. Bei Patienten mit Vorhofflimmern wird die ReservoirFunktion des LA-Strains als bester echokardiographischer Surrogatparameter der LA-Fibrosierung beschrieben [20]. Zudem ist ein erniedrigter LA-Strain ein Prädiktor für das Auftreten von Vorhofflimmern sowie bei Schlaganfallpatienten für ein hohes Rezidivrisiko eines Schlaganfalls [19, 62]. In Zusammenhang mit LV-Wandverdickungen ermöglichen LA-Strain-Analysen oft zwischen restriktiven Kardiomyopathien, hypertrophen Kardiomyopathien (ohne Beeinträchtigung des LA-Strains) und kardialen Amyloidosen bzw. Speichererkrankungen (mit Reduktion des LA-Strains) eine Differenzierung [10]. Generell ist je- doch zu berücksichtigen, dass sich die bisherigen Normwerte des LA-Strains je nach Ultraschallhersteller unterscheiden.

Zusammenfassend ist davon auszugehen, dass sich der LA-Strain aufgrund seiner schnellen und einfachen Bestimmung als Routineparameter etablieren wird.

\section{Die Echokardiographie bei angeborenen Herzfehlern im Erwachsenenalter}

Der TTE-Untersuchungsgang bei angeborenen Herzfehlern sollte vor der Dokumentation einer standardisierten TTE-Untersuchung mit der klaren anatomischen Lage des Herzens (Beschreibung des Situs) und der eindeutigen Zuordnung der anatomischen Herzhöhlen beginnen [6, 21, 63]. Daher sollte die TTE bei Patienten mit angeborenen Herzfehlern im Erwachsenenalter mit der subkostalen Anlotung beginnen, um die Lage des Herzens zu den großen Gefäßen und den Organen im Abdomen zu beschreiben. Zur anatomischen Charakterisierung der Ventrikel ist dann z.B. die klare Zuordnung der AV-Klappen notwendig. Die Mitralklappe determiniert den anatomischen linken Ventrikel, die Trikuspidalklappe den anatomisch rechten Ventrikel. Der linke Vorhof ist u.a. durch das linke Vorhofohr, der rechte Vorhof durch die Crista terminalis und die Einmündung des Koronarsinus zu erkennen [6]. In der - Tab. 4 wird eine Auswahl der häufigsten kongenitalen Entitäten im Hinblick auf ihre Anatomie, Begleitanomalien, Veränderung nach therapeutischen Maßnahmen, Komplikationen und deren echokardiographischen Befunde aufgeführt. Bewusst wurde dabei die echokardiographische Beurteilung von Befunden nach Fontan-Operation und totaler cavopulmonaler Anastomose (TCPC) nicht integriert, da sowohl die Ausgangsanatomie als auch die postoperativen Situationen zu verschiedenartig sind, um sie im Rahmen dieser Übersicht tabellarisch darzustellen.

\section{Die Echokardiographie bei und nach kardiochirurgischen Operationen}

Kardiologen, Herzchirurgen und Anästhesisten sollen entsprechend aktuellen Emp- fehlungen $[4,5,49,82]$ in einer Teamentscheidung abwägen, welche interventionelle oder herzchirurgische Therapie im Hinblick auf die jeweilige fachliche Expertise und die lokal verfügbaren Therapieoptionen individuell für den betroffenen Patienten am besten geeignet ist. Grundlagen dieser Teamentscheidungen sind neben der Beurteilung der klinischen Symptomatik und der Komorbiditäten die ätiologische Zuordnung sowie der Schweregrad der jeweiligen kardialen Erkrankungen in Verbindung mit möglichst eindeutig zu charakterisierenden kardialen Funktionsparametern. Neben der Koronarangiographie zur Beurteilung einer begleitenden stenosierenden koronaren Herzerkrankung spielt in diesem Zusammenhang stets die Echokardiographie eine zentrale Rolle.

Vor jeder Herzoperation sollten eine standardisierte Dokumentation - möglichst in gleicher Qualität und mit adäquater technischer Ausstattung - und damit ein vollständig transparenter echokardiographischer Ausgangsbefund als Referenzuntersuchung für spätere postoperative Kontrollen vorliegen [5, 35, 37, 79]. Wegen verschiedener technischer, aber auch morphologischer und funktioneller Aspekte ist es möglich, dass bei diesen Patienten mehrere TTEs und auch TEEs zur unmittelbaren Dokumentation des Ausgangsbefundes bzw. eines Referenzbefundes kurz vor der Operation notwendig sind. Die präoperativen Dokumentationen und Messungen sollten besonders bei Klappenoperationen die Grundlage für eine konkrete herzchirurgische Planung darstellen und zusätzlich eine Risikostratifizierung ermöglichen. Neben dem konventionellen Operationsmodus mit medianer Sternotomie, Anschluss an eine Herz-Lungen-Maschine und Operation in Kardioplegie sind heute auch Operationen am schlagenden Herzen - in der Regel primär für koronare Bypassoperationen - oder Operationen mit unterschiedlichen Zugangswegen wie z.B. über eine sog. Minithorakotomie bzw. videoendoskopische Eingriffe sowie Hybridoperationen zusammen mit Katheterinterventionen als Bestandteile des Standardprogramms einer zeitgemäßen Herzchirurgie anzusehen. 
Tab. 4 Angeborene Herzfehlerim Erwachsenenalter im Hinblick aufdie Anatomie der kongenitalen Veränderung, häufige echokardiographischeSzenarien und ihre Begleitanomalien, Restzustände der Anomalien und Komplikationen nach Korrektur sowie deren Befunddokumentation mit speziellen Tipps und Tricks

\begin{tabular}{|c|c|c|c|}
\hline $\begin{array}{l}\text { Anatomie der kongenitalen Ver- } \\
\text { änderung }\end{array}$ & $\begin{array}{l}\text { Häufige echokardiographische } \\
\text { Szenarien/Begleitanomalien }\end{array}$ & $\begin{array}{l}\text { Restzustände und Komplikati- } \\
\text { onen nach Korrektur }\end{array}$ & $\begin{array}{l}\text { Befunddokumentation } \\
\text { Tipps und Tricks }\end{array}$ \\
\hline $\begin{array}{l}\text { Shuntvitium: ASD (atrialer Sep- } \\
\text { tumdefekt) }\end{array}$ & $\begin{array}{l}\text { Shuntvitium: ASD (atrialer Sep- } \\
\text { tumdefekt) }\end{array}$ & $\begin{array}{l}\text { Shuntvitium: ASD (atrialer Sep- } \\
\text { tumdefekt) }\end{array}$ & $\begin{array}{l}\text { Shuntvitium: ASD (atrialer Sep- } \\
\text { tumdefekt) }\end{array}$ \\
\hline $\begin{array}{l}\text { ASD vom Secundumtyp (ASD II) } \\
\text { ist der häufigster Vorhofseptumde- } \\
\text { fekt (ca. } 80 \% \text { ). Er befindet sich in } \\
\text { der Region der Fossa ovalis } \\
\text { ASD vom Primumtyp (ASD I, } \\
\text { ca. } 15 \% \text { der Vorhofseptumdefekte, } \\
\text { befindet sich in der "Crux cordis“). } \\
\text { Er zählt zu den atrioventrikulären } \\
\text { Septumdefekten (partieller AVSD } \\
\text { oder partieller AV-Kanal) und weist } \\
\text { typischerweise zusätzliche AV- } \\
\text { Klappen-Anomalien auf (s. auch } \\
\text { unter AVSD) } \\
\text { Sinus-venosus-Defekte liegen } \\
\text { im Übergangsbereich des rechten } \\
\text { Vorhofs und der Hohlvenen } \\
\text { - Der obere Sinus-venosus-De- } \\
\text { fekt ist häufiger (ca. } 5 \% \text { der } \\
\text { ASDs) als der untere (<1\%) } \\
\text { und ist typischerweise mit einer } \\
\text { partiellen (manchmal komplet- } \\
\text { ten) Fehlmündung der rechten } \\
\text { Lungenvenen in die obere Hohl- } \\
\text { vene und den rechten Vorhof } \\
\text { vergesellschaftet } \\
\text { - Sinus-venosus-Defekte können } \\
\text { von transthorakal schwierig } \\
\text { darzustellen sein - häufig TEE } \\
\text { notwendig } \\
\text { Der "unroofed“ Koronarsinus ist } \\
\text { eine seltene Form eines ASD mit } \\
\text { einer Verbindung zwischen dem } \\
\text { Koronarsinus und dem linken } \\
\text { Vorhof. Sie kommt fast immer } \\
\text { in Verbindung mit einer linken } \\
\text { oberen Hohlvene vor, die in das } \\
\text { Dach des linken Vorhofs mündet }\end{array}$ & $\begin{array}{l}\text { Rechtsventrikuläre (RV) Volumen- } \\
\text { belastung } \\
\text { Erhöhter pulmonalarterieller Druck } \\
\text { (sPAP) } \\
\text { Sekundäre Trikuspidalklappenin- } \\
\text { suffizienz } \\
\text { Rechtsatriale Dilatation } \\
\text { Lungenvenenfehlmündung (Sinus } \\
\text { venosus und Secundumdefekte) } \\
\text { Persistierende linke obere Hohlve- } \\
\text { ne }\end{array}$ & $\begin{array}{l}\text { Restshunt } \\
\text { Persistierende RV-Dilatation und/ } \\
\text { oder Dysfunktion } \\
\text { Persistierend erhöhter sPAP } \\
\text { Pulmonalvenenobstruktion } \\
\text { Arrosion von Aortenwurzel oder } \\
\text { Vorhofwand durch einen Okkluder } \\
\text { Thrombus im Bereich eines Ver- } \\
\text { schlussdevices } \\
\text { Trikuspidalklappeninsuffizienz }\end{array}$ & $\begin{array}{l}\text { ASD: Lokalisation, Größe, Shun- } \\
\text { trichtung } \\
\text { RV-Größe, RV-Funktion } \\
\text { Pulmonalarterieller Druck (sPAP) } \\
\text { Sekundäre Trikuspidalklappenin- } \\
\text { suffizienz } \\
\text { Begleitanomalien je nach ASD-Typ } \\
\text { Diastolische LV-Funktion } \\
\text { ASD II - vor geplantem perkuta- } \\
\text { nem Verschluss } \\
\text { - Atriale septale Randstrukturen } \\
\text { („rims“). Klärung der Anatomie } \\
\text { des posterioren "rim“ } \\
\text { - Detektion fehlmündender Lun- } \\
\text { genvenen } \\
\text { Nach ASD-Verschluss: } \\
\text { - RV-Größe und -Funktion (Re- } \\
\text { modeling) } \\
\text { - Patch- bzw. Okkluderintegrität } \\
\text { und Restshunt } \\
\text { - Mitral- sowie Trikuspidalklap- } \\
\text { peninsuffizienz } \\
\text { - sPAP } \\
\text { - Diastolische LV-Funktion }\end{array}$ \\
\hline
\end{tabular}




\begin{tabular}{|c|c|c|c|}
\hline $\begin{array}{l}\text { Anatomie der kongenitalen Ver- } \\
\text { änderung }\end{array}$ & $\begin{array}{l}\text { Häufige echokardiographische } \\
\text { Szenarien/Begleitanomalien }\end{array}$ & $\begin{array}{l}\text { Restzustände und Komplikati- } \\
\text { onen nach Korrektur }\end{array}$ & $\begin{array}{l}\text { Befunddokumentation } \\
\text { Tipps und Tricks }\end{array}$ \\
\hline $\begin{array}{l}\text { Shuntvitium: VSD (ventrikulärer } \\
\text { Septumdefekt) }\end{array}$ & $\begin{array}{l}\text { Shuntvitium: VSD (ventrikulärer } \\
\text { Septumdefekt) }\end{array}$ & $\begin{array}{l}\text { Shuntvitium: VSD (ventrikulärer } \\
\text { Septumdefekt) }\end{array}$ & $\begin{array}{l}\text { Shuntvitium: VSD (ventrikulärer } \\
\text { Septumdefekt) }\end{array}$ \\
\hline $\begin{array}{l}\text { Perimembranöse Defekte liegen } \\
\text { im Bereich des membranösen Sep- } \\
\text { tums (ca. } 80 \% \text { ). Sie sind subaortal } \\
\text { und subtrikuspidal gelegen, in der } \\
\text { parasternalen kurzen Achse zwi- } \\
\text { schen } 9 \text { Uhr und } 12 \text { Uhr gelegen } \\
\text { Muskuläre Defekte (ca. 15-20\%) } \\
\text { sind komplett umgeben von ven- } \\
\text { trikulärem Muskelgewebe. Sie sind } \\
\text { im „Inlet-“, im "Outlet-" oder im } \\
\text { apikalen (trabekulären) Anteil des } \\
\text { Septums gelegen } \\
\text { "Doubly committed“-Defekte } \\
\text { („,supracristal“ oder "subarteriell") } \\
\text { sind "Outlet"-Defekte der fibrö- } \\
\text { sen Kontinuität zwischen Aorten- } \\
\text { und Pulmonalklappe. Sie sind } \\
\text { damit direkt angrenzend an die } \\
\text { Semilunarklappen und häufig ver- } \\
\text { gesellschaftet mit einem Prolaps } \\
\text { der rechtskoronaren Tasche der } \\
\text { Aortenklappe mit konsekutiver } \\
\text { Aortenklappeninsuffizienz. Sie } \\
\text { sind in der parasternalen kurzen } \\
\text { Achse zwischen } 12 \text { Uhr und } 3 \text { Uhr } \\
\text { gelegen } \\
\text { Gerbode-Defekte sind eine Dis- } \\
\text { kontinuität des atrioventrikulären } \\
\text { Septums mit resultierendem Shunt } \\
\text { von linkem Ventrikel in den rech- } \\
\text { ten Vorhof. Es gibt angeborene } \\
\text { Entitäten bzw. artifizielle nach } \\
\text { einem AVSD-Verschluss }\end{array}$ & $\begin{array}{l}\text { Linksatriale und linksventrikuläre } \\
\text { Volumenbelastung } \\
\text { Erhöhter pulmonalarterieller Druck } \\
\text { (sPAP) } \\
\text { Prolaps des aortalen Sinus und } \\
\text { Aortenklappeninsuffizienz } \\
\text { Doppelgekammerter rechter Ven- } \\
\text { trikel }\end{array}$ & $\begin{array}{l}\text { Restshunt } \\
\text { Persistierende LV-Dilatation und } \\
\text { systolische oder diastolische Dys- } \\
\text { funktion } \\
\text { Residuell erhöhter pulmonalarteri- } \\
\text { eller Druck (sPAP) } \\
\text { Residuelle Aortenklappenanomalie } \\
\text { und Aortenklappeninsuffizienz } \\
\text { Doppelgekammerter rechter Ven- } \\
\text { trikel } \\
\text { Device-Lokalisation und Interfe- } \\
\text { renz mit umgebenden Strukturen }\end{array}$ & $\begin{array}{l}\text { VSD Lokalisation, Größe, Shun- } \\
\text { trichtung, systolischer Druckgra- } \\
\text { dient; ein diastolischer Fluss > } \\
\text { 1m/s spricht für eine diastolische } \\
\text { Funktionsstörung } \\
\text { LV-Größe und systolische Funktion } \\
\text { LA-Größe } \\
\text { Pulmonalarterieller Druck (sPAP) } \\
\text { Begleitanomalien je nach VSD-Typ } \\
\text { Aortenklappendysfunktion bei } \\
\text { perimembranösem VSD } \\
\text { Bei doppelgekammertem RV } \\
\text { Nachweis eines intraventrikulä- } \\
\text { ren Gradienten } \\
\text { Abschätzung des RV- } \\
\text { Druckes - PRV } \\
\text { VSyst = syst. RR- P } \text { max } \\
\text { Restriktiver VSD= hohe Druck- } \\
\text { differenz zwischen LV und RV bei } \\
\text { geringem Shuntvolumen } \\
\text { VSD nach Verschluss: } \\
\text { - LV-Größe und Funktion (Remod- } \\
\text { eling) } \\
\text { - VSD-Patch- bzw. Okkluderinte- } \\
\text { grität und Restshunt } \\
\text { - Aortenklappeninsuffizienz }\end{array}$ \\
\hline
\end{tabular}




\begin{tabular}{|c|c|c|c|}
\hline $\begin{array}{l}\text { Anatomie der kongenitalen Ver- } \\
\text { änderung }\end{array}$ & $\begin{array}{l}\text { Häufige echokardiographische } \\
\text { Szenarien/Begleitanomalien }\end{array}$ & $\begin{array}{l}\text { Restzustände und Komplikati- } \\
\text { onen nach Korrektur }\end{array}$ & $\begin{array}{l}\text { Befunddokumentation } \\
\text { Tipps und Tricks }\end{array}$ \\
\hline $\begin{array}{l}\text { Shuntvitium: AVSD (atrioventri- } \\
\text { kulärer Septumdefekt) }\end{array}$ & $\begin{array}{l}\text { Shuntvitium: AVSD (atrioventri- } \\
\text { kulärer Septumdefekt) }\end{array}$ & $\begin{array}{l}\text { Shuntvitium: AVSD (atrioventri- } \\
\text { kulärer Septumdefekt) }\end{array}$ & $\begin{array}{l}\text { Shuntvitium: AVSD (atrioventri- } \\
\text { kulärer Septumdefekt) }\end{array}$ \\
\hline $\begin{array}{l}\text { Gemeinsame atrioventrikuläre } \\
\text { Verbindung mit fehlender atrio- } \\
\text { ventrikulärer Septierung } \\
\text { Besonders häufig bei Patienten mit } \\
\text { Down-Syndrom (Prävalenz AVSD } \\
\text { 30\%) } \\
\text { Anatomische Charakteristika sind } \\
\text { - Gemeinsame ovaläre atrioven- } \\
\text { trikuläre Verbindung } \\
\text { - Defekt des membranösen atrio- } \\
\text { ventrikulären Septums } \\
\text { - Eine gemeinsame Klappe mit } \\
\text { 5 Segeln (linkes und rechtes } \\
\text { murales Segel, rechtes ante- } \\
\text { rosuperiores Segel, superiores } \\
\text { und inferiores überbrückendes } \\
\text { Segel) } \\
\text { - Eine "un-wedged“ Aorta mit } \\
\text { elongiertem linksventrikulärem } \\
\text { Ausflusstrakt - LVOT („Gänse- } \\
\text { gurgel-Deformität") } \\
\text { Funktionell liegt entweder partiell } \\
\text { ein Shunt nur auf der Vorhofebene } \\
\text { (sog. ASD vom Primumtyp oder } \\
\text { partieller AVSD) oder komplett ein } \\
\text { Shunt auf atrialer und ventrikulärer } \\
\text { Ebene („common AVSD“) vor } \\
\text { Nach operativer Korrektur bedarf } \\
\text { die atrioventrikuläre Klappendys- } \\
\text { funktion (meist Insuffizienz, selten } \\
\text { Stenose) besonderer Aufmerksam- } \\
\text { keit. Ein Rest-ASD und/oder VSD, } \\
\text { eine LVOT-Obstruktion, eine LV- } \\
\text { und RV-Funktionsstörung und eine } \\
\text { sPAP-Erhöhung müssen ausge- } \\
\text { schlossen bzw. identifiziert werden }\end{array}$ & $\begin{array}{l}\text { Rechtsventrikuläre (RV) Volumen- } \\
\text { belastung } \\
\text { Erhöhter pulmonalarterieller Druck } \\
\text { (sPAP) } \\
\text { Sekundäre Trikuspidalklappenin- } \\
\text { suffizienz } \\
\text { Rechtsatriale Dilatation } \\
\text { Lungenvenenfehlmündung (Sinus } \\
\text { venosus und Secundumdefekte) } \\
\text { Persistierende linke obere Hohlve- } \\
\text { ne } \\
\text { AV-Klappen-Anomalien und LVOT- } \\
\text { Obstruktion } \\
\text { "Double orifice left AV-valve“ } \\
\text { Abnormaler Papillarmuskelverlauf } \\
\text { „Parachute“ linke AV-Klappe } \\
\text { Linksventrikuläre Volumenbelas- } \\
\text { tung } \\
\text { Pulmonale Hypertonie oder Eisen- } \\
\text { menger-Syndrom }\end{array}$ & $\begin{array}{l}\text { Restshunts (atrial und ventrikulär) } \\
\text { RV- und LV-Dilatation und Dys- } \\
\text { funktion } \\
\text { Residuell erhöhter pulmonalarteri- } \\
\text { eller Druck (sPAP) } \\
\text { Linksseitige AV-Klappen-Insuf- } \\
\text { fizienz, die in der Regel durch } \\
\text { einen Schließungsrand zwischen } \\
\text { superiorem und inferiorem über- } \\
\text { brückendem Segel verursacht wird } \\
\text { Rechtsseitige AV-Klappen-Insuffizi- } \\
\text { enz } \\
\text { LVOT-Obstruktion }\end{array}$ & $\begin{array}{l}\text { Kompletter, partieller oder transi- } \\
\text { tionaler AVSD } \\
\text { Größe der atrialen und ventrikulä- } \\
\text { ren Anteile } \\
\text { Shuntrichtung der beiden Anteile } \\
\text { Anatomie der AV-Klappen und } \\
\text { Chordae } \\
\text { AV-Klappen-Insuffizienz } \\
\text { sPAP } \\
\text { Ventrikuläre Größe und Funktion } \\
\text { AVSD nach Korrektur } \\
\text { - Residueller ASD oder VSD } \\
\text { - Residueller LV-RA-Shunt (Gerbo- } \\
\text { de-ähnlich) } \\
\text { - Links- und rechtsseitige AV- } \\
\text { Klappendysfunktion } \\
\text { - Links- und rechtsseitige Ventri- } \\
\text { kelfunktion und -größe } \\
\text { - sPAP } \\
\text { - Vergesellschaftete Anomalien, } \\
\text { V.a. LVOT-Obstruktion }\end{array}$ \\
\hline $\begin{array}{l}\text { Obstruktive Vitien - subvalvuläre } \\
\text { Aortenstenose }\end{array}$ & $\begin{array}{l}\text { Obstruktive Vitien - subvalvuläre } \\
\text { Aortenstenose }\end{array}$ & $\begin{array}{l}\text { Obstruktive Vitien - subvalvuläre } \\
\text { Aortenstenose }\end{array}$ & $\begin{array}{l}\text { Obstruktive Vitien - subvalvuläre } \\
\text { Aortenstenose }\end{array}$ \\
\hline $\begin{array}{l}\text { Subaortale Membran } \\
\text { Fibromuskuläre Leiste } \\
\text { Fibromuskuläre tunnelartige Enge } \\
\text { des linksventrikulären Ausfluss- } \\
\text { traktes (LVOT) } \\
\text { Den LVOT kreuzende Chordae } \\
\text { Basale Septumhypertrophie }\end{array}$ & $\begin{array}{l}\text { Bikuspide Aortenklappe } \\
\text { Aortenisthmusstenose } \\
\text { Supramitraler Ring } \\
\text { „Parachute“-Mitralklappe } \\
\text { Bei Vorhandensein aller dieser } \\
\text { Veränderungen spricht man von } \\
\text { einem „Shone-Komplex“ } \\
\text { Aortenklappeninsuffizienz }\end{array}$ & $\begin{array}{l}\text { Aortenklappeninsuffizienz } \\
\text { LV-Hypertrophie } \\
\text { Systolische und diastolische LV- } \\
\text { Dysfunktion } \\
\text { Pulmonalarterielle Hypertonie }\end{array}$ & $\begin{array}{l}\text { Turbulente Flüsse im LVOT können } \\
\text { zu Alterationen der Taschen der } \\
\text { Aortenklappe mit konsekutiver } \\
\text { Aortenklappeninsuffizienz führen } \\
\text { Das systolisches Flussprofil im } \\
\text { LVOT zeigt eine dynamische Ob- } \\
\text { struktion mit spätsystolischem Ma- } \\
\text { ximum im Gegensatz zur fixierten } \\
\text { Obstruktion mit mittsystolischem } \\
\text { Maximum } \\
\text { Hohe Rezidivrate nach chirurgi- } \\
\text { scher Exzision subaortaler Mem- } \\
\text { branen (bis zu 20\%) } \\
\text { Noch häufiger sind Rezidivraten } \\
\text { bei fibromuskulärer Form der } \\
\text { LVOT-Obstruktion }\end{array}$ \\
\hline
\end{tabular}




\begin{tabular}{|c|c|c|c|}
\hline $\begin{array}{l}\text { Anatomie der kongenitalen Ver- } \\
\text { änderung }\end{array}$ & $\begin{array}{l}\text { Häufige echokardiographische } \\
\text { Szenarien/Begleitanomalien }\end{array}$ & $\begin{array}{l}\text { Restzustände und Komplikati- } \\
\text { onen nach Korrektur }\end{array}$ & $\begin{array}{l}\text { Befunddokumentation } \\
\text { Tipps und Tricks }\end{array}$ \\
\hline $\begin{array}{l}\text { Obstruktive Vitien - valvuläre } \\
\text { Aortenstenose }\end{array}$ & $\begin{array}{l}\text { Obstruktive Vitien - valvuläre } \\
\text { Aortenstenose }\end{array}$ & $\begin{array}{l}\text { Obstruktive Vitien - valvuläre } \\
\text { Aortenstenose }\end{array}$ & $\begin{array}{l}\text { Obstruktive Vitien - valvuläre } \\
\text { Aortenstenose }\end{array}$ \\
\hline $\begin{array}{l}\text { Monocuspide, bicuspide, tricuspi- } \\
\text { de und quadricuspide Klappenan- } \\
\text { lage } \\
\text { Dysplastische Klappenanlagen }\end{array}$ & $\begin{array}{l}\text { Dilatation der Aorta ascendens } \\
\text { Aortenisthmusstenose } \\
\text { Turner-Syndrom }\end{array}$ & $\begin{array}{l}\text { Progrediente Dilatation der Aor- } \\
\text { tenwurzel und Aorta ascendens }\end{array}$ & $\begin{array}{l}\text { Aortenklappeninsuffizienz bei } \\
\text { bicuspider Klappenanlage } \\
\text { Dilatation der Aortenwurzel und } \\
\text { Aorta ascendens } \\
\text { Alle Schallfenster (links paraster- } \\
\text { nale apikale und suprastenale } \\
\text { Anlotung) sind aufgrund der häufi- } \\
\text { gen exzentrischen Jetformationen } \\
\text { zur besten Dokumentation der } \\
\text { transvalvulären Geschwindigkeit } \\
\text { abzuleiten } \\
\text { Über die rechts parasternale An- } \\
\text { lotung in Rechtsseitenlage sollte } \\
\text { die transvalvuläre Geschwindigkeit } \\
\text { am besten mit einer Stiftsonde } \\
\text { abgeleitet werden } \\
\text { Die modifizierte Bernulli-Glei- } \\
\text { chung ist bei LVOT-Geschwindig- } \\
\text { keiten }>1,2 \text { m/s für den prästeno- } \\
\text { tischen Geschwindigkeitswert zu } \\
\text { korrigieren }\end{array}$ \\
\hline $\begin{array}{l}\text { Obstruktive Vitien - supravalvu- } \\
\text { läre Aortenstenose }\end{array}$ & $\begin{array}{l}\text { Obstruktive Vitien - supravalvu- } \\
\text { läre Aortenstenose }\end{array}$ & $\begin{array}{l}\text { Obstruktive Vitien - supravalvu- } \\
\text { läre Aortenstenose }\end{array}$ & $\begin{array}{l}\text { Obstruktive Vitien - supravalvu- } \\
\text { läre Aortenstenose }\end{array}$ \\
\hline $\begin{array}{l}\text { Sanduhrförmige Verengung der } \\
\text { tubulären Aorta ascendens } \\
\text { Hypoplastische Aorta } \\
\text { Verengung des sinutubulären } \\
\text { Übergangs oder der Aorta ascen- } \\
\text { dens } \\
\text { Die Form der Verengung kann } \\
\text { kurzstreckig, langstreckig oder } \\
\text { tunnelartig sein }\end{array}$ & Williams-Syndrom & $\begin{array}{l}\text { Begleitende valvuläre Veränderun- } \\
\text { gen an der Aortenklappe } \\
\text { Sekundäre Veränderungen infolge } \\
\text { der LV-Druckbelastung }\end{array}$ & $\begin{array}{l}\text { Dokumentation der pathologi- } \\
\text { schen Entität - oftmals in nicht } \\
\text { standardisierten Schnittebenen in } \\
\text { der links und rechts parasternalen } \\
\text { sowie apikalen Anlotung } \\
\text { Die Dokumentation der transste- } \\
\text { notischen maximalen Geschwin- } \\
\text { digkeiten erfolgt in der Regel } \\
\text { ebenfalls aus nicht standardisier- } \\
\text { ten Anlotungen } \\
\text { Komplette Dokumentation des } \\
\text { funktionellen Status der Aorten- } \\
\text { klappe sowie der supravalvulären } \\
\text { Stenose }\end{array}$ \\
\hline
\end{tabular}




\begin{tabular}{|c|c|c|c|}
\hline $\begin{array}{l}\text { Anatomie der kongenitalen Ver- } \\
\text { änderung }\end{array}$ & $\begin{array}{l}\text { Häufige echokardiographische } \\
\text { Szenarien/Begleitanomalien }\end{array}$ & $\begin{array}{l}\text { Restzustände und Komplikati- } \\
\text { onen nach Korrektur }\end{array}$ & $\begin{array}{l}\text { Befunddokumentation } \\
\text { Tipps und Tricks }\end{array}$ \\
\hline $\begin{array}{l}\text { Obstruktive Vitien - Aortenisth- } \\
\text { musstenose }\end{array}$ & $\begin{array}{l}\text { Obstruktive Vitien - Aortenisth- } \\
\text { musstenose }\end{array}$ & $\begin{array}{l}\text { Obstruktive Vitien - Aortenisth- } \\
\text { musstenose }\end{array}$ & $\begin{array}{l}\text { Obstruktive Vitien - Aortenisth- } \\
\text { musstenose }\end{array}$ \\
\hline $\begin{array}{l}\text { Die proximale oder supraduk- } \\
\text { tale Aortenisthmusstenose liegt } \\
\text { proximal des Abgangs der linken } \\
\text { A. subclavia } \\
\text { Die distale oder infraduktale Aor- } \\
\text { tenisthmusstenose liegt distal des } \\
\text { Abgangs der linken A. subclavia }\end{array}$ & $\begin{array}{l}\text { Bicuspide Aortenklappe } \\
\text { Gefäßanomalien } \\
\text { Seitendifferenz des Blutdrucks } \\
\text { Blutdruckdifferenz zwischen obe- } \\
\text { ren und unteren Extremitäten }\end{array}$ & "Unklare" LV-Hypertrophie & $\begin{array}{l}\text { Häufig "unerklärte LV-Hypertro- } \\
\text { phie“ } \\
\text { Dokumentation der transstenoti- } \\
\text { schen Geschwindigkeiten mittels } \\
\text { "Continuous wave“(cw)-Doppler in } \\
\text { der Aorta descendens von supras- } \\
\text { ternal - ggf. mit der Stiftsonde } \\
\text { Die Form des cw-Doppler-Signals } \\
\text { ist dabei wichtiger als die Maximal- } \\
\text { geschwindigkeit } \\
\text { Ein diastolischer "run off“ oder } \\
\text { "diastolic tail“ in die Diastole hin- } \\
\text { ein legt die Diagnose einer hoch- } \\
\text { gradigen Stenose mit Kollateral- } \\
\text { kreislauf nahe (normalerweise ist } \\
\text { Aortenfluss mit Ende der T-Welle } \\
\text { im EKG beendet) } \\
\text { Bei längerstreckigen Stenosen ist } \\
\text { der Dopplergradient unzuverlässig, } \\
\text { da die Annahmen für die Bernoulli- } \\
\text { Gleichung nicht mehr gegeben } \\
\text { sind. Zudem kann der maximale } \\
\text { Gradient durch Kollateralen ver- } \\
\text { mindert sein } \\
\text { Eine Verwechslung zwischen Fluss } \\
\text { in der linken Pulmonalarterie und } \\
\text { der Aorta descendens ist möglich } \\
\text { Zur vollständigen Dokumentation } \\
\text { gehört immer ein cw-Doppler- } \\
\text { Spektrum in der Aorta abdominalis } \\
\text { („diastolic run off“) } \\
\text { Bei starker Kollateralenausbildung } \\
\text { kann sich durch moderate Belas- } \\
\text { tung (Herzfrequenz } 90-100 / m i n) \\
\text { die Aufnahmekapazität der Kolla- } \\
\text { teralen erschöpfen und dadurch } \\
\text { den wahren transstenotischen } \\
\text { Gradienten demaskieren }\end{array}$ \\
\hline $\begin{array}{l}\text { Komplexe angeborene Vitien - } \\
\text { korrigierte Fallot-Tetralogie }\end{array}$ & $\begin{array}{l}\text { Komplexe angeborene Vitien - } \\
\text { korrigierte Fallot-Tetralogie }\end{array}$ & $\begin{array}{l}\text { Komplexe angeborene Vitien - } \\
\text { korrigierte Fallot-Tetralogie }\end{array}$ & $\begin{array}{l}\text { Komplexe angeborene Vitien - } \\
\text { korrigierte Fallot-Tetralogie }\end{array}$ \\
\hline $\begin{array}{l}\text { Die korrigierte Fallot-Tetralogie ist } \\
\text { das häufigste zyanotische angebo- } \\
\text { rene Vitium } \\
\text { Obstruktion des rechtsventrikulä- } \\
\text { ren Ausflusstraktes (RVOT) } \\
\text { Subpulmonale infundibuläre Ste- } \\
\text { nose } \\
\text { VSD } \\
\text { Überreitende Aorta } \\
\text { RV-Hypertrophie }\end{array}$ & $\begin{array}{l}\text { ASD in } 33 \% \text { der Fälle = Fallot- } \\
\text { Pentalogie } \\
\text { Rechtsseitig gelegener Aortenbo- } \\
\text { gen } \\
\text { Koronaranomalien in bis } 10 \% \text { der } \\
\text { Fälle } \\
\text { Aortopulmonale oder bronchopul- } \\
\text { monale Kollateralen } \\
22 \text { q } 11-D e l e t i o n \text { in bis } 15 \% \text { der } \\
\text { Fälle } \\
\text { Persistierende linke obere Hohlve- } \\
\text { ne in bis zu } 10 \% \text { der Fälle } \\
\text { AVSD in bis zu } 2 \% \text { der Fälle }\end{array}$ & $\begin{array}{l}\text { Pulmonalklappeninsuffizienz } \\
\text { RVOT-Obstruktion und Pulmonal- } \\
\text { arterienstenose } \\
\text { RV-Dilatation bzw. RV-Dysfunktion } \\
\text { Restriktiver rechter Ventrikel } \\
\text { Rest-VSD/-ASD } \\
\text { Trikuspidalklappeninsuffizienz } \\
\text { Ektasie der Aorta ascendens } \\
\text { Aortenklappeninsuffizienz } \\
\text { LV-Dysfunktion }\end{array}$ & $\begin{array}{l}\text { Neben der kompletten standar- } \\
\text { disierten Dokumentation des LV } \\
\text { müssen speziell RV-Größe und RV- } \\
\text { Funktion analysiert werden } \\
\text { Diastolische RV-Funktion } \\
\text { Pulmonalklappenfunktion } \\
\text { Suche nach Rest-ASDs und Rest- } \\
\text { VSDs } \\
\text { Dokumentation eines VSD-Patch } \\
\text { Weite des Aortenbulbus sowie der } \\
\text { Aorta ascendens } \\
\text { Erhöhung des pulmonalarteriellen } \\
\text { Drucks (sPAP) } \\
\text { Dokumentation potenzieller Ste- } \\
\text { nosen in der rechten und linken } \\
\text { Pulmonalarterie }\end{array}$ \\
\hline
\end{tabular}


Tab. 4 (Fortsetzung)

Anatomie der kongenitalen Veränderung

Komplexe angeborene Vitien Transposition der großen Arterien: Vorhofumkehr bei komplette Transposition der großen Arterien nach Senning (Korrektur mit autogenem Material) oder Mustard (Korrektur mit Perikard oder Dacron-Patches)

Diese Operationen sind eine physiologische, aber keine anatomische Korrektur der atrioventrikulären Konkordanz und ventrikuloarteriellen Diskordanz bei d-Transposition der großen Arterien (= komplette Transposition) Der venöse Blutfluss aus unterer und oberer Hohlvene wird in das linke Atrium umgeleitet und konsekutiv in den linken Ventrikel und die Pulmonalarterie

Der pulmonalvenöse Blutfluss (oxygeniertes Blut) wird in das rechte Atrium umgeleitet und konsekutiv in den rechten Ventrikel und die Aorta

Damit ist der Systemventrikel der anatomisch rechte Ventrikel Mustard und Senning unterscheiden sich chirurgisch, jedoch nicht echokardiographisch
Häufige echokardiographische Szenarien/Begleitanomalien

Komplexe angeborene Vitien Transposition der großen Arterien: Vorhofumkehr bei kompletter Transposition der großen Arterien nach Senning (Korrektur mit autogenem Material) oder Mustard (Korrektur mit Perikard oder Dacron-Patches)

ASDs

Offener Ductus arteriosus

Hämodynamisch nicht relevante VSDs

Hämodynamisch relevante VSDs in bis zu $20 \%$ der Fälle

LVOT-Obstruktion in bis zu $5 \%$ der Fälle

Selten venoatriale Shuntverbindungen

Malalignment-Defekte mit Septumdeviation können mit einer Aortenisthmusstenose, thorakalen Aortenanomalie, Subpulmonalstenose sowie Pulmonalanomalie bis zur Pulmonalisatresie vergesellschaftet sein
Restzustände und Komplikationen nach Korrektur

Komplexe angeborene Vitien -

Transposition der großen Arteri-

en: Vorhofumkehr bei kompletter

Transposition der großen Arterien nach Senning (Korrektur mit autogenem Material) oder Mustard (Korrektur mit Perikard oder Dacron-Patches)

Baffle-Obstruktion

- Baffle-Obstruktion der oberen Hohlvene häufiger nach Mustard-Operation

- Baffle-Obstruktion der Pulmonalvenen häufiger nach Senning-Operation

Baffle-Leckagen

- Kleine Leckagen sind häufig und hämodynamisch unbedeutend

- Allerdings können sie zu kryptogenen hirnembolischen Insulten führen

- Größere Leckagen sind seltener, führen aber ggf. zu relevanten Volumenbelastungen

LVOT-Obstruktion in $33 \%$ der Fälle

- Eine funktionelle Pulmonalstenose schützt in der Regel das pulmonalarterielle Gefäßsystem

- Die LVOT-Obstruktion kann dynamisch oder fixiert sein

Pulmonalarterielle Hypertonie

- Die pulmonalarterielle Hypertonie tritt in bis zu $7 \%$ der Fälle ein - speziell bei Patienten mit früher bestehendem VSD sowie später TGA-Korrektur

- Die pulmonalarterielle Hypertonie kann zur LV-Dilatation und dem Verlust der in der Regel vorliegenden subpulmonalen halbmondförmigen LV-Konfiguration führen

Systemischer rechter Ventrikel (RV)

- Zeichen der RV-Hypertrophie

- Zeichen der RV-Dilatation

- Die systolische RV-Funktion des Systemventrikels verschlechtert sich nahezu immer im zeitlichen Verlauf

Trikuspidalklappeninsuffizienz

- Die Klappeninsuffizienz erfolgt in der Regel durch eine sekundäre Ringdilatation (selten primär)
Befunddokumentation Tipps und Tricks

Komplexe angeborene Vitien Transposition der großen Arterien: Vorhofumkehr bei kompletter Transposition der großen Arterien nach Senning (Korrektur mit autogenem Material) oder Mustard (Korrektur mit Perikard oder Dacron-Patches)

Neben der kompletten standardisierten Dokumentation des LV müssen speziell RV-Größe und RVFunktion analysiert werden. RVGröße und RV-Funktion müssen im Verlauf dokumentiert werden Die Abschätzung des systolischen pulmonalarteriellen Druckes erfolgt über die Mitralklappeninsuffizienz, da der linke Ventrikel den Pulmonalkreislauf speist Der mittlere pulmonalarterielle Druck kann aus dem Regurgitationssignal der Pulmonalklappe abgeleitet werden

Die anatomische Anordnung von Pulmonalklappe und Aortenklappe ist sehr variabel. Die Aortenklappe erkennt man an den Abgängen der Koronarien aus der Aortenwurzel Eine Zunahme des Schweregrades der Trikuspidalklappeninsuffizienz ist ein Zeichen einer progredienten Schwäche des Systemventrikels Turbulenzen im Bereich der Baffle weisen auf Stenosierungen oder Leckagen hin

Bei Verdacht auf Komplikationen an den Baffles ist zur genauen Diagnose eine transösophageale Echokardiographie bei entsprechender Expertise indiziert 
Tab. 4 (Fortsetzung) Anatomie der kongenitalen Veränderung

Komplexe angeborene Vitien Transposition der großen Arterien: partielle operative Korrektur der Anatomie durch RastelliOperation

Physiologische und anatomische zu d-TGA bestehendem VSD

Der Blutfluss durch den linken Ventrikel wird durch einen Patch über VSD in Aorta umgeleitet Die Pulmonalarterien werden proximal abgetrennt und mit einem - in den RV-inserierenden - klappentragenden Conduit verbunden. Dieses Conduit liegt meist anterior und unmittelbar substernal Die Rastelli-Technik wird auch bei mit VSD und Truncus arteriosus eingesetzt operative Korrektur bei zusätzlich "double outlet right ventricle"

Häufige echokardiographische

Komplexe angeborene Vitien Transposition der großen Arterien: arterielle Switch-Operation

Es erfolgt die vollständige physiologische und anatomische Korrektur

Die Aorta, die ursprünglich aus RV entspringt, wird auf ihre korrekte Position versetzt und mit dem LV verbunden. Die morphologische Pulmonalklappe verbleibt allerdings in ihrer Position als "NeoAortenklappe"

Die Koronarien werden in die NeoAortenwurzel reimplantiert Der Pulmonalarterienstamm wird nach anterior vor die Aorta gebracht - das sog. LeCompte-Manöver - und mit der morphologischen Aortenklappe als "NeoPulmonalklappe" verbunden Szenarien/Begleitanomalien

Komplexe angeborene Vitien Transposition der großen Arterien: partielle operative Korrektur der Anatomie durch RastelliOperation

ASDs

Offener Ductus arteriosus

Hämodynamisch nicht relevante

VSDs

Hämodynamisch relevante VSDs in bis zu $20 \%$ der Fälle

LVOT-Obstruktion in bis zu $5 \%$ der Fälle

Selten venoatriale Shuntverbin-

dungen

Malalignment-Defekte mit Septumdeviation können mit einer Aortenisthmusstenose, thorakalen Aortenanomalie, Subpulmonalstenose sowie Pulmonalanomalie bis zur Pulmonalisatresie vergesellschaftet sein

Restzustände und Komplikationen nach Korrektur

Komplexe angeborene Vitien -

Transposition der großen Arteri-

en: partielle operative Korrektur

der Anatomie durch Rastelli-

Operation

LVOT-Obstruktion

Conduit-Dysfunktion zwischen

rechtem Ventrikel und Pulmonalarterien

VSD-Patch-Leck

Aortenwurzeldilatation

LV- und RV-Dysfunktionen

Arrhythmien

Komplexe angeborene Vitien -

Transposition der großen Arte-

rien: arterielle Switch-Operation

ASDs

Offener Ductus arteriosus

Hämodynamisch nicht relevante

VSDs

Hämodynamisch relevante VSDs in bis zu $20 \%$ der Fälle

LVOT-Obstruktion in bis zu $5 \%$ der

Fälle

Selten venoatriale Shuntverbindungen

Malalignment-Defekte mit Septumdeviation können mit einer Aortenisthmusstenose, thorakalen Aortenanomalie, Subpulmonalstenose sowie Pulmonalanomalie bis zur Pulmonalisatresie vergesellschaftet sein

Komplexe angeborene Vitien Transposition der großen Arterien: arterielle Switch-Operation

LV- und RV-Dysfunktionen Supravalvuläre Pulmonalarterienstenose, was zu Re-Interventionen in 5-30\% der Fälle führt

Supravalvuläre Aortenstenose, was seltener in nur bis zu $2 \%$ der Fälle zu Re-Interventionen führt Rechtsventrikuläre Ausflusstraktobstruktion (RVOTO) bei hypertrophem Infundibulum Aortenwurzel kann die Pulmonalarterie komprimieren

Eine Aortenklappeninsuffizienz entwickelt sich in bis zu $50 \%$ der Fälle

Eine systolische LV-Dysfunktion
Befunddokumentation Tipps und Tricks

Komplexe angeborene Vitien Transposition der großen Arterien: vollständige operative Korrektur der Anatomie durch Rastelli-Operation

Dokumentation der Ursprungsanatomie vor Rastelli-Operation (TGA, "double outlet right ventricle" - DORV, Truncus arteriosus communis)

Detektion von VSD-Patch-Leckagen

Detektion von LVOT-Obstruktion

Dokumentation von Größe und

Form der Aortenwurzel sowie deren Veränderungen Detektion eine RV-PA-Obstruktion des Conduits zwischen RV und Pulmonalarterien, in der Regel durch Degeneration mit Kalzifizierung Beurteilung einer Pulmonalklappeninsuffizienz

Abschätzung des systolischen RVDrucks

Eine progressive Dilatation der ist häufig bei Patienten mit Koronaranomalien zu beobachten
Komplexe angeborene Vitien Transposition der großen Arterien: arterielle Switch-Operation

Neben der kompletten standardisierten Dokumentation der LVGröße und LV-Funktion müssen speziell genauso akkurat RV-Größe und RV-Funktion analysiert werden. RV-Größe und RV-Funktion müssen im Verlauf dokumentiert werden

Abschätzung des systolischen RVDrucks

Dokumentation der Durchgängigkeit der Pulmonalarterien und deren Äste, v. a. nach LeCompteManöver

Dokumentation der Aortenklappenmorphologie und Aortenklappenfunktion sowie die genaue zel - insbesondere im Verlauf Analyse der Weite der Aortenwur- 


\begin{tabular}{|c|c|c|c|}
\hline $\begin{array}{l}\text { Anatomie der kongenitalen Ver- } \\
\text { änderung }\end{array}$ & $\begin{array}{l}\text { Häufige echokardiographische } \\
\text { Szenarien/Begleitanomalien }\end{array}$ & $\begin{array}{l}\text { Restzustände und Komplikati- } \\
\text { onen nach Korrektur }\end{array}$ & $\begin{array}{l}\text { Befunddokumentation } \\
\text { Tipps und Tricks }\end{array}$ \\
\hline $\begin{array}{l}\text { Komplexe angeborene Vitien - } \\
\text { natürlich korrigierte Transpositi- } \\
\text { on der großen Arterien }\end{array}$ & $\begin{array}{l}\text { Komplexe angeborene Vitien - } \\
\text { natürlich korrigierte Transpositi- } \\
\text { on der großen Arterien }\end{array}$ & $\begin{array}{l}\text { Komplexe angeborene Vitien - } \\
\text { natürlich korrigierte Transpositi- } \\
\text { on der großen Arterien }\end{array}$ & $\begin{array}{l}\text { Komplexe angeborene Vitien - } \\
\text { natürlich korrigierte Transpositi- } \\
\text { on der großen Arterien }\end{array}$ \\
\hline $\begin{array}{l}\text { Es besteht eine atrioventrikuläre } \\
\text { und ventrikuloarterielle Diskor- } \\
\text { danz („,Doppel-Diskordanz", „I- } \\
\text { TGA“) } \\
\text { Dadurch ist der RV ist der subaor- } \\
\text { tale Ventrikel und entspricht dem } \\
\text { Systemventrikel } \\
\text { Der LV ist der subpulmonale Ven- } \\
\text { trikel und ist dem kleinen Kreislauf } \\
\text { vorgeschaltet } \\
\text { Die systemische AV-Klappe ist } \\
\text { morphologisch die Trikuspidal- } \\
\text { klappe } \\
\text { Es besteht eine „reversed crux } \\
\text { cordis“, was eine Insertion des sep- } \\
\text { talen Trikuspidalsegels (linksseitig } \\
\text { gelegen) distal der Insertion des } \\
\text { anterioren Mitralsegels (rechtssei- } \\
\text { tig gelegen) bedeutet }\end{array}$ & $\begin{array}{l}\text { Dextrokardie } \\
\text { VSDs } \\
\text { Trikuspidalanomalien, z. B. Ebstein- } \\
\text { artige Malformationen } \\
\text { Systemische AV-Klappenanomali- } \\
\text { en } \\
\text { Trikuspidalklappeninsuffizienz } \\
\text { entsprechend einer AV-Klappenin- } \\
\text { suffizienz des Systemventrikels } \\
\text { Aortenklappeninsuffizienz ent- } \\
\text { sprechend einer Taschenklappen- } \\
\text { insuffizienz im Körperkreislauf } \\
\text { RV-Dysfunktion (Systemventrikel) } \\
\text { Subvalvuläre Pulmonalstenose }\end{array}$ & $\begin{array}{l}\text { Die natürlich korrigierte Transpo- } \\
\text { sition der großen Arterien wird in } \\
\text { der Regel konservativ geführt. Da- } \\
\text { her sind LV- und RV-Dysfunktionen } \\
\text { sowie Insuffizienzen der Trikuspi- } \\
\text { dalklappe im Systemventrikel als } \\
\text { Hinweise einer Verschlechterung } \\
\text { des kardialen Zustandes frühzeitig } \\
\text { zu detektieren } \\
\text { Bei Einschränkung der Funktion } \\
\text { des Systemventrikels sind operati- } \\
\text { ve Maßnahmen bis hin zur Implan- } \\
\text { tation von Herzersatzsystemen } \\
\text { sowie der Herztransplantation } \\
\text { individuell zu diskutieren }\end{array}$ & $\begin{array}{l}\text { Neben der kompletten standar- } \\
\text { disierten Dokumentation der LV- } \\
\text { Größe und LV-Funktion müssen } \\
\text { speziell akkurat die RV-Größe und } \\
\text { RV-Funktion (Systemventrikel) im } \\
\text { Zeitverlauf analysiert werden } \\
\text { Analyse der systemischen AV- } \\
\text { Klappenfunktion (anatomisch } \\
\text { Trikuspidalklappe) } \\
\text { Analyse der Aortenklappenmor- } \\
\text { phologie und deren Funktion } \\
\text { Analyse der Morphologie des } \\
\text { rechtsventrikulären Ausflusstraktes } \\
\text { sowie Beurteilung der Hämodyna- } \\
\text { mik des subpulmonalen Ausfluss- } \\
\text { trakts } \\
\text { Dokumentation einer subvalvulä- } \\
\text { ren Pulmonalstenose }\end{array}$ \\
\hline $\begin{array}{l}\text { Ebstein-Anomalie der Trikuspi- } \\
\text { dalklappe }\end{array}$ & $\begin{array}{l}\text { Ebstein-Anomalie der Trikuspi- } \\
\text { dalklappe }\end{array}$ & $\begin{array}{l}\text { Ebstein-Anomalie der Trikuspi- } \\
\text { dalklappe }\end{array}$ & $\begin{array}{l}\text { Ebstein-Anomalie der Trikuspi- } \\
\text { dalklappe }\end{array}$ \\
\hline $\begin{array}{l}\text { Verlagerung des septalen Segels } \\
\text { der Trikuspidalklappe in den rech- } \\
\text { ten Ventrikel. Das posteriore Segel } \\
\text { ist auch bisweilen verlagert. Die } \\
\text { Segel selbst sind oft dysplastisch } \\
\text { mit verdickten und verkürzten } \\
\text { Chordae und dysplastischen Papil- } \\
\text { larmuskeln } \\
\text { Das anteriore Trikuspidalsegel ist } \\
\text { verlängert mit abnormer Chordae- } \\
\text { Insertion } \\
\text { Diese Trikuspidalkonfiguration } \\
\text { führt zu einer „Atrialisation“ der } \\
\text { basalen Anteile des rechten Ven- } \\
\text { trikels mit abnormer Wandbewe- } \\
\text { gung des Ventrikelseptums }\end{array}$ & $\begin{array}{l}\text { ASD oder PFO (häufig bidirektiona- } \\
\text { ler Shunt) } \\
\text { VSD } \\
\text { Pulmonalatresie mit intaktem } \\
\text { Ventrikelseptum } \\
\text { Mitralklappenprolaps } \\
\text { Aortenisthmusstenose } \\
\text { LV-Noncompaction Kardiomyopa- } \\
\text { thie }\end{array}$ & $\begin{array}{l}\text { Die Ebstein-Anomalie wird in der } \\
\text { Regel konservativ geführt. Daher } \\
\text { sind LV- und RV-Dysfunktionen } \\
\text { sowie Insuffizienzen der Trikus- } \\
\text { pidalklappe als Hinweise einer } \\
\text { Verschlechterung des kardialen Zu- } \\
\text { standes frühzeitig zu detektieren }\end{array}$ & $\begin{array}{l}\text { Dokumentation der Trikuspidal- } \\
\text { klappenanatomie unter Berück- } \\
\text { sichtigung der Verlagerung in } \\
\text { Richtung Apex und Rotation in } \\
\text { Richtung zum RVOT } \\
\text { Beurteilung der Größe und Funkti- } \\
\text { on des funktionellen RV bzw. des } \\
\text { RVOT } \\
\text { Beschreibung des Schweregrades } \\
\text { der „Atrialisation“ des RV } \\
\text { Charakterisierung des Schweregra- } \\
\text { des der Trikuspidalklappeninsuffi- } \\
\text { zienz } \\
\text { Dokumentation der Weite der } \\
\text { Pulmonalarterien } \\
\text { Dokumentation Größe und Funk- } \\
\text { tion des LV im Rahmen einer stan- } \\
\text { dardisierten Dokumentation } \\
\text { Detektion begleitender Kommu- } \\
\text { nikationsdefekte (PFO, ASD, VSD) }\end{array}$ \\
\hline
\end{tabular}


Intraoperativ wird die TEE - derzeit möglichst die 3D-TEE - regelhaft bei Klappenoperationen, in zunehmender Zahl aber auch bei allen anderen Herzoperationen eingesetzt. Intra- und postoperative Befunde sollten für spätere Vergleiche in einheitlicher standardisierter Form aufgezeichnet und in einem zentralen Archiv gespeichert werden. $\mathrm{Zu}$ den prinzipiellen Aufgaben der intraoperativen TEE [80] gehören a) die Kontrolle bzw. Bestätigung der präoperativen Befunde insbesondere Herzklappenpathologien, b) die Detektion von zuvor nicht diagnostizierten Befunden, c) das Monitoring bei der Positionierung von Kanülen für die Herz-Lungen-Maschine, d) die Kontrolle der zentralen Hämodynamik unter pharmakologischer und Volumentherapie bzw. unter der Beatmung, e) die Kontrolle der Entlüftung der Herzkammern beim Abgang von der Herz-Lungen-Maschine, f) die Kontrolle des operativen Ergebnisses an rekonstruierten bzw. prothetisch versorgten Herzklappen bei Wiederherstellung des kardiopulmonalen Kreislaufs, g) die Detektion von morphologischen bzw. funktionellen kardialen Veränderungen direkt nach der Operation, die sofortige Korrekturmaßnahmen erfordern können, wie z.B. neu auftretende Wandbewegungsstörungen (als Hinweis auf Koronararterien- oder Bypassverschlüsse), Zunahme von Klappeninsuffizienzen, funktionelle Klappenstenosen, Perikardergüsse, iatrogene Aortendissektionen oder andere Gefäßverletzungen, und h) die Überprüfung einer suffizienten elektromechanischen Kopplung bei erforderlicher Schrittmacherstimulation [80].

Die Eröffnung des Thorax und der direkte operative Eingriff am Herzen führen zu einer physiologischen Imbalance mit intra- und extravasalen Flüssigkeitsverschiebungen. Blutverlust, erniedrigter peripherer Widerstand beim Wiederaufwärmen, unspezifische Entzündungsreaktionen im Zusammenhang mit der Perfusion über die Herz-Lungen-Maschine und erhöhter Vorlastbedarf bei einer durch die Operation bedingten Compliancestörung infolge eines unterschiedlich ausgeprägten postischämischen myokardialen Stunnings erzeugen in der Regel einen hohen Volumenbedarf [106]. Die während der Operation mittels TEE gesteuerte dif- ferenzierte medikamentöse Therapie (u.a. Inotropika und Vasopressoren) und die Volumenjustierung zur Optimierung der Vor- und Nachlast sowie der Auswurfleistung unter gleichzeitiger maschineller Beatmung sollte möglichst ohne Unterbrechung auf der Intensivstation fortgesetzt werden. Das hämodynamische Monitoring über invasiv und/oder peripher gemessene Drücke sowie Blutgasanalysen [93] kann durch differenzierte echokardiographische Untersuchungen sinnvoll ergänzt werden. In Abhängigkeit von den operativen Zugangswegen sind oft unmittelbar postoperativ auch mittels TTE relevante hämodynamische Befunde abzuleiten. In der frühen postoperativen Phase ist bei Fragestellungen zu klappenspezifischen Details oft eine TEE indiziert. Eine TEE kann auch aufgrund oft eingeschränkter postoperativer TTE-Untersuchungsbedingungen notwendig sein.

Bei jeder Herzoperation können auch bei unkompliziertem Verlauf hämodynamische Veränderungen gegenüber den - in der Regel durch eine TTE dokumentierten - präoperativen Vorbefunden auftreten. Aus diesem Grund ist es wichtig, die Füllungsvolumina und die globale Pumpfunktion der Herzkammern echokardiographisch qualitativ und quantitativ zwischen prä- und postoperativem Status vergleichen zu können. Als Beispiel soll die postoperative echokardiographische Kontrolle der hämodynamischen Auswirkung unter Medikation bei Patienten mit präoperativ dokumentierten hypertrophierten sowie hyperkinetischen linken Ventrikeln mit kleinem Cavum sein. Falls postoperativ ein niedriger peripherer arterieller Druck vorliegt, ist pathophysiologisch einer Volumengabe sowie einer negativ chronotropen Medikation Vorrang vor Medikamenten mit positiv inotroper, chronotroper sowie vasopressorischer Wirkung zu geben. Positive therapeutische Effekte können schnell echokardiographisch verifiziert werden.

LVEF-Werte sowie LV-Strain-Analysen sind früh postoperativ oft wegen des Stunnings des Myokards nach den erfolgten Manipulationen weniger aussagefähig als präoperativ.

Eine präoperativ reduzierte RV-Funktion hat eine ungünstige Prognose in Bezug auf den frühen und langfristigen Ver- lauf nach Herzoperationen [85]. Postoperativ nach kardiochirurgischen Manipulationen treten jedoch generell Einschränkungen der RV-Funktion auf, die durch signifikante Reduktion der TAPSE und des RVStrains zu dokumentieren sind. Die dreidimensional berechnete RV-Ejektionsfraktion sowie Parameter wie die "Fractional Area Change" zeigen dagegen keine signifikanten Veränderungen [74]. Aufgrund der Komplexität der Beurteilung der postoperativen RV-Funktion sollten alle echokardiographisch zu bestimmenden Parameter der RV-Funktion für die Therapiesteuerung genutzt werden [69].

Das effektive LV- und RV-Schlagvolumen sowie das Herzminutenvolumen bzw. deren Veränderungen im Verlauf sind wichtige Parameter des postoperativen Verlaufs, die trotz bekannter Fehlermöglichkeiten bei den echokardiographischen Kalkulationen zuverlässig bestimmt werden können. Zum Vergleich prä- und postoperativer Befunde sollten möglichst robuste Messmethoden verwendet werden wie z.B. bei unauffälligem LVOT oder gut einsehbaren Dimensionen der Pulmonalklappe dopplerechokardiographische Messungen der Schlagvolumina. Unter postoperativen eingeschränkten Untersuchungsbedingungen zeigen planimetrisch oder volumetrisch bestimmte Schlagvolumina oft hohe Schwankungen. Diastolische Funktionsparameter sowie Abschätzungen des systolischen pulmonalarteriellen Drucks sind postoperativ unmittelbare Indikatoren für die Füllungsdrücke des Herzens. Die Detektion von postoperativen Pleuraergüssen (möglichst im Sitzen und semiquantitativ zu bestimmen) sowie Perikardergüssen ergänzt die kardiale Funktionsdiagnostik [10], da insbesondere akute therapeutische Maßnahmen davon abhängen. Im Falle einer therapierefraktären Herzinsuffizienz muss individuell unter Berücksichtigung echokardiographischer Befunde der Einsatz einer mechanischen Kreislaufunterstützung bzw. einer extrakorporalen Oxygenierung in die Behandlung einbezogen werden.

Spezielle Anforderungen an die Echokardiographie bestehen besonders bei Herzklappenerkrankungen. Bei der AS können in der Regel Informationen zur subvalvulären LV-Morphologie sowie zur Aortenwurzel nicht ausreichend durch 
eine TTE beantwortet werden, sodass nahezu immer eine TEE notwendig ist. Die TEE bei AS sollte klare Aussagen zu Verkalkungen des Aortenringes und des Septum aorticomitrale, zu Kalkeinlagerungen des hinteren Mitralrings, zu einer begleitenden MS-Komponente, zu Dimensionen des Aortenrings und der Aortenwurzel sowie zur Ventrikel- und subaortalen Muskelwulstkonfiguration ermöglichen. Diese Befunde beeinflussen die Operationsplanung im Hinblick auf mechanische Entkalkungen über den Aortenring hinaus bis auf das vordere Mitralsegel oder eine subaortale Myektomie. Obwohl oft die zu implantierende Prothesengröße anhand der Aortenringgröße abzuschätzen ist, führen kleine Klappenringe $<21 \mathrm{~mm}$ Durchmesser zu Überlegungen im Hinblick auf die chirurgische Planung, wie z. B. den zu wählenden Prothesentyp und den Implantationsmodus (intra- vs. supraanulär) oder eine zusätzliche Aortenplastik. Zusätzliche operative Eingriffe an der Aortenwurzel erfordern in der Regel eine längere Operationszeit und haben je nach Operationstechnik unterschiedliche Risiken, wie z.B. AV-Leitungsblockierungen bis hin zu seltenen Ventrikelperforationen. Weiterhin kann die Ausrichtung der zentralen Achse der Prothese anhand einer 3D-Darstellung der Aortenbasis geplant werden [32, 33, 105, 106], wobei eine nichtkoaxiale Orientierung postoperativ mit deutlich höheren transprothetischen Flussgeschwindigkeiten und Gradienten verbunden ist. Bei uni- oder bikuspider AS liegen häufig asymmetrische Konstellationen der Aortenwurzel vor, die durch eine 3D-TEE exakt für die Operationsplanung diagnostiziert werden können. Da diese Informationen Risikokonstellationen beschreiben, die sowohl für den chirurgischen wie auch interventionellen Klappenersatz wichtig sind [16, 52, 82, 83], ist im Hinblick auf eine operative Planung die AS häufig nicht ausreichend durch eine alleinige TTE diagnostiziert. Prä- und postoperativ sollen bei der AS die gleichen echokardiographischen Parameter bestimmt werden. Für Kalkulationen mit der Kontinuitätsgleichung sollte postoperativ anstelle des LVOTDurchmessers der innere Diameter des proximalen Prothesenringes verwendet werden. Bei Prothesen in Aortenpositi- on soll der Parameter des auf die Körperoberfläche bezogenen PPM bestimmt werden. PPM-Werte $>0,85 \mathrm{~cm}^{2} / \mathrm{m}^{2}$ werden als prognostisch unbedenklich, zwischen 0,65 und $0,85 \mathrm{~cm}^{2} / \mathrm{m}^{2}$ als mittelgradig und $<0,65 \mathrm{~cm}^{2} / \mathrm{m}^{2}$ als hochgradig belastend eingestuft [62]. Weitere postoperative Herausforderungen bei AS-Patienten sind die klare Detektion paravalvulärer Lecks und ihre Schweregradquantifizierung sowie Beeinträchtigungen der Mitralklappenfunktion durch Veränderungen der LVOTGeometrie [59].

Bei Al-Patienten - insbesondere bei jüngeren Patienten - müssen durch die Echokardiographie die Möglichkeiten einer klappenerhaltenden Chirurgie geklärt werden. Dazu werden spezielle Anforderungen an die Charakterisierung der Morphologie der einzelnen Segel sowie die Ausmessung der Dimensionen von Aortenwurzel und aszendierender Aorta benötigt [32, 33]. Auch ohne Rekonstruktionsmöglichkeiten ist durch die Echokardiographie abzuleiten, ob neben einer prothetischen Klappenversorgung der Ersatz der Aortenwurzel und der aszendierenden Aorta oder ggf. nur ein suprakoronarer Aortenklappenersatz indiziert ist. Postoperativ sind neben den bei der AS beschriebenen Aspekten bei Aortenwurzelrekonstruktionen die Abgänge der reimplantierten Koronararterien sowie die Anastomosen der implantierten Aorten prothesen zu kontrollieren.

Bei MI-Patienten sollte präoperativ eine exakte Beurteilung der Morphologie und Funktion der Klappe sowohl in der TTE als auch in der TEE erfolgen. Der Mitralklappenring und die einzelnen Segelabschnitte werden in standardisierten Schnittebenen sowie im 3-D-Datensatz dargestellt und vermessen. $\mathrm{Zu}$ den Basisparametern gehören die Anulusdurchmesser in der langen Achse und im kommissuralen Schnitt, die Koaptationshöhe sowie die "Tenting Area" und die Länge des vorderen Mitralsegels. Zusätzlich sollten zur Vermeidung postoperativer dynamischer SAM-Obstruktionen (SAM - „systolic anterior movement ${ }^{\prime \prime}$ ) die Abstände der zentralen Koaptationslinie zum interventrikulären Septum sowie der Winkel zwischen LVOT-Achse und Aortenbasis vermessen werden $[46,68,80]$. Weiterhin kann durch die echokardiographischen
Befunde auf die Rekonstruktionsfähigkeit einer Klappe durch Beschreibung von Verkalkungen und der Konsistenz des Segelmaterials geschlossen werden. Da Prothesen in Mitralposition prinzipiell in den funktionellen nativen Mitralklappenring implantiert werden, spielt die präoperative echokardiographische Beurteilung des Mitralringes bei allen MI- und auch MS-Patienten eine wesentliche Rolle. Intraoperativ muss bei Mitralklappenrekonstruktionen das Operationsergebnis nach Wiederaufnahme der kardialen Kreislauffunktion auf Dichtigkeit und eine funktionelle Stenosierung überprüft werden. Wegen der Lage des Ramus circumflexus im Sulcus transversalis am hinteren Mitralring muss bei Mitralklappenoperationen eine iatrogene Koronarokklusion speziell durch neue posterolaterale Wandbewegungsstörungen - ausgeschlossen werden. Da postoperativ bei erfolgreicher Operation einer hochgradigen $\mathrm{MI}$ die LVEF abnimmt, ist im Vergleich zum präoperativen Befund die Aufrechterhaltung des Vorwärtsschlagvolumens bzw. Herzzeitvolumens repräsentativ und prognostisch relevant [44, 47]. Grundsätzlich umfasst die postoperative Echokardiographie nach Mitralklappenrekonstruktion bzw. Mitralklappenersatz die vollständige Dokumentation aller kardialen Funktionsparameter - insbesondere die Messung der transvalvulären Gradienten sowie die Detektion möglicher Leckagen [81, 113]. Bei auffälligen Werten sollten immer Klappen- bzw. Prothesenthrombosen durch eine TEE beurteilt werden $[59,64]$.

\section{Die Echokardiographie beim Monitoring von Interventionen bei strukturellen Herzerkrankungen}

Aufgrund vieler Spezifikationen erfordert das echokardiographische Monitoring von Katheterinterventionen mittels TEE eine gezielte und intensive Ausbildung [42]. Die Qualität der Bildgebung bei der Intervention entscheidet oft über die Minimierung von möglichen Komplikationen und den Behandlungserfolg. Neben der notwendigen Präsentation der kardialen Strukturen vor und während der Interventionsmaßnahmen für die interventionell tätigen Kardiologen sind die Sequenzen der Interventionsabfolge sowie das 
Interventionsergebnis in adäquater Weise zu dokumentieren (d.h. multiplane Echokardiographie, spektral und farbkodierte Dopplerechokardiographie, konventionelle und farbkodierte 3-D-Datensätze etc.). Die Durchführenden des echokardiographischen Monitorings müssen in der Lage sein, Bildsequenzen schnell zu akquirieren und zu dokumentieren, insbesondere bei interventionellen Manövern, bei denen ein Risiko besteht, kardiale Strukturen zu beschädigen.

Zusätzlich zu den spezifischen technischen Aufgaben beim Interventionsmonitoring mittels TEE müssen für den echokardiographisch im Katheterlabor oder Hybridoperationssaal Tätigen ein optimaler Strahlenschutz und ein ergonomischer Arbeitsplatz gewährleistet sein. Die Echokardiographieeinheit sollte am Kopfende des Kathetermessplatzes positioniert werden. Mindestens eine bodentiefe Strahlenschutzwand muss den echokardiographischen Arbeitsplatz komplett vor Streustrahlung abschirmen [17]. Die TEE-Sonde wird generell um die Strahlenschutzwand herumgeführt. Der Handgriff der Sonde sollte auf einem zusätzlichen Tisch abgelegt werden können, um Bewegungsartefakte zu vermeiden. Im Fall einer gegenüber der Linksseitenlage stark eingeschränkten Anlotungsqualität in strenger Rückenlage kann ein Anheben der linken Schulter die Schallbedingungen verbessern. Zusätzlich bestehen besondere Anforderungen an die Patientensicherheit und deren Komfort, da u. a. lange Untersuchungszeiten berücksichtigt werden müssen. Kürzere Eingriffe wie der Verschluss von Vorhofseptumdefekten oder des LAAs sind daher problemlos in tiefer Sedierung möglich $[86,118]$. Dagegen ist bei einer Eingriffsdauer von $>30-60$ min oder bei Interventionen, die von reduzierten Atemexkursionen profitieren, eine Intubationsnarkose angemessen $[119,121]$.Im Folgenden werden v. a. praktische Aspekte des TEEMonitorings bei Interventionen an strukturellen Herzerkrankungen dargelegt.
Die perkutane Mitralklappentherapie (Edge-to-Edge-Therapie - Anuloplastie - perkutaner Klappenersatz)

Die Aufgabe des TEE-Monitorings bei perkutanen Eingriffen an der Mitralklappe ist einerseits die exakte Darstellung der Mitralklappe und ihrer Pathologie sowie andererseits die Visualisierung der DeviceKatheter und ihrer Interaktion mit dem Herzklappengewebe [102, 117]. Die einzelnen prozeduralen Schritte erfordern spezifische echokardiographische Einstellungen (- Abb. 7). Zu Beginn jeder Intervention sollte eine aktuelle Analyse der Klappenpathologie unter Sedierung stehen [121]. Die biplane Anlotung der Mitralklappe mit einem interkommissuralen Schnitt als Primärebene ist dafür zu bevorzugen, da mit dieser Einstellung die Segelbeschaffenheit entlang der gesamten Koaptationslänge gut kontrolliert werden kann. Für kommissurnahe Pathologien muss die Primärebene angepasst werden, um die perpendikuläre biplane Ebene senkrecht zur Koaptation einzustellen. Bei medialer Klappenpathologie erfolgt die Einstellung der primären Schallebene in Richtung 4-Kammer-Blick - „Verringerung der Gradzahl“; bei lateraler Pathologie in Richtung lange Achse - „Zunahme der Gradzahl“. Wichtige, akut zu erhebende Messparameter sind Segellänge, Anulusdiameter, Koaptationstiefe, Prolapsgröße, Flailbreite und Flailhöhe. Alternativ können diese Parameter in der multiplanen Rekonstruktion eines 3D-TEE-Datensatzes bestimmt werden. Die Planimetrie der Mitralklappenöffnungsfläche kann ebenfalls im konventionellen oder farbkodierten 3-D-Datensatz (bevorzugt sind gezoomte 3D-Datensätze, um eine möglichst hohe räumliche und zeitliche Auflösung zu erzielen) vorgenommen werden, wobei ein zusätzliches 3-D-Rendering der Mitralklappe unter Zuhilfenahme variabler Beleuchtungseffekte hilfreich ist, um einen Überblick über das Ausmaß und die Lokalisation der wesentlichen Pathologie zu erhalten.

Zu Beginn der Intervention ist die korrekte Position der transseptalen Punktionsstelle für die optimale Kathetersteuerung entscheidend [112]. Für die Segeltherapie wird eine möglichst weit posteriore Punktion in der Fossa ovalis an- gestrebt. Diese wird im midösophagealen Kurzachsenblick kontrolliert. Das "Tenting“ des interatrialen Septums durch die Punktionsnadel vor der Punktion soll möglichst weit zur atrialen Hinterwand liegen, da ein ausreichend posteriorer Punktionsabstand zur anterioren Position der Mitralklappe in Bezug zur Fossa ovalis für die Intervention notwendig ist. Diese Lokalisation wird anatomisch irreführend in der Literatur als sog. „Punktionshöhe" beschrieben und wird klassischerweise im 4-KammerBlick gemessen. Häufig liegt jedoch das Tenting der Punktionsnadel nicht in der Schnittebene des 4-Kammer-Blicks. Alternativ kann dann in einem modifizierten gespiegelten 4-Kammer-Blick (Einstellung beiGradzahl um $160^{\circ}$ ) die Höhe des Tenting direkt oder mittels biplaner Technik indirekt dargestellt werden. In stark dilatierten Vorhöfen muss eine zu "hohe" Punktion vermieden werden. Bei medialen Mitralklappenpathologien kann eine posteriore und zugleich besonders inferiore Punktion für die Interventionsdurchführung hilfreich sein.

Das Ansteuern der Mitralklappe mit dem Device-tragenden Katheter sollte in einem groß gewählten 3D-Zoom-Volumen erfolgen. Im Idealfall kann die gesamte Vorhoflänge in das Volumen integriert werden und so die Kathetersteuerung auch im hinteren Teil des Vorhofs - insbesondere im Bereich der lateralen Pulmonalvenenostien und der Umschlagsfalte zum LAA visualisiert werden. Als Primärebene für die Generierung des 3D-Volumens bietet sich die interkommissurale Schnittebene an. Für die finale Ausrichtung des Katheters im Bereich der Zielregion empfiehlt sich erneut die Schnittebenen-korrigierte biplane Anlotung. Sie ermöglicht, den Ansteuerungswinkel des Device-tragenden Katheters zeitgleich in 2 Ebenen zu kontrollieren. Die Ausrichtung der Device-Arme in Bezug auf die Mitralklappe kann einfach in einem 3D-Volumen dargestellt werden. Bei guter Bildqualität kann zeitgleich die Funktionstüchtigkeit der Fangelemente überprüft werden. Um Auslöschungsartefakte des Devices durch den Device-tragenden Katheter zu vermeiden, sind häufig geringe Veränderungen der Sondenposition mit zusätzlichem Vorschub in den tieferen Ösophagus notwendig. 

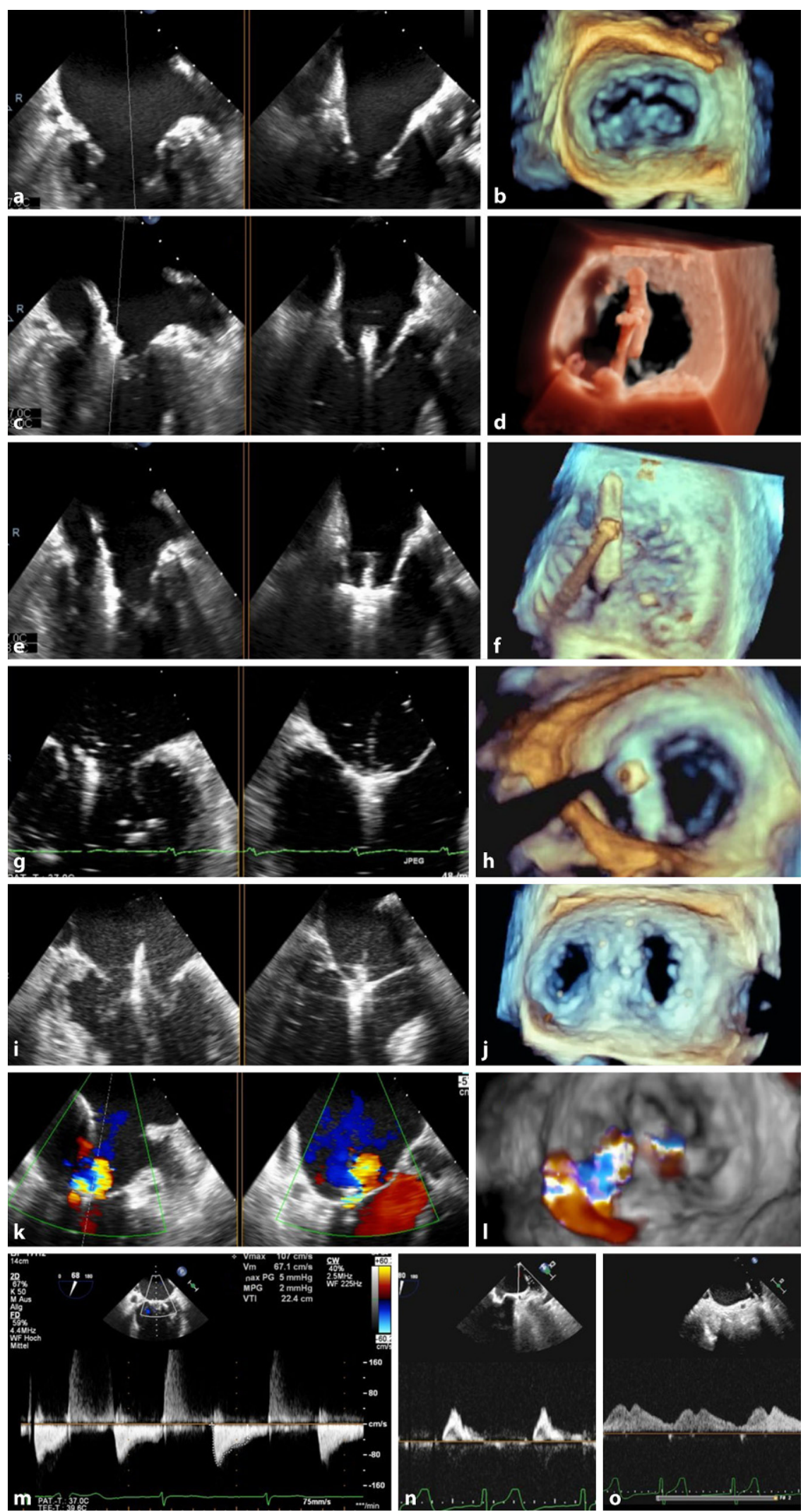

Abb. $7 \triangleleft$ Echokardiographische periinterventionelle TEE-Dokumentation bei der Edge-to-Edge-Repair-Segeltherapie der Mitralklappe. Biplane Darstellung mit der Primärebene des kommissuralen Blickes der Mitralklappe mit perpendikulärer Darstellung der zentralen Segelbereiche (a); zugehöriger 3D-en-face-Blick auf die Mitralklappe vom linken Atrium aus (b); Ausrichtung des Devices in biplaner Darstellung oberhalb der Mitralklappenebene (c), zugehöriger En-face-Blick mit geöffnetem Device (d); Aufladen beider Segelränder in biplaner Darstellung (e); zugehörige 3D-Kontrolle der Ausrichtung des Devices senkrecht zu der Koaptationslinie (f); Herabsenken der Greifarme des Devices in biplaner Darstellung sowie Fassen beider Segelanteile durch das Device (g); zugehörige 3D-en-face-Kontrolle der Device-Orientierung (h); Device-Schluss in biplaner Darstellung (i); Dokumentation der "Brückenformation" durch das Device mit Ausbildung eines "Double Orifice" in der En-face-3D-Darstellung (j); farbkodierte biplane Kontrolle der Restleckagen nach Fassen beider Segel (k); multidimensionale Darstellung der Restleckagen in einem farbkodierten 3D-Datensatz (I); Dokumentation der transvalvulären Blutflussgeschwindigkeiten im Continuous-wave-Doppler nach Device-Implantation ( $\mathbf{m}$ ) sowie der Blutflussgeschwindigkeiten in der linken Lungenvene vor (n) und nach Device-Implantation (o). TEE transösophageale Echokardiographie 
Um ein optimales Reparaturergebnis zu erzielen, muss die Visualisierung der Interaktion von Device und Segeln möglichst exakt und artefaktfrei erfolgen. In Analogie zur Klappenanalyse hat sich auch für diesen Teil der Prozedur die biplane Anlotung als effektiv erwiesen. Sie bietet den Vorteil, dass Bewegungen des Katheters in mediolateraler Ausrichtung mit dem Trackball der Ultraschalleinheit "verfolgt" werden können und so die Längsachsendarstellung elektronisch und nicht durch Manipulation der TEE-Sondenposition stabil gehalten werden kann. Wichtig ist hierbei, die Primärebene so zu wählen, dass die sekundäre Ebene immer exakt parallel zur korrekten Längsausrichtung des Devices verläuft. Bei Pathologien medial oder lateral der A2/P2-Segmente muss hierfür die Primärebene entsprechend der Einstellung zur primären Klappenanalyse angepasst werden. Die korrekte Ausrichtung des Devices sollte zusätzlich in einem aus der Primärebene heraus generierten 3DVolumen im Ventrikel noch einmal bestätigt werden. Zur besseren Visualisierung der Device-Arme kann die Verstärkung des Ultraschallsignals verringert werden. Das Fangen der Segel sowie das Verschließen des Devices müssen unter optimalen Schallbedingungen erfolgen. Der gesamte Vorgang sollte als lange Bildschleife (ca. 10 bis 20 Herzaktionen) aufgezeichnet werden. Ein atembedingtes Herausbewegen des Devices aus der Schallebene kann durch Mikrobewegungen der Sondenrotation und -flexion korrigiert werden.

Vor Ablösen des Devices erfolgt die systematische Evaluation des Akutergebnisses. Das Ausmaß der Segelinsertion sollte durch die vollständige Dokumentation des Greif- und Schließmanövers offensichtlich sein. Bei Unsicherheit über die Insertion eines oder beider Segel kann die Gewebebrücke in biplaner Technik durch einen Schwenk in der zur Primärebene perpendikulären Ebene über die Kommissur durch die Device-Region evaluiert werden. Für eine ausreichende Segelinsertion sprechen straff von atrial in das Device hineinziehende Segel, die sich ungefähr um die Armlänge des genutzten Devices verkürzt darstellen. Erscheint die Segelinsertion suffizient, ist der nächste Schritt die Ergebniskontrolle mittels farbkodierter Echokardiographie.
Es gilt, die Größe, die Richtung und den Ursprung der farbkodiert darstellbaren Restturbulenzen zu ermitteln. Kleine, zentrale Jets sprechen indirekt auch für eine suffiziente Segelinsertion. Jets, die unmittelbar aus dem Device kommen, oder neue exzentrische Jets sprechen für einen insuffizienten Device-Schluss bzw. für ein Verziehen oder ein Aufziehen der Klappensegel. Die exakte Quantifizierung der Restinsuffizienz ist schwierig, da die geometrischen Annahmen der gängigen Quantifizierungsmethoden (V. contracta, effektive Regurgitationsöffnung nach Doppler- und PISA-Methode) durch die veränderte Klappenmorphologie und die veränderten Untersuchungsbedingungen unter Sedierung nicht valide sind. Einzig für die intraprozedurale Planimetrie der Regurgitationsfläche im farbkodierten 3DDatensatz ist eine klinische Validierung anzunehmen [1, 7, 64, 120, 121]. Indirekte Hinweise auf den Schweregrad des residuellen Reflux geben die Größe der verbleibenden PISA-Zonen, die Densität des Refluxsignals im Gewebedoppler sowie Änderungen des Pulmonalvenenflusses. Zuletzt muss die Öffnungsfläche der Mitralklappe - am besten ebenfalls in einem 3D-Datensatz - quantifiziert werden. Als Surrogatlösung wird häufig der mittlere transmitrale Gradient bestimmt, der unter $5 \mathrm{mmHg}$ liegen soll [7], obwohl bekannt ist, dass die Messung des transmitralen Dopplergradienten nach interventioneller Mitralklappensprengung keine zuverlässigen Aussagen über das Interventionsergebnis darstellt. Hierbei ist speziell darauf hinzuweisen, dass bei Bradykardie der transmitrale Gradient „falsch“ niedrig sein kann und eine große Differenz zwischen Maximal- und Mittelgradient auf ein noch bestehendes relevantes Regurgitationsvolumen hinweist.

Bei der perkutanen Mitralklappenanuloplastie werden indirekte und direkte Verfahren unterschieden. Bei der indirekten Anuloplastie wird über den in der Nähe zum Mitralring gelegenen Koronarsinus ein Device implantiert, welches ein positives Remodelling des Mitralanulus bewirken kann [27, 29]. Beim direkten Verfahren wird von atrial ein Textilband mit einzelnen Ankern in den Mitralanulus eingeschraubt, das über einen integrierten Mechanismus verkürzt werden kann [71].
Ein TEE-Monitoring der indirekten Anuloplastie ist nicht zwingend notwendig. Bei schwieriger Sondierung des Koronarsinus kann eine Darstellung des Koronarostiums im sog. bicavalen Blick hilfreich sein. Alle weiteren Schritte werden fluoroskopisch gesteuert. Allerdings können die regionale Wandbewegungsanalyse des linken Ventrikels sowie eine Kompression von Koronarabschnitten in den posterolateralen LV-Segmenten echokardiographisch in der TEE erfasst werden [30]. Die akute Erfolgskontrolle der indirekten Anuloplastie durch die TEE hat jedoch keinen Einfluss auf die Prozedur.

Die direkte Anuloplastie ist ohne eine echokardiographische Steuerung nicht durchführbar [27]. Neben der TEE ist auch eine intrakardiale Echokardiographie möglich. Aktuell ist die 3D-TEE die Standardmodalität. Die wesentliche Aufgabe der periprozeduralen Bildgebung ist die Visualisierung des Implantationskatheters. Sein Ansteuerungswinkel und die Positionierung der Katheterspitze im Bereich der Zielregion ermöglichen eine korrekte Implantation des Ankers. Die 3D-Zoom-Funktion bietet sich für die Visualisierung der groben Kathetersteuerung an. Die genaue Positionierung der Katheterspitze in der perianulären Zielregion kann mittels biplaner Technik erfolgen. Alternativ hat sich die Darstellung von Katheterspitze und Anulus mittels multiplanarer Rekonstruktion (MPR) eines 3D-Volumens in Echtzeit bewährt. Mit diesem Verfahren kann die Schnittebene des Anulus optimal zur sicheren Detektion des Abstandes zwischen Zielposition und angrenzenden Strukturen (Mitralsegelaufhängung, Ramus circumflexus und/oder Koronarsinus) eingestellt werden. Zusätzlich vermeidet die Schnittebenenanpassung innerhalb des 3D-Volumens potenziell komplikationsträchtige TEE-Sondenbewegungen. Wichtig ist auch, die Position der Anker echokardiographisch zu kontrollieren. Im Bereich des posteromedialen Anulus kann die Bildgebung durch Verschattungsartefakte des Implantationskatheters erschwert sein.

Insgesamt stellt die direkte Anuloplastie sehr hohe Anforderungen an das TEEMonitoring. Neben einem ergonomischen Arbeitsplatz ist ein differenziertes präprozedurales Training Grundvoraussetzung für erfolgreiche Interventionen. 
Der perkutane Mitralklappenersatz kann mittels ausgewiesener Prothesen auf transseptalem oder transapikalem Wege oder als sog. "Valve-in-Ring"- oder "Valvein-Valve"-Verfahren mit TAVI-Prothesen (TAVI - „transcatheter aortic valve implantation") erfolgen [67, 106]. Primäres Ziel der echokardiographischen Steuerung ist es, die optimale Positionierung der Prothese zu ermöglichen. Hierzu gehört auch die Kontrolle des korrekten Zugangs (transseptale Punktion vs. transapikaler Zugang). Die Prozedurplanung erfolgt mittels kardialer Computertomographie. Die relevanten Parameter Anulusdiameter, Ventrikelgröße, Länge des vorderen Mitralsegels, grobe Abschätzung des sog. Neo-LVOT können aber auch mittels TEE zu Beginn der Prozedur erneut evaluiert werden. Neben der biplanen Darstellung der Mitralklappe hat sich die MPR eines 3D-Zoom-Volumens als sehr hilfreich und effizient erwiesen. Problematisch ist die Messung des anteroposterioren Durchmessers des Mitralanulus, da die Basis des vorderen Mitralsegels im aortomitralen Übergang nicht eindeutig definiert werden kann und so echokardiographische Orientierungspunkte ebenfalls nicht eindeutig definiert sind. Neben der Ausmessung der anulären Durchmesser im zentralen Schnittpunkt der maximalen Längs- und Querausdehnung in einem 3D-Datensatz besteht eine systematische Messoption, den Anulus anterior bis zur Basis der akoronaren Tasche der Aortenklappe zu messen. Alternativ kann der Anulus auch anterior durch eine intertrigonale Hilfslinie definiert werden. Zusätzlich müssen Device-spezifische Messungen berücksichtigt werden.

Unabhängig von der Größe des statischen in der Computertomographie gemessenen Neo-LVOT kann es nach Prothesenimplantation durch eine Verlagerung des vorderen Mitralsegels nach anterior zu einer dynamischen LVOT-Obstruktion kommen. Eine wichtige Aufgabe des TEE-Monitorings ist es, dieses Risiko abzuschätzen. Ein langes vorderes Mitralsegel, das diastolisch in seiner gesamten Breite Kontakt zum Ventrikelseptum hat, muss als Warnzeichen interpretiert werden. Nach Prothesenimplantation sind daher die Flussgeschwindigkeiten im
LVOT - normalerweise vom transgastralen Schallfenster aus - zu dokumentieren.

Während der Implantation hat sich in Analogie zur Segeltherapie der Mitralklappe für die Device-Steuerung die multidimensionale Darstellung bewährt. Die exakte Positionierung der Prothese erfolgt dagegen in biplaner Darstellung mit der primären Schnittebene des interkommissuralen Blicks. Paravalvuläre bzw. paraanuläre Leckagen sollten ebenfalls in biplaner Technik - einschließlich Farbdopplerechokardiographie - untersucht werden. Zur Objektivierung von Regurgitationen dient die farbkodierte multidimensionale Darstellung, wobei sehr Vorhofwand-nahe Jets bei ungünstigen Einstellungen und bei Schattenartefakten des Prothesenrings übersehen werden können.

Die perkutane Trikuspidalklappentherapie (Trikuspidalsegeltherapie Anuloplastie)

\section{Die Segeltherapie der Trikuspidalklappe} entspricht mit Ausnahme der fehlenden transseptalen Punktion dem Behandlungskonzept der Edge-to-edge-Therapie der Mitralklappe [40]. Die technische Durchführung ist aber aufgrund der für die TEE anatomisch ungünstigen, anterioren Position der Trikuspidalklappe anspruchsvoll und erfordert die Kenntnis der optimalen TEE-Schallfenster für die Trikuspidalklappe. Die transösophageale Schallbarkeit kann bei ca. 50\% der Patienten durch ein Vorschieben der TEE-Sonde aus der „klassischen", midtransösophagealen Position in ein tieftransösophageales Fenster deutlich verbessert werden. Je dilatierter der rechte Vorhof (und entsprechend größer die Kontaktfläche mit dem Ösophagus), umso häufiger ist eine wirksame Anlotung aus dem tieftransösophagealen Fenster notwendig. Typischerweise muss die primäre Schallebene der TEE-Sonde für einen korrekten interkommissuralen Blick im tieftransösophagealen Fenster gegenüber der midösophagealen Position von der Gradzahl her erhöht werden. Von großer Bedeutung für ein erfolgreiches TEE-Monitoring ist die Qualität der transgastralen Anlotung der Trikuspidalklappe. In der der transgastralen kurzen Achse können regelhaft alle Segel und Kommissuren der Trikuspidalklappe in einer Ebene dargestellt werden.
Die Informationen über die Klappenmorphologie, die Device-Positionen sowie die Interaktion von Device und Klappensegeln sind in dieser Einstellung gut zu gewinnen. Bei Aufsuchen der korrekten transgastrischen Anlotung kann die fluoroskopische Kontrolle der TEE-Sondenposition hilfreich sein (-Abb. 8).

$\mathrm{Zu}$ Beginn jeder Intervention steht die erneute Analyse der aktuellen Klappenpathologie. Diese erfolgt einmal von transösophageal mittels biplaner Technik aus einem interkommissuralen Blick und zum anderen aus der transgastrischen, kurzen Achse. Bei der biplanen Anlotung hilft wie bei der Mitralklappe ein "Sweep" entlang der Koaptationslinie zwischen septalem Segel und anterioren sowie posterioren Segeln, um Koaptation, Segellängen und Zügelung im anterioren, zentralen und posterioren Bereich der Trikuspidalklappe zu beurteilen. In der transgastralen Anlotung muss darauf geachtet werden, die Trikuspidalklappe exakt transversal anzuloten, um alle Kommissuren unverkürzt darzustellen. Dieser Anlotungswinkel kann mittels biplaner Darstellung kontrolliert werden. Denn bei optimaler transversaler Anlotung der transgastrischen Primärebene wird der Trikuspidalklappenanulus in der Sekundärebene exakt vertikal geschnitten.

Für das korrekte Ansteuern der Trikuspidalklappe muss der Device-tragende Katheter zunächst aus der oberen Hohlvene zurückgezogen und nach anterior gedreht werden. Dieser Schritt wird regelhaft in einem bicavalen Blick begleitet. Die Beugung des Device-tragenden Katheters nach anterior und seine korrekte Ausrichtung im Bereich der Zielregion werden über die biplane Darstellung der Trikuspidalklappe aus einem interkommissuralen 2-Kammer-Blick gesteuert. Die genaue Position des Device-tragenden Katheters in der Klappenebene, die Ausrichtung der Device-Arme und die Interaktionen von Device und Segeln werden transgastrisch gesteuert. Wichtig ist die konsequente Visualisierung der echoreichen Segelränder, um in der Zielregion die Device-Interaktion mit den Segeln und das Greifen der Segel durch das Device sicher zu erkennen. In der sekundären biplanen Längsachsendarstellung werden die Translationsbewegung des Devices und die Ausrichtung der jewei- 

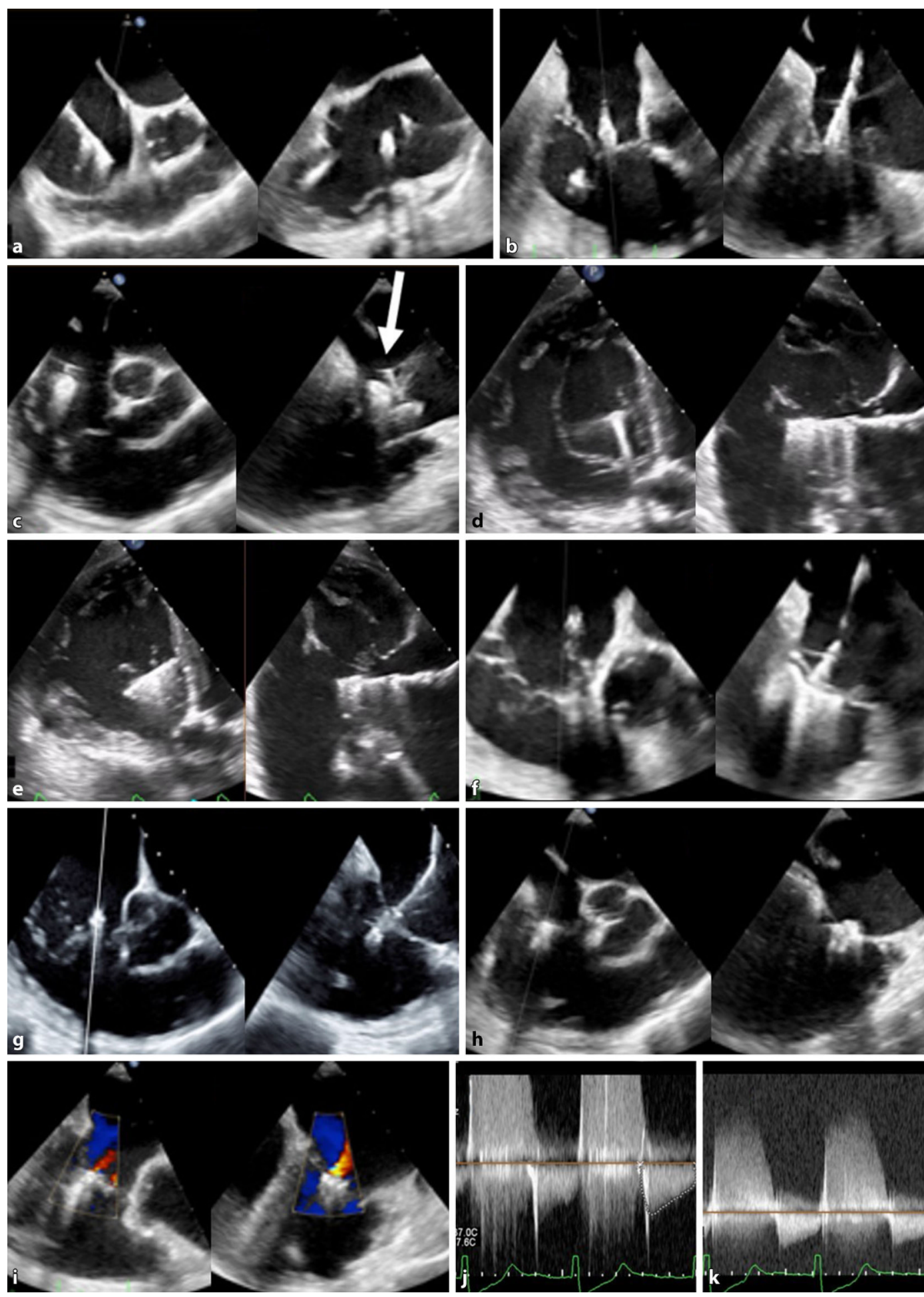

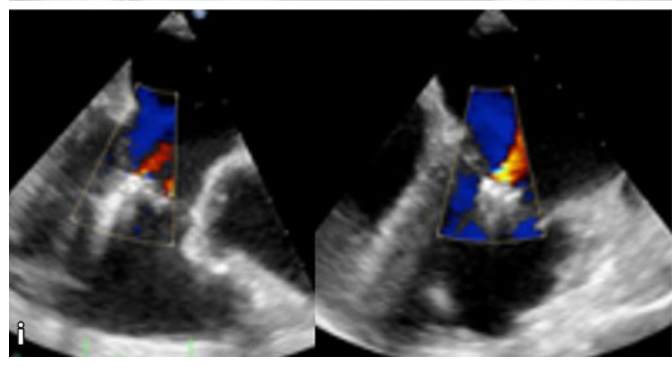

ligen Device-Arme kontrolliert. Das Greifen der Segel wird meist über die transgastrische Anlotung dokumentiert, da in transösophagealer Anlotung eine ausreichende Darstellung der Segel durch katheterassoziierte Schallartefakte verhindert wird. Das korrekte Greifen der Segel ist am sichersten durch eine optimale Darstellung des Greif- vorgangs zu belegen. Um Schallartefakte in der transgastrischen Anlotung durch bereits implantierte Devices zu verhindern, empfiehlt sich bei mehreren Devices die Implantation von anterior nach posterior. Die Erfolgskontrolle erfolgt in Analogie zur Segeltherapie der Mitralklappe. Die korrekte Segelinsertion in das Device soll-
Abb. $8<$ Echokardiographische periinterventionelle TEE-Dokumentation bei der Edge-to-Edge-RepairSegeltherapie der Trikuspidalklappe. Alle Bildsequenzen sind in biplaner Technik aufgenommen. Ansteuern des Device-tragenden Katheters zur Trikuspidalklappe mit Darstellung der Einfluss-Ausfluss-Ebene des rechten Ventrikels als Primärebene aus mittiger ösophagealer Anlotung (a); Adjustierung der Darstellung in tiefösophagealer Anlotung (b); Ausrichten des Devices senkrecht zur zu behandelnden Kommissur sowie Überprüfung der Device-Funktion vor Eintauchen in den rechten Ventrikel (c); transgastraler Blick auf die kurze Achse der Trikuspidalklappe mit Darstellung aller $3 \mathrm{Se}$ gelränder (d); Ausrichtung des Devices und Visualisierung der Segelinteraktionen in der transgastralen Darstellung (e); Aufladen der jeweiligen Segelanteile der Trikuspidalklappe auf das Device in midösophagealer Anlotung (f); Dokumentation Segelinsertion in der tiefen ösophagealen Anschallung vor (g) und nach Ablösen des Devices (h); farbkodierte Kontrolle der Restleckagen nach Intervention (i); Dokumentation des Blutflussgeschwindigkeiten des transtrikuspidalen Blutflusses vor (j) und nach der Intervention (k). TEE transösophageale Echokardiographie 
wendig ist, und - je weiter posteroseptal die Device-Position gelegen ist - eine höhere Einstellung der Gradzahl notwendig ist. Die Beurteilung von Restinsuffizienzen sowie der verbleibenden Klappenöffnungsfläche erfolgt entsprechend den Techniken bei der Segeltherapie der Mitralklappe (semiquantitativ über das Farbdopplersignal und die V.-contracta-Fläche im 3D-Datensatz, Ableitungen der transvalvulären Flussgeschwindigkeiten). Eine vollständige Abdichtung der Trikuspidalklappe durch eine Segeltherapie ist technisch in der Regel nicht möglich. Wichtig ist zu berücksichtigen, dass nach DeviceImplantation ein geringerer transvalvulärer Gradient als in Mitralposition akzeptiert werden darf.

Die perkutane Trikuspidalklappenanuloplastie entspricht vom Konzept exakt dem direkten Anuloplastieverfahren für die Mitralklappe [78]. Dazu wird ein Textilband von der antero- bis zur posteroseptalen Kommissur in den lateralen Anulus implantiert, welches nach abgeschlossener Implantation aktiv verkürzt wird. Die interventionelle Bildgebung erfordert insbesondere die Visualisierung des Implantationskatheters und dessen Spitze im Bereich der Zielregion einschließlich der angrenzenden kardialen Strukturen (Segelaufhängung, Verlauf der rechten Koronararterie, Mündung des Koronarvenensinus). Neben der biplanen transösophagealen Anlotung hat sich die Visualisierung des Implantationskatheters mittels Echtzeit-MPR in einem 3D-ZoomVolumen bewährt. Wichtig hierfür ist eine artefaktfreie Generierung des 3D-ZoomVolumens. Die größte Herausforderung für das TEE-Monitoring ist die Visualisierung des Implantationskatheters im Bereich des posterioren Trikuspidalanulus, da einerseits durch den Katheter selbst, aber auch durch die Nähe zur posterioren Vorhofwand starke Auslöschungsartefakte entstehen können. Eine Möglichkeit, dieses Phänomen zu kompensieren, ist die 2D-Anlotung des Anulus aus einem transgastrischen Fenster. Es ist anzunehmen, dass diese Probleme in Zukunft durch die intrakardiale Echokardiographie - insbesondere wenn auch mit dieser Methode 3D-Darstellungen zum Einsatz kommen minimiert werden können.
Der kathetergestützte Aortenklappenersatz (TAVI)

Der kathetergestützte Aortenklappenersatz ist nicht auf eine periinterventionelle TEE angewiesen. Er kann sehr sicher unter rein fluoroskopischer Kontrolle durchgeführt werden [28]. Die Rolle der periinterventionellen TEE beschränkt sich deswegen auf das Komplikationsmanagement (Fehlpositionierung, paravalvuläre Insuffizienz, Perikarderguss, Anulusruptur) [8, 39]. Grundsätzlich kann ein TEE-Monitoring alle prozeduralen Schritte begleiten (Sondierung linker Ventrikel, Positionierung Prothese, Implantation), doch der eingeschränkte zusätzliche Informationsgewinn wiegt die Nachteile einer für die TAVI sonst nicht notwendigen Intubation bzw. tiefen Sedierung nicht auf. Je nach Strahlengang kann die TEE-Sonde sogar die angiographische Darstellung der Aortenwurzel bei der Klappenimplantation beeinträchtigen. Für die Planung der Prozedur hat sich die kardiale Computertomographie etabliert. Prinzipiell kann aber auch die TEE - insbesondere die 3D-TEE - die Dimensionen des Aortenklappenrings und der Aortenwurzel sowie die Lokalisation der Koronarostien genau vermessen [32, 33, 91], was bei Notfallindikationen oder Kontraindikationen für eine Computertomographie eine Rolle spielen kann. Eine Limitation der Echokardiographie ist oft die Beeinträchtigung der Klappendarstellung durch Kalkschatten.

Die häufigste Aufgabe einer periinterventionellen TEE ist die Evaluation einer paravalvulären Protheseninsuffizienz. Neben den konventionellen Methoden zur Graduierung einer nativen valvulären Aorteninsuffizienz $[89,121]$ kann versucht werden, die zirkumferenzielle Ausdehnung der paravalvulären Regurgitationen um den Klappenstent darzustellen. Hierbei müssen die Anzahl der Refluxjets, ihre Breite unmittelbar am Jetursprung, ihr Verlauf und die Darstellung möglicher Flusskonvergenzzonen berücksichtigt werden [121]. Neben der kurzen Achse der Aortenklappe eignet sich in der TEE die biplane Anlotung mit der langen Achse als Primärachse. Mittels eines Schwenks der kurzen Achse als Sekundärebene entlang des Klappenstents können auf diese Weise die Ursprungsebenen der paraprotheti- schen Regurgitationen ermittelt werden $[120,121]$. Bei optimalen Schallbedingungen kann die Farbdopplerinformation auch in einem 3-D-Farbdopplervolumen analysiert werden (Planimetrie der 3-DVena contracta). Eine transgastrische Anlotung ermöglicht oft die Ableitung der Regurgitationsgeschwindigkeiten.

Am Ende jeder TAVI-Prozedur und bei unklarer hämodynamischer Instabilität muss mittels Echokardiographie ein Perikarderguss ausgeschlossen werden. Die Ätiologie eines Perikardergusses ist echokardiographisch insbesondere von transthorakal nur schwer zu eruieren. Eine Ventrikelperforation ist in der Notfallsituation sicher nur selten zu identifizieren. Die Differenzialdiagnose einer Anulusruptur kann wesentlich sensitiver in der TEE erfasst werden. Auch wenn sie primär fluoroskopisch gestellt wird, ist die TEE für die Beschreibung von Position und Ausmaß der Ruptur sowie potenzieller Shuntvitien unabdingbar. Nach der Prozedur müssen die Gradienten über der Prothese dokumentiert werden. So können postinterventionelle Beschleunigungen der transprothetischen Geschwindigkeiten im Verlauf (z.B. bei Prothesenthrombose) interpretiert werden. Zudem muss eine dynamische LVOT-Obstruktion nach Nachlastsenkung durch die TAVI (engl. „suicide ventricle") ausgeschlossen werden.

\section{Der interventionelle Verschluss des} linken Vorhofohres (LAAO)

Ein TEE-Monitoring - insbesondere durch 3D-TEE (biplane Darstellung, MPR von 3DVolumina) - liefert wichtige Informationen beim interventionellen Verschluss des linken Vorhofohres (• Abb. 9). Die Durchführung erfordert allerdings eine ausreichend tiefe Sedierung des Patienten, um in Rückenlage die TEE-Sonde für die Eingriffszeit (ca. 20-40 min) zu tolerieren. Eine Intubationsnarkose kann im Einzelfall sinnvoll sein, ihr regelhafter Einsatz ist aber nicht zu empfehlen. Zur Sicherung der Atemwege kann ein Guedel- oder Wendel-Tubus hilfreich sein, der problemlos neben der TEE-Sonde platziert werden kann. Ein stabiles Monitoring von Sauerstoffsättigung und Hämodynamik sowie das Vorhalten eines Beatmungsbeutels sind wichtige Voraussetzungen für eine sichere LAAO oh- 

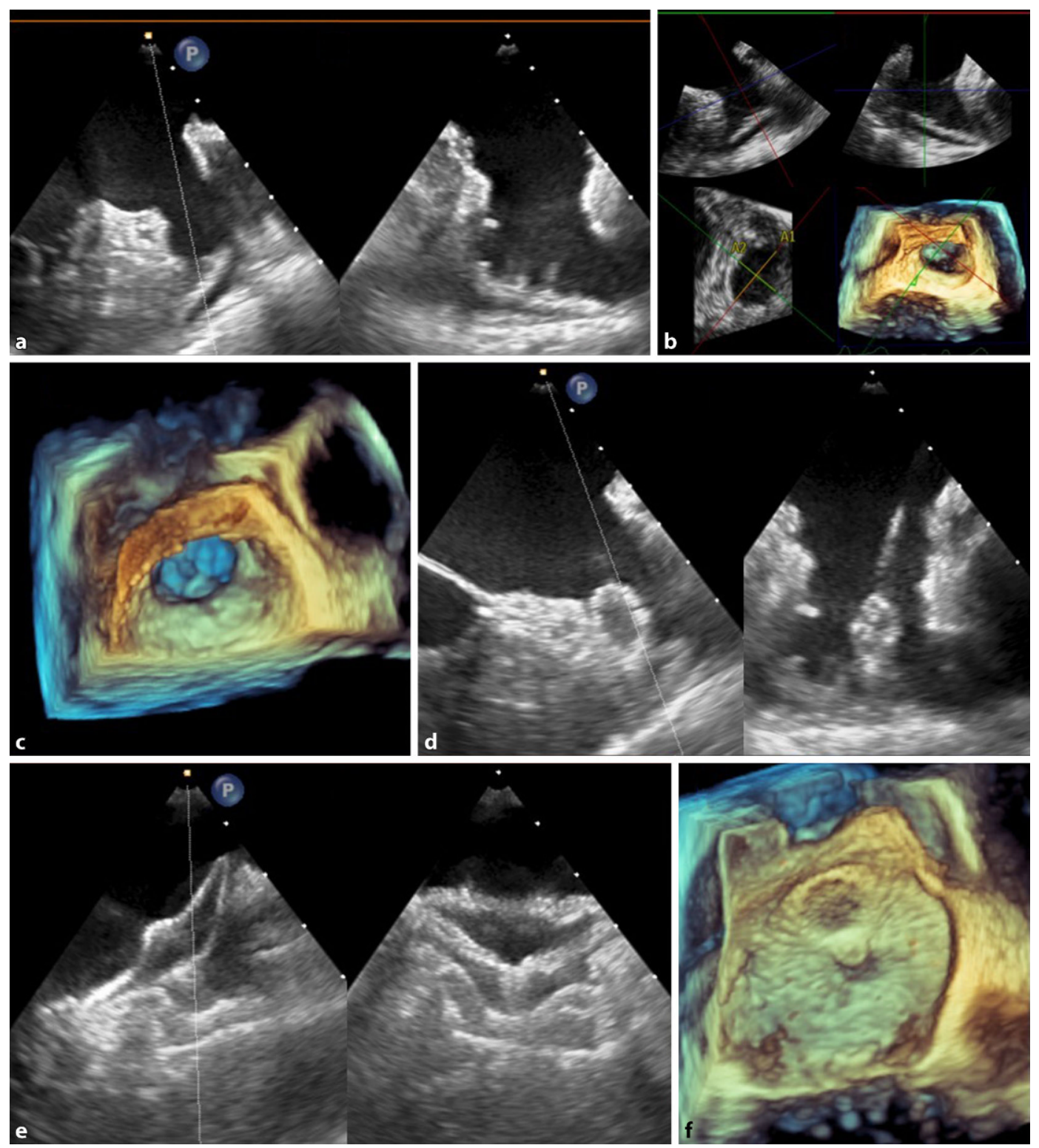

Abb. 94 Evaluation von Morphologie und Größe des linken Vorhofohrs (LAA) in biplaner Darstellung (a), mittels multiplaner Rekonstruktion (b); En-face-Blick auf das LAA-Ostium (c); Sondierung des LAA mit teilentfaltetem Device in biplaner Darstellung (d); Kontrolle der korrekten Device-Position in biplaner (e) und multidimensionaler Darstellung (f)

ne Intubationsnarkose [86, 101, 119]. Das echokardiographische Monitoring umfasst die aktuelle Analyse von Morphologie und Größe des LAA, die transseptale Punktion, die Device-Implantation und die Ergebniskontrolle. Zu Beginn der Intervention muss der Ausschluss einer LAA-Thrombosierung erfolgen. Danach erfolgt die (erneute) Analyse von Morphologie und Größe des LAA. Für die Wahl der adäquaten Device-Größe muss die sog. Landezone des Devices im Bereich des Halses des LAA identifiziert und vermessen werden. Sie liegt distal des LAA-Ostiums, aber vor der Aufteilung des LAA in seine einzelnen Lobi. Unterschiedliche Device-Designs definieren die Landezone unterschiedlich, grund- sätzlich nutzen aber alle endovaskulären Devices den Hals des LAA für die Verankerung des Devices. Um dem häufig ovalen Querschnitt des LAA-Halses Rechnung zu tragen, muss die Landezone in mindestens 2 unterschiedlichen Schnittebenen vermessen werden. Dies sollte in biplaner oder multidimensionaler Technik erfolgen bei Einstellung der Primärebene des LAA nahe der kurzen Achse der Aortenklappe (Einstellung der Gradzahl um 50 ), da in der perpendikulären Ebene normalerweise die größte Ausdehnung des LAA-Halses zu messen ist. Der größte Diameter der Landezone ist dann für die Wahl der Device-Größe entscheidend. Die in dieser Anlotung im 3D-Datensatz mittels MPR vorgegebe- nen Schnittebenen entsprechen denen der biplanen Darstellung. Um eine zu kleine Device-Wahl zu verhindern, muss die Landezone in der Kammersystole bzw. in der Vorhofdiastole bei normaler Vorhoffüllung analysiert werden. Konkrete morphologische Aspekte, die Kontraindikationen für einen Verschluss darstellen, oder besondere LAA-Morphologien, die eine spezielle Implantationstechnik erfordern, müssen zudem detektiert werden. So kann z. B. ein sehr kurzer LAA-Hals bei weit nach ostial reichenden Lobi ein Ausschlusskriterium für eine endovaskuläre Implantation sein und ein proximales Abknicken des Hauptlobus eine spezielle Positionierung des Devices (sog. Sandwich-Technik) erfordern. 
Die räumliche Nähe und die Beschaffenheit der an das LAA angrenzenden Strukturen (die Mitralklappe medial sowie die Perikardumschlagfalte posterior) lassen sich schnell in einem 3D-Volumen der lateralen Vorhofwand erfassen. Hierbei empfiehlt es sich, die Orientierung des 3DVolumens immer gleich zu wählen. Das TEE-Monitoring der transseptalen Punktion ist nicht nur aus Sicherheitsaspekten wichtig, sondern auch im Hinblick auf die Durchführung der Prozedur, da in der Regel nur eine posterior-inferiore Position der Punktion durch die Fossa ovalis eine optimale Ansteuerung des LAA mit der Device-Schleuse ermöglicht. Eine adäquate Punktionsposition besteht, wenn bei posteriorem Tenting in der kurzen Achse das LAA-Ostium dargestellt werden kann. Für das Ansteuern des LAA mit der Device-Schleuse bietet sich ein übersichtlich großes 3D-Volumen des LAA an. Die Sondierung des LAA mit dem nicht oder gering entfalteten Device sollte unter biplaner Anlotung erfolgen. Hierbei gilt es, die Schnittebenen so zu wählen, dass die Aufteilung des LAA in seine Lobi gut zur Darstellung kommt und diese so gezielt intubiert werden können. Die Positionierung und Entfaltung des Devices erfolgt unter biplaner oder multidimensionaler Kontrolle, um die Kompression des Device-Körpers zu evaluieren und nach Verschlusslücken zu suchen (engl. "gaps“). Die multidimensionale Darstellung hilft besonders, die Orientierung und Entfaltung des Devices im LAA in Relation zur Mitralklappe zu erfassen. Wie bei allen linksatrialen Prozeduren müssen nach Rückzug der DeviceSchleuse in das rechte Atrium die Größe des iatrogenen atrialen Septumdefektes und die Richtung des darüber bestehenden Shunts bestimmt werden. Ebenfalls sind während und am Ende der Prozedur Komplikationen, wie z. B. Einrisse des interatrialen Septums oder eine Perikardtamponade, zu detektieren.

Der interventionelle Verschluss des offenen Foramen ovale (PFO)

Das TEE-Monitoring beim Verschluss des offenen Foramen ovale (PFO) bestimmt die Auswahl des Devices und seiner Größe im Hinblick auf die Morphologie des PFO und des interatrialen Septums (• Abb. 10;
[41, 42, 94, 95]). Das Prinzip des interventionellen PFO-Verschlusses basiert darauf, den PFO-Kanal der ausgebliebenen Fusion von Septum primum und secundum über einen Doppelschirm mit mittlerem Steg zu verschließen. Dabei wird ein Teil des Doppelschirms auf der linksatrialen Seite geöffnet und an den Defekt herangezogen, sodass anschließend der verbliebende Teil des Schirms auf der rechtsatrialen Seite geöffnet werden kann, und der Steg zwischen den Schirmchen im Kanal verbleibt. Durch Zug auf beide Schirmchen wird das PFO verschlossen. Prinzipiell muss der linksatriale Schirm größer als der linksatriale Ausgang des PFO-Kanals sein. Ein Ziel der periinterventionellen Analyse ist das Ausmessen der Breite des PFO-Tunnels unmittelbar an seinem Ausgang in den linken Vorhof. Die Dimensionen des PFO-Kanals können biplan oder im 3-D-Datensatz mittels MPR gut dargestellt werden, wobei die primäre Schallebene den PFO-Kanal längs erfassen sollte, damit die Sekundärebene quer zur Tunnelachse steht. Mit einem Sweep der Sekundärebene kann der Tunnel direkt am Ausgang erfasst und ausgemessen werden. Bei kleinem PFO ist es hilfreich, die Messung der PFO-Morphologie nach Sondierung und Offenhalten durch einen Führungsdraht vorzunehmen. Die multidimensionale Darstellung der linksatrialen Öffnung des PFO sollte die Fossa ovalis zur besseren Orientierung immer mit der Aortenklappe zeigen. Die Sondierung des PFO-Tunnels ist in der Regel einfach, jedoch kann bei kleinem PFO oder atypischer PFO-Position ein echokardiographisches "Guiding" helfen. Die Dokumentation der Entfaltung des linksatrialen Schirms und sein Heranführen an das Septum erfolgen biplan oder im 3D-Modus unter Längsdarstellung des Katheters in der Primärebene. In dieser Anlotung erfolgen auch die Entfaltung des rechtsatrialen Schirms und das Freisetzen des Devices. Vor dem Freisetzen des Devices muss sicher festgestellt werden, ob das interatriale Septum von beiden Seiten komprimiert wird und das Device adäquat der Aortenwurzel anliegt.
Der interventionelle Verschluss des Vorhofseptumdefekts (ASD)

Bei interventionellen Verschlüssen von Vorhofseptumdefekten (ASD) ist die genaue präinterventionelle Analyse mittels TEE eine wesentliche Voraussetzung für eine erfolgreiche Intervention (• Abb. 11; [95]). Für einen interventionellen ASD-Verschluss eignet sich v. a. der in der Fossa ovalis gelegene ASD vom Secundum-Typ. Der weiter superior oder inferior im Septum liegende ASD vom Sinus-venosus-Typ ist häufig mit fehlmündenden Pulmonalvenen assoziiert [6]. Sein interventioneller Verschluss ist allerdings die Ausnahme und kann nur mit speziellen Devices erfolgen. Multiple ASDs, das fenestrierte interatriale Septum oder sehr große ASDs vom SecundumTyp mit fehlenden Randzonen können ebenfalls Kontraindikationen für ein interventionelles Vorgehen darstellen. Die exakte Vermessung der ASD-Größe kann mittels multipler 2D-TEE-Sequenzen oder in einem 3D-TEE-Datensatz erfolgen.

Neben dem maximalen Durchmesser des ASD ist das Ausmaß des verbliebenen Randes des interatrialen Septums, dem sog. „rim“, wichtig, um den ASD zu analysieren. Die ASD-Größe kann zudem mit einem maximal entfalteten Sizing-Ballon, der sog. "Stop-flow"-Methode, durch biplane Darstellung oder besser in einem 3D-Datensatz durch MPR (maximaler und minimaler Defektdiameter) bestimmt werden. Der Vorteil der Ballon-Sizing-Methode kann bei mobilem oder flexiblem (engl. "floppy") Septum darin liegen, die funktionelle Defektgröße zu ermitteln. Für die Analyse der ASD-Morphologie bieten sich in der 2D-TEE 3 Schnittebenen an: der 4-Kammer-Blick (Rand des ASD zur Mitralklappe hin), die kurze Achse der Aortenklappe (anteroposteriorer Rand des ASD) und die bicavale Schnittebene (inferosuperiorer Rand des ASD). Die 3D-Technik ermöglicht gegenüber der 2D-Echokardiographie eine einfachere Darstellung des prozentualen Anteils des fehlenden ASD-Randes in der Zirkumferenz sowie eine einfachere Ausmessung des Defektes. In der transversalen ASDSchnittebene können zentral im Defekt die sagittale Schnitteben rotiert werden, um die Randbreite des ASD in jeder Dimension zu ermitteln. Für einen interventionellen 

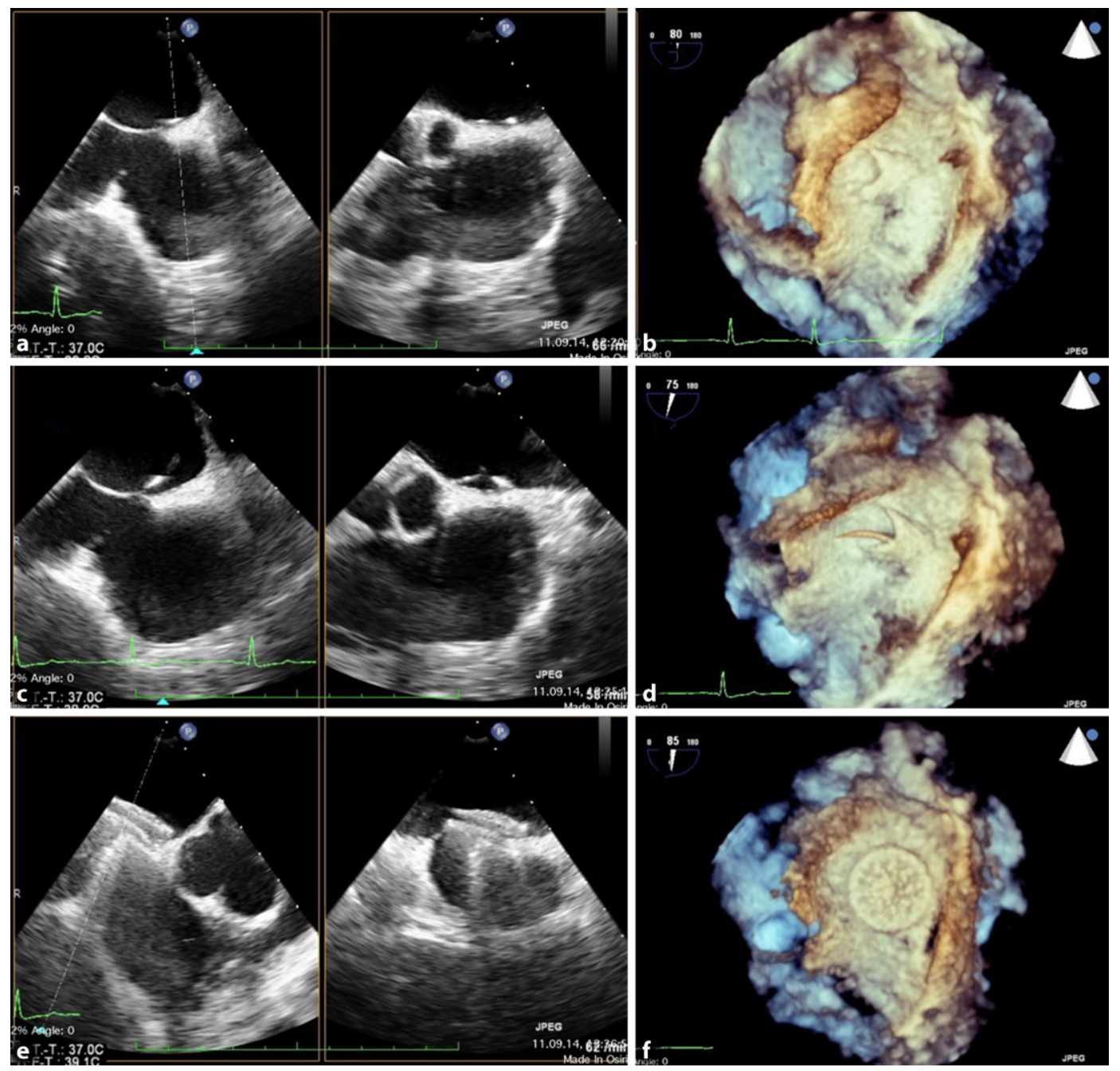

Abb. $10<$ Biplane und multidimensionale Darstellung eines offenen Foramen ovale (PFO) vor $(\mathbf{a}, \mathbf{b})$ und nach (c, d) Drahtsondierung mit Ausrichtung der primären Schnittebene längs zum PFO-Tunnel und der sekundären perpendikulären Schnittebene. Enface-Blick auf die Tunnelbreite am Ausgang in linken Vorhof (c). Überprüfung der Device-Position vor Ablösung in biplaner (e) und multiplaner (f) Darstellung

ASD-Verschluss kann ein fehlender Rand bis zu 30\% der Zirkumferenz - meist anterior und/oder superior - toleriert werden. Eine Kontraindikation für einen DeviceVerschluss ist auch das Fehlen von gegenüberliegenden Defekträndern - meist anterior und posterior -, da ein direkt zwischen Aortenwurzel und Vorhofhinterwand positioniertes Device bei fehlendem "rim" ein hohes Risiko für Gewebeerosionen hat. Beim interventionellen ASDVerschluss ist der wirksame Verschlussteil des Devices der Verbindungssteg zwischen den Schirmen. Daher wird der Durchmesser der üblichen Nitinol-Devices bei zirkulären Defekten ca. $2 \mathrm{~mm}$ größer als der maximal bestimmte Defektdiameter gewählt. Bei stark ovalen ASDs kann eher ein kleineres Device gewählt werden, um einen Einriss des Restseptums zu vermeiden. Bei Defekten mit fehlendem Rand erfolgt häufig ein Oversizing, um das Device sicher zu verankern. Die Dokumentation der Device-Implantation erfolgt in biplaner und/oder 3D-Technik unter Längsdarstellung des Device-führenden Katheters in der Primärebene. So können Device-Schleuse und die Entfaltung beiden Schirmchen sowie ihre Interaktion mit dem angrenzenden „rim" dargestellt werden. Besonders wichtig nach der Implantation ist die Konfiguration des Devices zur Aortenwurzel hin. Die angrenzenden Schirme dürfen wegen möglicher Erosionen nicht gegen die Aortenwand drücken, sondern sollen der Aortenwand anliegen. Mittels Farbdopplerechokardiographie müssen residuelle Shunts detektiert werden. Turbulenzen durch das Device sind direkt nach der Implantation normal, da zum kompletten Verschluss eine Endothelialisierung notwendig ist. Größere Jetformationen am Rand des Verbindungsstegs können sowohl durch eine zu kleine
Device-Größe bei inkomplettem ASD-Verschluss als auch durch eine zu große Device-Größe durch inkomplette Entfaltung bei Kompression durch die verbliebenen Randbereiche bedingt sein. Gegebenenfalls müssen bei solchen Befunden Repositionierungen oder Device-Wechsel vorgenommen werden.

Der interventionelle Verschluss von ventrikulären Septumdefekten und paraprothetischen Leckagen

Das TEE-Monitoring von ventrikulären Septumdefekten (VSDs) und interventionellen Leckagen an Klappenprothesen ist individuell sehr unterschiedlich. Vor der Intervention sind Lokalisation, Größe und hämodynamische Relevanz der Defekte bzw. Leckagen zu analysieren. Eine wesentliche Hilfestellung zur Ausmessung der Defekte und Leckagen bietet die 3D-TEE durch 

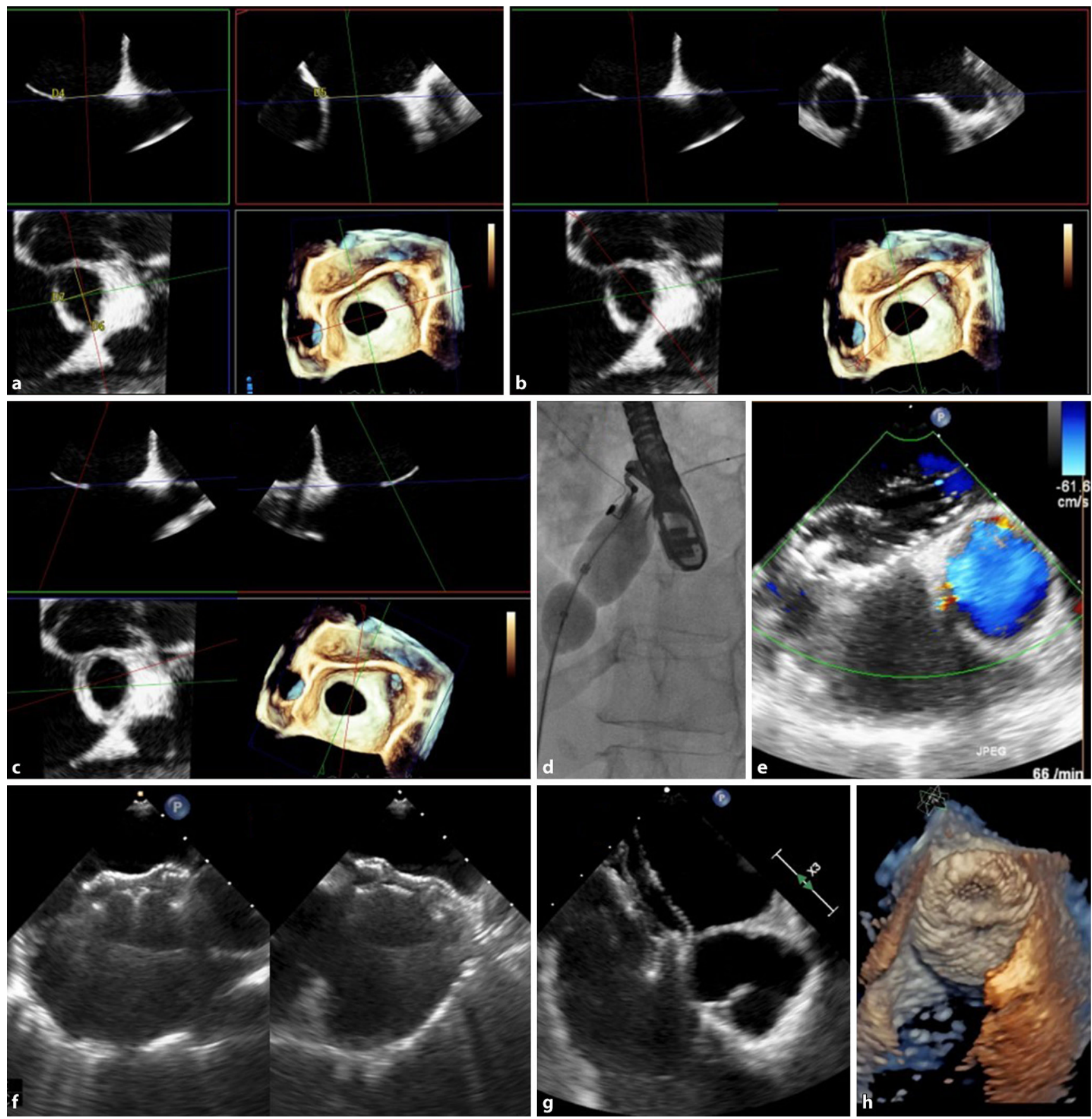

Abb. $11 \Delta$ Multiplanare Rekonstruktion eines zentralen Vorhofseptumdefektes (ASD) vom Secundumtyp mit tranversaler Schnittebene (blau) parallel zur Defektebene (a-c); Ausmessen von minimalem und maximalen ASD-Diameter (a). Analyse der Ausdehnung des anterosuperior fehlenden Defektrandes (b, $\mathbf{c}$ ) in der Orientierung der multidimensionalen En-faceASD-Darstellung mit der Aortenklappe bei "9 Uhr". Die rot markierten Ebenen zeigen den fehlenden „rim" anterior (b) und superior (c) - es fehlt ein Defektrand in ca. $30 \%$ der Zirkumferenz. "Stop-Flow"-Methode mit Ballonokklusion des ASD in der Fluoroskopie (d) und in der 2-D-TEE (e); biplane Kontrolle der korrekten Device-Position (f). Beispiel einer nicht zu akzeptierenden Position des linksatrialen Schirms anterior mit Kompression der Aortenwand (g); multidimensionale En-face-Darstellung eines großen LA-Schirmchens in korrekter Position (h) 
die Akquisition farbkodierter Zoom-Datensätze, da durch die MPR die Regurgitationsöffnungen in der Regel gut vermessen und objektiviert werden können, sofern keine relevanten Artefakte von prothesenbedingten Schlagschatten sich auf diese Regionen projizieren [63]. Auch die Enface-3D-Darstellungen auf die jeweiligen Leckagen können als Basis für die Wahl der geeigneten Okkluder bzw. Schirmchen bei VSD und dessen Größe herangezogen werden [63]. Während der Intervention sind die Präsentation und Dokumentation der Drahtsondierung des Defektes wichtig. Das Vorschieben der jeweiligen Okkluder in die ventrikulären und paravalvulären Leckagen sollte genau dokumentiert werden. Die adäquate Entfaltung der implantierten Okkluder kann fluoroskopisch und echokardiographisch kontrolliert werden. Echokardiographisch müssen potenzielle Interaktionen der Okkluder mit angrenzenden Strukturen ausgeschlossen werden. Falls nach Leckagenverschluss Turbulenzen mit hohen Flussgeschwindigkeiten fortbestehen, müssen alternative Therapieoptionen diskutiert werden, da in diesen Fällen ein hohes Risiko für eine postinterventionelle Hämolyse besteht.

\section{Die Echokardiographie bei tempo- rären und permanenten kardialen Unterstützungssystemen}

In Ergänzung zum Monitoring von Interventionen sind die TTE und TEE auch bei der Implantation und Kontrolle von temporären Herzunterstützungssystemen notwendig. Hierbei ist die genaue Lagekontrolle bei der Platzierung der Ansaugkanülen zu beachten, da eine zu geringe Distanz zur Vorhof- bzw. Ventrikelwand zum Ansaugen der Herzwände an die Kanüle mit der Folge der iatrogenen Perforation führen kann. Auch bei retrograd arteriell über die Aortenklappe implantierten Pumpsystemen müssen Ansaug- und Auslassbereich der Kanülen eindeutig dokumentiert werden, um Fehlfunktionen des Unterstützungssystems zu vermeiden (• Abb. 12).

Ebenfalls spielt die Echokardiographie bei der postoperativen Versorgung von Patienten mit permanenten linksventrikulären kardialen Unterstützungssystemen (LVAD $=$ "left ventricular assist devices") eine zunehmend wichtige Rolle. LVADs sind
Zentrifugalpumpen, die apikal implantiert werden. Das Device saugt das Blut durch die Einflusskanüle aus dem linken Ventrikel an und pumpt es über den sog. "Outflow Graft" in die Aorta (- Abb. 12). Von der Pumpe wird ein Kabel, die "Driveline", subkutan getunnelt aus dem Körper ausgeleitet. Sie verbindet das Device mit den Batterien und einer Steuereinheit, dem sog. „Controller". An der Steuereinheit kann u. a. der grob kalkulierte Blutfluss durch das Gerät abgelesen werden. Mit den aktuell üblicherweise implantierten Systemen, lassen sich gute Ergebnisse bezüglich des Langzeitüberlebens und der Lebensqualität erzielen $[15,22,23,48,70,97,98,103$, $109,110]$. Da der linke Ventrikel durch das Device unterstützt wird, ist seine Funktion primär nicht erforderlich. Er kann aber an der Erzeugung des Herzzeitvolumens teilnehmen. Wenn dies der Fall ist, öffnet sich die Aortenklappe. Die Blutdruckkurve ist dann pulsatil. Ansonsten besteht durchgehend ein Mitteldruck.

Der echokardiographische Untersuchungsgang bei LVADs sollte standardisiert erfolgen und spezielle Fragestellungen berücksichtigen. Bei jeglichen Auffälligkeiten bestehen zusätzlich spezifische Fragestellungen, sodass die Patienten dann speziellen Nachsorgezentren zuzuführen sind [15, $31,48,98]$. Dem echokardiographischen Befund sollten immer auch der aktuelle Blutdruck des Patienten sowie der LVADFluss bzw. die LVAD-Einstellung beigefügt werden. Die Bildqualität des parasternalen Schallfensters ist bei LVAD-Patienten normalerweise gut. Da das Device apikal lokalisiert ist, lassen sich linker und rechter Ventrikel von apikal in der Regel nur verkürzt abbilden. Die Dopplerspektren der regionalen Blutflussgeschwindigkeiten von Inflow-Kanüle und Outflow-Grafts sind oft Artefakt-überlagert. Daher sind Dopplerflussmessungen selten durchführbar. Da eine Bestimmung der LV-Funktion mittels Planimetrie meist nicht möglich ist, empfiehlt sich die Bestimmung der LV "fractional area change" (FAC) zur globalen Beurteilung der LV-Funktion. Parasternale und nicht standardisierte Darstellungen des RV-Einflusstraktes sind sinnvoll, da die apikale Darstellung der Trikuspidalklappe häufig nicht optimal gelingt. Die TAPSE ist nach LVAD-Implantation wie auch nach anderen herzchirurgischen Eingrif- fen reduziert [92]. Zur Beurteilung der RV-Funktion ist deshalb die rechtsventrikuläre FAC sinnvoller. Die RV-Funktion und speziell RV-Vorlast sind durch die Messung des V.-cava-inferior-Diameters und der Atemvariabilität des Blutflusses in den systemischen Venen zu charakterisieren. Eine Einschränkung der RV-Funktion und/oder eine hochgradige Trikuspidalklappeninsuffizienz können zur Reduktion der Lungenperfusion und konsekutiv zur Reduktion des LV-Einstroms führen. Dadurch wird der linke Ventrikel kleiner bei gleichzeitigem Abfall des Herzzeitvolumens durch zu geringen Fluss des LVAD. Typische Zeichen der Rechtsherzinsuffizienz sind zudem dilatierte Hohlvenen, Pleuraergüsse, Aszites und Ödeme. Die Aortenklappe ist bei Patienten am LVAD von besonderer Bedeutung. Bei durchgehend geschlossener Klappe besteht eine kontinuierliche Klappenbelastung, die die Entstehung einer Al begünstigt [110]. Die Quantifizierung der Al bei Patienten am LVAD kann schwierig sein [31], wenn insbesondere bei durchgehend geschlossener Klappe eine systolische und diastolische Regurgitation vorliegt. Eine relevante Al führt zu einem „Kurzschluss" des Blutflusses zwischen Device und Ventrikel. Diese Konstellation ist prognostisch ungünstig $[22,23,93]$. Deswegen sollte die LVADEinstellung so justiert werden, dass sich die Aortenklappe intermittierend öffnet [22, 23]. Eine derartige Einstellung kann auch der Gefahr von Thrombenbildungen im Aortenbulbus vorbeugen. Eine MI ist nach LVAD-Implantation häufig deutlich rückläufig. Auch eine schwere $\mathrm{Ml}$ wird meistens hämodynamisch gut toleriert. Ein plötzlicher MI-Progress nach LVADImplantation dagegen kann für eine nicht ausreichende LV-Drainage bzw. „Entlastung" durch das Device sprechen.

Besondere Szenarien stellen die Pumpenthrombose und die signifikante linksventrikuläre Unterfüllung dar. Sollte sich eine Thrombose im LVAD selbst oder im Bereich des LVAD-Einflusses befinden, so steigt in der Regel die "Wattzahl“ des Gerätes am Controller. Typisch in dieser Situation sind zudem eine Hämolyse mit Anstieg der Lactatdehydrogenase und des Bilirubins sowie ein Abfall des Haptoglobins [97]. Echokardiographisch führt der Abfall der effektiven Pumpleistung des LVAD 

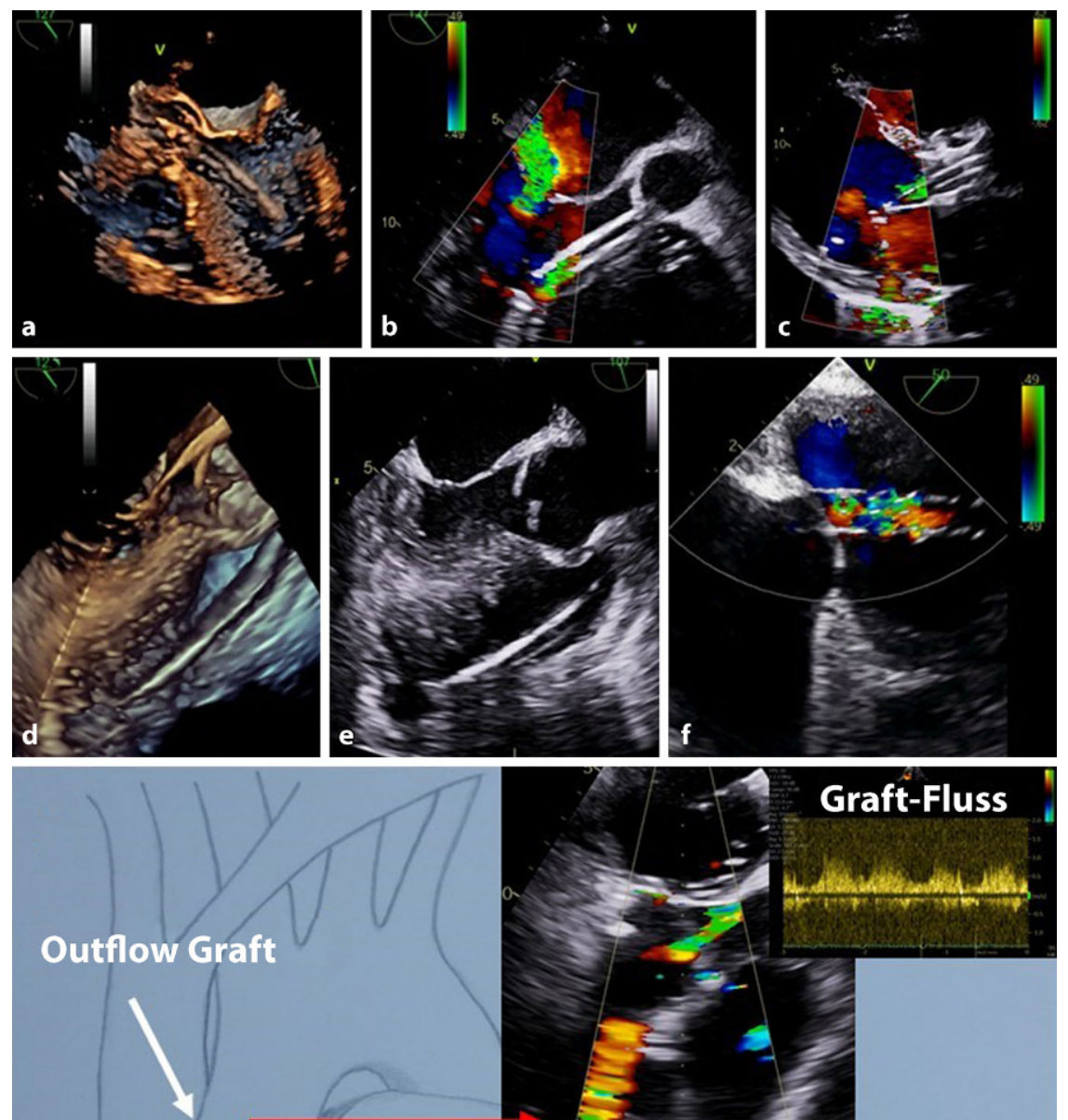

Graft-Fluss
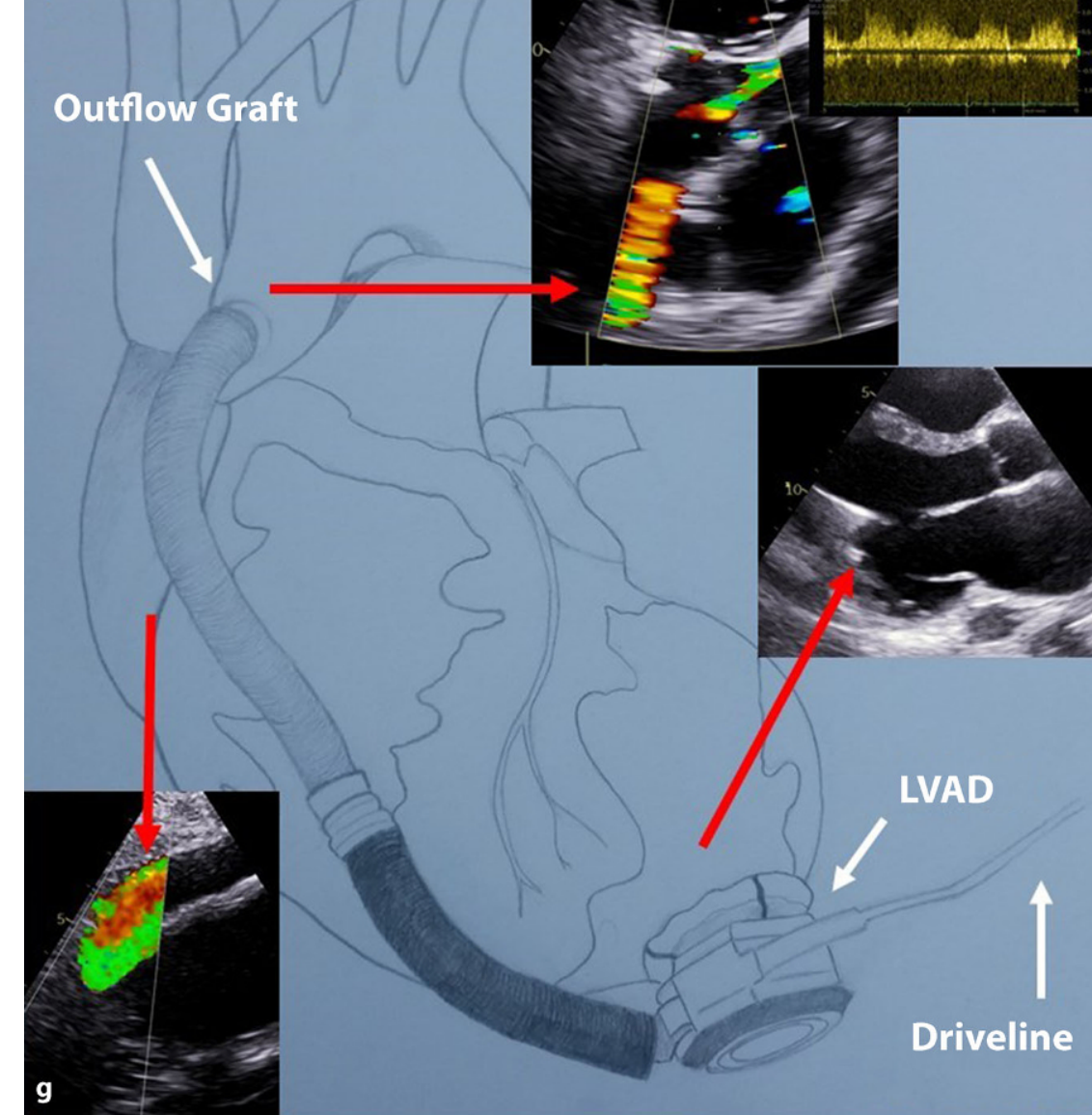

Abb. 12 \ Darstellung von temporären kardialen Unterstützungssystemen mittels 3D-TEE (a) sowie farbkodierter 2D-TEE (b) bei regelrechter Position. Farbkodierte Darstellung einer Fehlposition des Ansaugbereiches an der posterioren LV-Wand (c) in der farbkodierten 2D-TTE. Darstellung eines temporären Rechtsherzunterstützungssystems in der 3D-TEE (d) sowie 2D-TEE (e). Darstellung eines temporären Linksherzunterstützungssystems in der farbkodierten 2D-TEE (f). Schematische Darstellung eines permanenten Linksherzersatzsystems (LVAD) mitDarstellung des Inflow-Bereiches (LVADmitDriveline) sowie des sog. "Outflow Graft" (Ausflussbereich in die Aorta ascendens. Zusätzlich sind der Inflow-Bereich, der Graft-Fluss und der Outflow-Bereich in repräsentativen echokardiographischen Dokumentationen eingefügt. [Schemazeichnung der Teilabbildung g: mit freundl. Genehmigung von $\odot$ Louise Hagendorff (2021)]. All Rights reserved) zu einer LV-Dilatation, einer Öffnung der Aortenklappe bei jedem Herzschlag, einem MI-Progress und einem sPAP-Anstieg bzw. einer Einschränkung der RV-Funktion bis zur Rechtsherzdekompensation. Im Rahmen einer Sepsis mit Abfall des systemischen Widerstandes oder bei einer Rechtsherzinsuffizienz kann es zu einer reduzierten Füllung des linken Ventrikels kommen, wodurch der Fluss des LVADs abnimmt. In dieser Situation verkleinert sich der linke Ventrikel bei anhaltend geschlossener Aortenklappe. Das interventrikuläre Septum verlagert sich signifikant nach links, was zu einer passiven Dilatation des Trikuspidalklappenrings mit RV-Dilatation sowie Reduktion der RV-Funktion bis zur RV-Dekompensation führt. In Extremfällen ist die Mitralklappe durchgehend offen.

\section{Vorsichtsmaßnahmen bei der Echokardiographie hochinfektiöser Patienten in Pandemien}

Bei hochinfektiösen Patienten - insbesondere auch im Hinblick auf die immer noch aktuelle COVID-19-Pandemie - sind häufig echokardiographische Untersuchungen indiziert, da parallel zum Infektionsgeschehen kardiovaskuläre Erkrankungen bei diesen Patienten vorkommen, jedoch auch kardiale Komplikationen durch die Infektion induziert werden können. Zu diesen kausalen Folgen gehören Störungen des Gerinnungssystems u.a. mit den Folgen eines Myokardinfarkts oder einer Lungenembolie, aber auch die Entwicklung von Myokarditiden, akuter Herzinsuffizienz und Arrhythmien [107]. Somit sollten diese Patienten bei Auftreten von Symptomen, die auf eine kardiovaskuläre Erkrankung hinweisen, bei EKG-Veränderungen und bei ansteigenden kardialen Biomarkern echokardiographiert werden. Damit ist bei dieser Patientengruppe die Echokardiographie indiziert, sofern eine therapeutische Konsequenz von der 
echokardiographischen Untersuchung abhängt.

Wegen des Risikos der Keimübertragung innerhalb der jeweiligen Institutionen sowie der Ansteckungsgefahr für das Personal müssen besondere Vorsichtsmaßnahmen und Hygienevorschriften unter diesen Bedingungen eingehalten werden, die in der aktuellen COVID-19Pandemie eine aktuelle Relevanz hatten. Durch die Impfung des Personals ist derzeit die Gefahr minimiert, ist jedoch bei neuen Virusmutationen bzw. Virusarten zu bedenken. Neben der eindeutigen Indikationsstellung zur Echokardiographie sollten bei hochinfektiösen Patienten je nach Gefährdung die Untersucher Schutzkleidung (insbesondere Handschuhe), Schutzmasken und Schutzbrillen tragen. Ärzte, die ein spezielles Gesundheitsrisiko haben, sollten von TTE- und TEE-Untersuchungen ausgeschlossen werden. Bei nachgewiesener Infektion der Untersucher im Rahmen der Patientenbehandlung sind diese von weiteren Untersuchungen zu isolieren. Jegliche TTE-Untersuchung sollte bei hoher Infektionsgefahr verkürzt und fokussiert durchgeführt werden, um die Expositionszeiten zwischen Untersucher und Patient zu minimieren. Eine besondere Situation besteht bei TEE-Untersuchungen, da das medizinische Personal durch Rachenbetäubung, Husten der Patienten beim Einführen der Sonde sowie durch Kontamination der endoskopischen Geräte mit Aerosolen und kontaminierten Flüssigkeitspartikeln in Kontakt kommen kann, was einem stark erhöhten Ansteckungsrisiko für den Arzt*innen bedeutet. Daher sollte bei derartigen Patienten eine TEE nur durchgeführt werden, wenn eine direkte Therapieentscheidung davon abhängt. Elektive TTE- und TEEUntersuchungen sind zu verschieben. Die Echokardiographie sollte mit möglichst geringem Zubehör (ggf. auch unter Verzicht des 1-Kanal-EKGs) durchgeführt werden, da sich Untersucher sowie UItraschalleinheit nach jeder Untersuchung einer Oberflächendesinfektion (Schallköpfe mit den üblichen Detergenzien; Oberfläche, Leitungen und Knöpfe des Ultraschallgerätes) unterziehen müssen. TEE-Sonden sind inklusive Griff, Kabel und Stecker im Patientenraum zu desinfizieren. Bei einer TEE sollten maximal 2 Personen neben dem Patienten im Raum sein, Türen und Zwischentüren sind geschlossen zu halten. Nach TTE- und TEE-Untersuchung sollte bei hoher Infektionsgefahr (insbesondere durch Viren) ausgiebig und gründlich gelüftet werden. Weiterhin sind Ultraschallgeräte, die ausschließlich auf Infektionsstationen zum Einsatz kommen, zu befürworten, da Transporte von infizierten Patienten vermieden werden sollten. Der Einsatz von Kleingeräten ist im Hinblick auf die notwendigen einzusetzenden Ultraschallmodalitäten abzuwägen. Bildbeurteilungen sollten nach der Untersuchung an einer anderen Lokalisation an getrennten Rechnern außerhalb des Infektionsbereiches durchgeführt werden.

\section{Fazit für die Praxis}

- Das vorliegende Manual über erweiterte echokardiographische Modalitäten sowie den Einsatz der Echokardiographie bei speziellen Indikationen kann nur einen Überblick über die diagnostischen Möglichkeiten der Echokardiographie geben.

- Es ist offensichtlich, dass aufgrund der erheblichen Diversifikation der Methode für die jeweilig auftretenden Fragestellungen bei oft sehr speziellen und auch seltenen Patientengruppen intensive Kenntnisse notwendig sind.

- Die Untersucherexpertise bezieht sich dabei sowohl auf die Gerätekenntnis zur Bildoptimierung und zur Durchführung bestimmter Methoden als auch auf das notwendige anatomische, pathologische, physiologische und pathophysiologische Wissen zu den jeweiligen Krankheitsentitäten.

- Damit kann diese Expertise nicht in kurzer Zeit erlernt werden. Die Anwendung des gesamten Armamentariums der erweiterten Echokardiographie bedeutet eine lange Ausbildungszeit.

- Wie in allen Bereichen der Kardiologie und Medizin können die diversen Möglichkeiten der erweiterten Echokardiographie nur durch fundiertes Spezialwissen hinreichend ausgeschöpft werden.

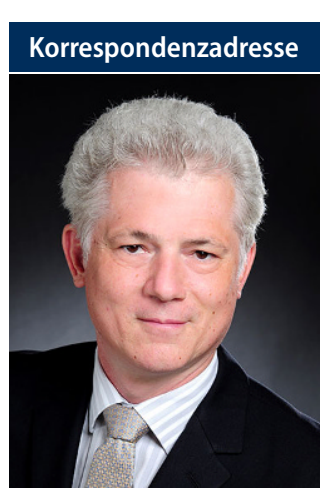

Prof. Dr. Andreas Hagendorff

Klinik und Poliklinik für Kardiologie, Universitätsklinikum Leipzig

Liebigstr. 20, 04103 Leipzig, Deutschland

andreas.hagendorff@medizin.uni-leipzig.de

Danksagung. Die Schemazeichnung der

- Abb. 12 wurde von Louise Hagendorff angefertigt. Louise Hagendorff hat die Veröffentlichung der Zeichnung genehmigt.

\section{Einhaltung ethischer Richtlinien}

Interessenkonflikt. Den Interessenkonflikt der Autoren finden Sie online auf der DGK-Homepage unter http://leitlinien.dgk.org/ bei der entsprechenden Publikation.

Für diesen Beitrag wurden von den Autoren keine Studien an Menschen oder Tieren durchgeführt. Für die aufgeführten Studien gelten die jeweils dort angegebenen ethischen Richtlinien.

\section{Literatur}

1. Alessandrini $H$, Kreidel F, Schlüter $M$, Frerker $C$, Schmidt T, Thielsen T, Schäfer U, Kuck KH (2017) Prognostic implication of post-MitraClip vena contracta area in heart failure patients with functional mitral regurgitation. Eurolntervention 12:1946-1953. https://doi.org/10.4244/EIJ-D-1600190

2. Badano LP, Kolias TJ, Muraru D, Abraham TP, Aurigemma G, Edvardsen T, D'Hooge J, Donal E, Fraser AG, Marwick T, Mertens L, Popescu BA, Sengupta PP, Lancellotti P, Thomas JD, Voigt JU, Industry representatives (2018) Standardization of left atrial, right ventricular, and right atrial deformation imaging using two-dimensional speckle tracking echocardiography: a consensus document of the EACVI/ASE/Industry Task Force to standardize deformation imaging. Eur Heart J Cardiovasc Imaging 19:591-600. https://doi.org/ 10.1093/ehjci/jey042

3. Badano LP, Addetia K, Pontone G, Torlasco C, Lang RM, Parati G, Muraru D (2020) Advanced imaging of right ventricular anatomy and function. Heart 106:1469-1476. https://doi.org/10.1136/ heartjnl-2019-315178

4. Baldus S, Kuck KH, Rudolph V, NefH, Eggebrecht H, Boekstegers $P$, Wöhrle J, Ince $H$, Möllmann $H$, Stellbrink C, Hausleiter J, von Bardeleben S, Kelm M, Elsässer A (2018) Interventionelle The- 
rapie von AV-Klappenerkrankungen - Fokus Mitralklappeninsuffizienz. Positionspapier der Deutschen Gesellschaft für Kardiologie. Kardiologe 12:128-144. https://doi.org/10.1007/s12181018-0232-y

5. Baumgartner H, Falk V, Bax JJ, De Bonis M, Hamm C, Holm PJ, lung B, Lancellotti $P$, Lansac E, Rodriguez Muñoz D, Rosenhek R, Sjögren J, Tornos Mas $P$, Vahanian A, Walther T, Wendler $\mathrm{O}$, Windecker $\mathrm{S}$, Zamorano JL, ESC Scientific Document Group (2017) 2017 ESC/EACTS Guidelines for the management of valvular heart disease. Eur Heart J 38:2739-2791. https://doi.org/10.1093/ eurheartj/ehx391

6. Baumgartner $\mathrm{H}$, De Backer J, Babu-Narayan SV Budts W, Chessa M, Diller GP, Lung B, Kluin J, Lang IM, Meijboom F, Moons P, Mulder BJM, Oechslin E, Roos-Hesselink JW, Schwerzmann M, Sondergaard L, Zeppenfeld K, ESC Scientific Document Group (2021) 2020 ESC Guidelines for the management of adult congenital heart disease. Eur Heart J 42:563-645. https://doi.org/ 10.1093/eurheartj/ehaa554

7. Biaggi P, Felix C, Gruner C, Herzog BA, Hohlfeld S, Gaemperli O, Stähli BE, Paul M, Held L, Tanner FC, Grünenfelder J,Corti R, BettexD (2013) Assessment of mitral valve area during percutaneous mitral valve repair using the MitraClip system: comparison of different echocardiographic methods. Circ Cardiovasc Imaging 6:1032-1040. https://doi.org/ 10.1161/CIRCIMAGING.113.000620

8. Bleakley C, Monaghan M (2020) 3D transesophageal echocardiography in TAVR. Echocardiography 37:1654-1664. https://doi.org/10.1111/echo. 14777

9. Brochet $E$, Détaint $D$, Fondard O, Tazi-Mezalek A, Messika-Zeitoun D, lung B, Vahanian A (2011) Early hemodynamic changes versus peak values: what is more useful to predict occurrence of dyspnea during stress echocardiography in patients with asymptomatic mitral stenosis? J Am Soc Echocardiogr 24:392-398. https://doi.org/10. 1016/j.echo.2011.01.006

10. Brogi E, Gargani L, Bignami E, Barbariol F, Marra A, Forfori F, Vetrugno L (2017) Thoracic ultrasound for pleural effusion in the intensive care unit: a narrative review from diagnosis to treatment. Crit Care 21:325. https://doi.org/10.1186/s13054017-1897-5

11. Buck T, Breithardt $O A$, Faber $L$, Fehske W, Flachskampf FA, Franke A, Hagendorff A, Hoffmann R, Kruck I, Kucherer H, Menzel T, Pethig K, Tiemann K, Voigt JU, Weidemann F, Nixdorff U (2009) Manual zur Indikation und Durchfuhrung der Echokardiografannuie. Clin Res Cardiol Suppl 4:3-51. https:// doi.org/10.1007/s11789-009-0051-6

12. Carabello BA (2005) Modern management of mitral stenosis. Circulation 112:432-437. https:// doi.org/10.1161/CIRCULATIONAHA.104.532498

13. Clavel MA, Burwash IG, Mundigler G, Dumesnil JG, Baumgartner $H$, Bergler-Klein J, Sénéchal $M$, Mathieu P, Couture C, Beanlands R, Pibarot P (2010) Validation of conventional and simplified methods to calculate projected valve area at normal flow rate in patients with low flow, low gradient aortic stenosis: the multicenter TOPAS (True or Pseudo Severe Aortic Stenosis) study. J Am Soc Echocardiogr 23:380-386. https://doi.org/10. 1016/j.echo.2010.02.002

14. Clavel MA, Ennezat PV, Maréchaux S, Dumesnil JG, Capoulade R, Hachicha Z, Mathieu P, Bellouin A, Bergeron $S$, Meimoun P, ArsenaultM, LeTourneauT, Pasquet A, Couture C, Pibarot P (2013) Stress echocardiography to assess stenosis severity and predict outcome in patients with paradoxical lowflow, low-gradient aortic stenosis and preserved LVEF. JACC Cardiovasc Imaging 6:175-183. https:// doi.org/10.1016/j.jcmg.2012.10.015

15. Cohen DG, Thomas JD, Freed BH, Rich JD, Sauer AJ (2015) Echocardiography and continuous-flow left ventricular assist devices: evidence and limitations. JACC Heart Fail 3:554-564. https://doi. org/10.1016/j.jchf.2015.03.003

16. Corrigan FE, Gleason PT, Condado JF, Lisko JC, Chen JH, Kamioka N, Keegan P, Howell S, Clements SD Jr, Babaliaros VC, Lerakis S (2019) Imaging for predicting, detecting, and managing complications after transcatheter aortic valve replacement. JACC Cardiovasc Imaging 12:904-920. https://doi.org/10.1016/j.jcmg.2018. 07.036

17. Corrigan FE, Hall MJ, Iturbe JM, Condado JF, Kamioka N, Howell S, Thourani VH, Clements SD, Babaliaros VC, Lerakis S (2019) Radioprotective strategies for interventional echocardiographers during structural heart interventions. Catheter Cardiovasc Interv 93:356-361. https://doi.org/10. $1002 /$ ccd. 27843

18. Donal E, Mascle $S$, Brunet A, Thebault C, Corbineau $H$, Laurent $M$, Leguerrier A, Mabo P (2012) Prediction of left ventricular ejection fraction 6 months after surgical correction of organic mitral regurgitation: the value of exercise echocardiography and deformation imaging. Eur Heart J Cardiovasc Imaging 13:922-930. https://doi.org/ 10.1093/ehjci/jes068

19. Donal E, Lip GY, Galderisi M, Goette A, Shah D, Marwan M, Lederlin M, Mondillo S, Edvardsen T, Sitges M, Grapsa J, Garbi M, Senior R, Gimelli A, Potpara TS, Van Gelder IC, Gorenek B, Mabo P, Lancellotti P, Kuck KH, Popescu BA, Hindricks G, Habib G, Cardim NM, Cosyns B, Delgado V, Haugaa KH, Muraru D, Nieman K, Boriani G, Cohen A (2016) EACVI/EHRA Expert Consensus Document on the role of multi-modality imaging for the evaluation of patients with atrial fibrillation. Eur Heart J Cardiovasc Imaging 17:355-383. https://doi.org/10.1093/ehjci/jev354

20. Deferm S, Bertrand PB, Churchill TW, Sharma R, Vandervoort PM, Schwamm LH, Yoerger Sanborn DM (2021) Left atrial mechanics assessed early during hospitalization for cryptogenic stroke are associated with occult atrial fibrillation: a speckle-tracking strain Echocardiography study. J Am Soc Echocardiogr 34:156-165. https://doi. org/10.1016/j.echo.2020.09.009

21. Di Salvo G, Miller O, Babu Narayan S, Li W Budts W, Valsangiacomo Buechel ER, Frigiola A, van den Bosch $A E$, Bonello $B$, Mertens $L$, Hussain T, Parish V, Habib G, Edvardsen T, Geva T, Baumgartner H, Gatzoulis MA, 2016-2018 EACVI Scientific Documents Committee (2018) Imaging the adult with congenital heart disease: a multimodality imaging approach-position paper from the EACVI. Eur Heart J Cardiovasc Imaging 19:1077-1098. https://doi.org/10.1093/ehjci/ jey 102

22. Estep JD, Starling RC, Horstmanshof DA, Milano $C A$, Selzman $C H$, Shah KB, Loebe $M$, Moazami N, Long JW, Stehlik J, Kasirajan V, Haas DC, O'Connell JB, Boyle AJ, Farrar DJ, Rogers JG, ROADMAP Study Investigators (2015) Risk assessment and comparative effectiveness of left ventricular assist device and medical management in ambulatory heart failure patients: results from the ROADMAP study. J Am Coll Cardiol 66:1747-1761. https://doi.org/10.1016/j.jacc.2015.07.075
23. Feldman D, Pamboukian SV, Teuteberg JJ, Birks E, Lietz K, Moore SA, Morgan JA, Arabia F, Bauman ME, Buchholz HW, Deng M, Dickstein ML, El-Banayosy A, Elliot T, Goldstein DJ, Grady KL, Jones K, Hryniewicz K, John R, Kaan A, Kusne S, Loebe M, Massicotte MP, Moazami N, Mohacsi P, Mooney M, Nelson T, Pagani F, Perry W, Potapov EV, Rame EJ, Russell SD, Sorensen EN, Sun B, Strueber M, Mangi AA, Petty MG, Rogers J, International Society for Heart and Lung Transplantation (2013) The 2013 International Society for Heart and Lung Transplantation Guidelines for mechanical circulatory support: executive summary. J Heart Lung Transplant 32:157-187. https://doi.org/10. 1016/j.healun.2012.09.013

24. Flachskampf FA, Badano L, Daniel WG, Feneck RO, Fox KF, Fraser AG, Pasquet A, Pepi M, Perez de Isla L, Zamorano JL, European Association of Echocardiography, Echo Committee of the European Association of Cardiothoracic Anaesthesiologists, Roelandt JR, Piérard L (2010) Recommendations for transoesophageal echocardiography: update 2010. Eur J Echocardiogr 11:557-576. https://doi. org/10.1093/ejechocard/jeq057

25. Flachskampf FA, Wouters PF, Edvardsen T, Evangelista A, Habib G, Hoffman P, Hoffmann R, Lancellotti P, Pepi M (2014) Recommendations for transoesophageal echocardiography: EACVI update 2014. Eur Heart J Cardiovasc Imaging 15:353-365. https://doi.org/10.1093/ehjci/jeu015

26. Garbi M, Chambers J, Vannan MA, Lancellotti P (2015) Valve stress echocardiography: a practical guide for referral, procedure, reporting, and clinical implementation of results from the HAVEC group. JACC Cardiovasc Imaging 8:724-736. https://doi. org/10.1016/j.jcmg.2015.02.010

27. Geyer M, Sotiriou E, Tamm AR, Ruf TF, Kreidel F, Yang Y, Emrich T, Beiras-Fernandez A, Gori T, Münzel T, SchulzE, von Bardeleben RS (2019) Advanced protocol for three-dimensional transesophageal echocardiography guidance implementing realtime multiplanar reconstruction for transcatheter mitral valve repair by direct annuloplasty. J Am Soc Echocardiogr 32:1359-1365. https://doi.org/10. 1016/j.echo.2019.05.015

28. Gheorghe L, Brouwer J, Gutierrez A, Rensing BJWM, Rana BS, Ten Berg JM, Eefting FD, Timmers L, Swaans MJ (2020) Can TAVR be effectively and safely performed without Intraprocedural TEE? Curr Cardiol Rep 22:80. https://doi.org/10.1007/ s11886-020-01344-8

29. Gheorghe LL, Mobasseri S, Agricola E, Wang DD, Milla F, Swaans M, Pandis D, Adams DH, Yadav P, Sievert H, Ailawadi G, Sorajja P (2021) Imaging for native mitral valve surgical and transcatheter interventions. JACC Cardiovasc Imaging 14:112-127. https://doi.org/10.1016/j.jcmg.2020.11.021

30. Giallauria F, Di Lorenzo A, Parlato A, Testa C, Bobbio E, Vigorito C, Coats AJS (2020) Individual patient data meta-analysis of the effects of the CARILLON $^{\circledR}$ mitral contour system. ESC Heart Fail 7:3383-3391. https://doi.org/10.1002/ehf2.13125

31. Grinstein J, Kruse E, Sayer G, Fedson S, Kim GH, Jorde UP, JuricekC, Ota T, Jeevanandam V, Lang RM, Uriel N (2016) Accurate quantification methods for aortic insufficiency severity in patients with LVAD: role of diastolic flow acceleration and systolic-todiastolic peak velocity ratio of outflow cannula. JACC Cardiovasc Imaging 6:641-651. https://doi. org/10.1016/j.jcmg.2015.06.020

32. Hagendorff A, Stoebe S, Tayal B (2018) A systematic approach to 3D echocardiographic assessment of the aortic root. Glob Cardiol Sci Pract. https://doi. org/10.21542/gcsp.2018.12 
33. Hagendorff A, Evangelista A, Fehske W, Schäfers HJ (2019) Improvement in the assessment of aortic valve and aortic aneurysm repair by 3-dimensional echocardiography. JACC Cardiovasc Imaging 12:2225-2244. https://doi.org/10.1016/j.jcmg. 2018.06.032

34. Hagendorff A, Fehske W, FlachskampfFA, Helfen A, Kreidel F, Kruck S, La Rosée K, Tiemann K, Voigt JU, von Bardeleben RS, Zahn R, KnebelF (2020) Manual zur Indikation und Durchführung der Echokardiografie - Update 2020 der Deutschen Gesellschaft für Kardiologie. Kardiologe 14:396-431. https:// doi.org/10.1007/s12181-020-00402-3

35. Hagendorff A, Knebel F, Helfen A, Knierim J, Sinning C, Stöbe S, Fehske W, Ewen S (2020) Expert consensus document on the assessment of the severity of aortic valve stenosis by echocardiography to provide diagnostic conclusiveness by standardized verifiable documentation. Clin Res Cardiol 109:271-288. https://doi.org/10.1007/ s00392-019-01539-2

36. Hagendorff A, Metze M, Neef M, Jurisch D, Stress Echocardiography SS (2020) doi https://doi.org. Aktuel Kardiol. https://doi.org/10.1055/a-10074373

37. Hagendorff A, Knebel F, Helfen A, Stöbe S, Haghi D, RufT, LavallD, Knierim J, AltiokE, BrandtR, MerkeN, Ewen S (2021) Echocardiographic assessment of mitral regurgitation - discussion of practical and methodologic aspects of severity quantification to improve diagnostic conclusiveness. Clin Res Cardiol. https://doi.org/10.1007/s00392-02101841-y

38. Hahn RT, Abraham T, Adams MS, Bruce CJ, Glas KE, Lang RM, Reeves ST, Shanewise JS, Siu SC, StewartW, PicardMH(2013) Guidelines for performing a comprehensive transesophageal echocardiographic examination: recommendations from the American Society of Echocardiography and the Society of Cardiovascular Anesthesiologists. J Am Soc Echocardiogr 26:921-964. https://doi.org/10. 1016/j.echo.2013.07.009

39. Hahn RT, Little SH, Monaghan MJ, Kodali SK, Williams M, Leon MB, Gillam LD (2015) Recommendations for comprehensive intraprocedural echocardiographic imaging during TAVR. JACC Cardiovasc Imaging 8:261-287. https://doi.org/ 10.1016/j.jcmg.2014.12.014

40. Hahn RT, Nabauer M, Zuber M, Nazif TM, Hausleiter J, Taramasso M, Pozzoli A, George I, Kodali S, Bapat V, Maisano F (2019) Intraprocedural imaging of transcatheter tricuspidvalveinterventions.JACC Cardiovasc Imaging 12:532-553. https://doi.org/ 10.1016/j.jcmg.2018.07.034

41. Hahn RT, Rubenson D, Mackensen GB, Saric $M$ Little SH, Mahmood F, Cole SP, Quader N (2020) Interventional echocardiography: a new specialty interest group for American Society of Echocardiography. J Am Soc Echocardiogr 33:A10-A11. https://doi.org/10.1016/j.echo.2020. 10.004

42. Han Y, Zhang X, Zhang F (2020) Patent foramen ovale closure by using transesophageal echocardiography for cryptogenic stroke: single center experience in 132 consecutive patients. J Cardiothorac Surg 15:11. https://doi.org/10.1186/ s13019-020-1042-4

43. Izumo M, Suzuki K, Moonen M, Kou S, Shimozato T, Hayashi A, Akashi YJ, Osada N, Omiya K, Miyake F, Ohtaki E, Lancellotti P (2011) Changes in mitral regurgitation and left ventricular geometry during exercise affect exercise capacity in patients with systolic heart failure. Eur J Echocardiogr 12:54-60. https://doi.org/10.1093/ejechocard/jeq105
44. Jasic-Szpak E, Marwick TH, Donal E, PrzewlockaKosmala M, Huynh Q, Gozdzik A, Woznicka AK, Jankowska EA, Ponikowski P, Kosmala W (2021) Prediction of $A F$ in heart failure with preserved ejection fraction: incremental value of left atrial strain. JACC Cardiovasc Imaging 14:131-144. https://doi.org/10.1016/j.jcmg.2020.07.040

45. Jeon DS, Luo H, Iwami T, Miyamoto T, Brasch AV, Mirocha J, Naqvi TZ, Siegel RJ (2002) The usefulness of a $10 \%$ air- $10 \%$ blood- $80 \%$ saline mixture for contrast echocardiography: doppler measurement of pulmonary artery systolic pressure. JAm Coll Cardiol 39:124-129. https://doi. org/10.1016/s0735-1097(01)01698-9

46. Kamperidis V, van Wijngaarden SE, van Rosendael PJ, Kong WK, Leung M, Sianos G, Ajmone Marsan N, Delgado V, Bax JJ (2017) Restrictive mitral valve annuloplasty: prognostic implications of left ventricular forward flow. Ann Thorac Surg 104:1464-1470. https://doi.org/10.1016/j. athoracsur.2017.05.073

47. Kislitsina ON, Cox JL, Shah SJ, Malaisrie SC, Kruse J, Liu M, Andrei AC, McCarthy PM (2020) Preoperative left atrial strain abnormalities are associated with the development of postoperative atrial fibrillation following isolated coronary artery bypass surgery. J Thorac Cardiovasc Surg. https://doi.org/10.1016/ j.jtcvs.2020.09.130

48. Knierim J, Heck R, Pieri M, Schoenrath F, Soltani S, Stawowy P, Dreysse S, Stein J, Müller M, Mulzer J, Dandel M, Falk V, Krabatsch T, Potapov E (2019) Outcomes from a recovery protocol for patients with continuous-flowleft ventricularassist devices. J Heart Lung Transplant 38:440-448. https://doi. org/10.1016/j.healun.2018.11.001

49. Kuck KH, Bleiziffer $S$, Eggebrecht $H$, Ensminger $S$, Frerker C, Möllmann H, Nef H, Thiele H, Treede H, Wimmer-GreineckerG, WaltherT(2020)Consensus paper of the German Cardiac Society (DGK) and the German Society for Thoracic and Cardiovascular Surgery (DGTHG) on transcatheter aortic valve implantation (TAVI) 2020. Kardiologe 14:182-204. https://doi.org/10.1007/s12181-020-00398-w

50. Kusunose K, Popović ZB, Motoki H, Marwick TH (2013) Prognostic significance of exercise-induced right ventricular dysfunction in asymptomatic degenerative mitral regurgitation. Circ Cardiovasc Imaging 6:167-176. https://doi.org/10.1161/ CIRCIMAGING.112.000162

51. Lancellotti P, Gérard PL, Piérard LA (2005) Longterm outcome of patients with heart failure and dynamic functional mitral regurgitation. Eur Heart J 26:1528-1532. https://doi.org/10.1093/ eurheartj/ehi189

52. Lancellotti P, Lebois F, Simon M, Tombeux C, Chauvel C, Pierard LA (2005) Prognostic importance of quantitative exercise Dopplerechocardiography in asymptomatic valvular aortic stenosis. Circulation 112(Suppl):I377-I382. https://doi.org/10.1161/ CIRCULATIONAHA.104.523274

53. Lancellotti P, Cosyns B, Zacharakis D, Attena E, Van Camp G, Gach O, Radermecker M, Piérard LA (2008) Importance of left ventricular longitudina function and functional reserve in patients with degenerative mitral regurgitation: assessment by two-dimensional speckle tracking. J Am Soc Echocardiogr 21:1331-1336. https://doi.org/10. 1016/j.echo.2008.09.023

54. Lancellotti P, Karsera D, Tumminello G, Lebois F, Piérard LA (2008) Determinants of an abnormal response to exercise in patients with asymptomatic valvular aortic stenosis. Eur J Echocardiogr 9:338-343. https://doi.org/10.1016/j.euje.2007. 04.005
55. Lancellotti P, Magne J, Donal E, O'Connor K, Dulgheru R, Rosca M, Pierard LA (2012) Determinants and prognostic significance of exercise pulmonary hypertension in asymptomatic severe aortic stenosis. Circulation 126:851-859. https:// doi.org/10.1161/CIRCULATIONAHA.111.088427

56. Lancellotti P, Magne J (2013) Stress echocardiography in regurgitant valve disease. Circ Cardiovasc Imaging 6:840-849. https://doi.org/10.1161/ CIRCIMAGING.113.000474

57. Lancellotti P, Fattouch K, La Canna G (2015) Therapeutic decision-making for patients with fluctuating mitral regurgitation. Nat Rev Cardiol 12:212-219. https://doi.org/10.1038/nrcardio. 2015.16

58. Lancellotti P, Pellikka PA, Budts W, Chaudhry FA, Donal E, Dulgheru R, Edvardsen T, Garbi M, Ha JW, Kane GC, Kreeger J, Mertens L, Pibarot P, Picano E, Ryan T, Tsutsui JM, Varga A (2016) The clinical use of stress echocardiography in non-ischaemic heart disease: recommendations from the European Association of Cardiovascular Imaging and the American Society of Echocardiography. Eur Heart J Cardiovasc Imaging 17:1191-1229. https://doi. org/10.1093/ehjci/jew190

59. Lancellotti P, Pibarot $P$, Chambers J, Edvardsen $T$, Delgado V, Dulgheru R, Pepi M, Cosyns B, Dweck MR, Garbi M, Magne J, Nieman K, Rosenhek R, Bernard A, Lowenstein J, Vieira ML, Rabischoffsky A, Vyhmeister RH, Zhou X, Zhang Y, Zamorano JL, Habib G (2016) Recommendations for the imaging assessment of prosthetic heart valves: a report from the European Association of Cardiovascular Imaging endorsed by the Chinese Society of Echocardiography, the Inter-American Society of Echocardiography, and the Brazilian Department of Cardiovascular Imaging. Eur Heart J Cardiovasc Imaging 17:589-590. https://doi.org/ 10.1093/ehjci/jew025

60. Lang RM, Badano LP, Tsang W, Adams DH, Agricola E, Buck T, Faletra FF, Franke A, Hung J, de Isla LP, Kamp O, Kasprzak JD, Lancellotti P, Marwick TH, McCulloch ML, Monaghan MJ, Nihoyannopoulos P, Pandian NG, Pellikka PA, Pepi M, Roberson DA, Shernan SK, Shirali GS, Sugeng L, Ten CFJ, Vannan MA, Zamorano JL, Zoghbi WA, American Society of Echocardiography, European Association of Echocardiography (2012) EAE/ASE recommendations for image acquisition and display using threedimensional echocardiography. Eur Heart J Cardiovasc Imaging 13:1-46. https://doi.org/10.1093/ ehjci/jer316

61. Lang RM, Badano LP, Mor-Avi V, Afilalo J, Armstrong A, Ernande L, Flachskampf FA, Foster E, Goldstein SA, Kuznetsova T, Lancellotti P, Muraru D, Picard MH, Rietzschel ER, Rudski L, Spencer KT, Tsang W, Voigt JU (2015) Recommendations for cardiac chamber quantification by echocardiography in adults: an update from the American Society of Echocardiography and the European Association of Cardiovascular Imaging. Eur Heart J Cardiovasc Imaging 16:233-270. https://doi.org/ 10.1093/ehjci/jev014

62. Leong DP, Joyce $E$, Debonnaire $P$, Katsanos $S$, Holman ER, Schalij MJ, Bax JJ, Delgado V, Marsan NA (2017) Left atrial dysfunction in the pathogenesis of cryptogenic stroke: novel insights from speckle-tracking echocardiography. J Am Soc Echocardiogr 30:71-79.e1. https://doi.org/10. 1016/j.echo.2016.09.013

63. Li W, West C, McGhie J, van den Bosch AE, BabuNarayan SV, Meijboom F, Mongeon FP, Khairy P, Kimball TR, Beauchesne LM, Ammash NM, Veldtman GR, Oechslin E, Gatzoulis MA, Webb G (2018) 
Consensus recommendations for echocardiography in adults with congenital heart defects from the International Society of Adult Congenital Heart Disease (ISACHD). Int J Cardiol 272:77-83. https:// doi.org/10.1016/j.ijcard.2018.07.058

64. Mackensen GB, Lee JC, Wang DD, Pearson PJ, Blanke P, Dvir D, Kirkpatrick JN (2018) Role of echocardiography in transcatheter mitral valve replacement in native mitral valves and mitral rings. J Am Soc Echocardiogr 31:475-490. https:// doi.org/10.1016/j.echo.2018.01.011

65. Magne J, Lancellotti P, Piérard LA (2010) Exerciseinduced changes in degenerative mitral regurgitation. J Am Coll Cardiol 56:300-309. https://doi.org/ 10.1016/j.jacc.2009.12.073

66. Mandoli GE, Cameli M, Novo G, Agricola E, Righini FM, Santoro C, D'Ascenzi F, Ancona F, Sorrentino R, D'Andrea A, Galderisi M, Mondillo S, Working Group of Echocardiography of the Italian Society of Cardiology (2019) Right ventricular function after cardiac surgery: the diagnostic and prognostic role of echocardiography. Heart Fail Rev 24:625-635. https://doi.org/10.1007/s10741019-09785-2

67. Maréchaux S, Hachicha Z, Bellouin A, Dumesnil JG, Meimoun P, Pasquet A, Bergeron $S$, Arsenault M, Le Tourneau T, Ennezat PV, PibarotP (2010) Usefulness of exercise-stress echocardiography for risk stratification of true asymptomatic patients with aortic valve stenosis. Eur Heart J 31:1390-1397. https://doi.org/10.1093/eurheartj/ehq076

68. McCarthy PM, Herborn J, Kruse J, Liu M, Andrei AC, Thomas JD (2020) A multiparameter algorithm to guide repair of degenerative mitral regurgitation. JThorac Cardiovasc Surg. https://doi.org/10.1016/ j.jtcvs.2020.09.129

69. Medvedofsky D, Mor-Avi V, Amzulescu M, Fernández-Golfín C, Hinojar R, Monaghan MJ, Otani K, Reiken J, Takeuchi M, Tsang W, Vanoverschelde JL, Indrajith $\mathrm{M}$, Weinert L, Zamorano JL, Lang RM (2018) Three-dimensional echocardiographic quantification of the left-heart chambers using an automated adaptive analytics algorithm: multicentre validation study. Eur Heart J Cardiovasc Imaging 19:47-58. https://doi.org/10.1093/ehjci/ jew328

70. Mehra MR, Uriel N, Naka Y, Cleveland JC Jr, Yuzefpolskaya M, Salerno CT, Walsh MN, Milano CA, Patel CB, Hutchins SW, Ransom J, Ewald GA, Itoh A, Raval NY, Silvestry SC, Cogswell R, John $R$, Bhimaraj A, Bruckner BA, Lowes BD, Um JY, Jeevanandam V, Sayer G, Mangi AA, Molina EJ, Sheikh F, Aaronson K, Pagani FD, Cotts WG, Tatooles AJ, Babu A, Chomsky D, Katz JN, Tessmann PB, Dean D, Krishnamoorthy A, Chuang J, Topuria I, Sood P, Goldstein DJ, MOMENTUM 3 Investigators (2019) A fully magnetically levitated left ventricular assist device - final report. $N$ Engl J Med 380:1618-1627. https://doi.org/10.1056/ NEJMoa1900486

71. Messika-Zeitoun D, Nickenig G, Latib A, Kuck KH, Baldus S, Schueler R, La Canna G, Agricola E, Kreidel $F$, Huntgeburth $M$, Zuber $M$, Verta $P$, Grayburn P, Vahanian A, Maisano F (2019) Transcatheter mitral valve repair for functional mitral regurgitation using the Cardioband system: 1 year outcomes. Eur Heart J 40:466-472. https:// doi.org/10.1093/eurheartj/ehy424

72. Miglioranza MH, Badano LP, Mihăilă S, Peluso D, Cucchini U, Soriani N, lliceto S, Muraru D (2016) Physiologic determinants of left atrial longitudinal strain: a two-dimensional speckletracking and three-dimensional echocardiographic study in healthy volunteers. J Am Soc
Echocardiogr 29:1023-1034.e3. https://doi.org/ 10.1016/j.echo.2016.07.011

73. Mihăilă S, Muraru D, Piasentini E, Miglioranza MH, Peluso D, Cucchini U, lliceto S, Vinereanu D, Badano LP (2014) Quantitative analysis of mitral annular geometry and function in healthy volunteers using transthoracic three-dimensional echocardiography. J Am Soc Echocardiogr 27:846-857. https:// doi.org/10.1016/j.echo.2014.04.017

74. Mur MJL, García MA, García Lledó A, Rivera LC, Rincón Díaz LM, Miguelena Hycka J, Boretti I, Gimaraes C, Casas Rojo E, Jiménez Nacher JJ, FernándezGolfín C, Rodríguez-Roda SJ, Zamorano JL (2018) Geometrical and functional cardiac changes after cardiac surgery: a physiopatological explanation based on speckle tracking. Int J Cardiovasc Imaging 34:1905-1915. https://doi.org/10.1007/s10554018-1424-z

75. Muraru D, Cattarina M, Boccalini F, Dal Lin C, Peluso D, Zoppellaro G, Bellu R, Sarais C, Xhyheri B, lliceto S, Badano LP (2013) Mitral valve anatomy andfunction:newinsights from three-dimensional echocardiography. J Cardiovasc Med 14:91-99. https://doi.org/10.2459/JCM.0b013e328356a577

76. Muraru D, Hahn RT, Soliman OI, Faletra FF, Basso C, Badano LP (2019) 3-dimensional echocardiography in imaging the tricuspid valve. JACC CardiovasC Imaging 12:500-515. https://doi.org/10.1016/j. jcmg.2018.10.035

77. Muskula PR, Main ML (2017) Safety with echocardiographic contrast agents. Circ Cardiovasc Imaging 10:e5459. https://doi.org/10.1161/ CIRCIMAGING.116.005459

78. Nickenig G, Weber M, Schüler R, Hausleiter J, Nabauer M, von Bardeleben RS, Sotiriou $E$, Schäfer U, Deuschl F, Alessandrini H, Kreidel F, Juliard JM, Brochet E, Latib A, Montorfano M, Agricola E, Baldus S, Friedrichs KP, Deo $\mathrm{SH}_{\text {, }}$ Gilmore SY, Feldman T, Hahn RT, Maisano F (2021) Tricuspid valve repair with the Cardioband system: two-year outcomes of the multicentre, prospective TRI-REPAIR study. Eurolntervention 16:e1264-e1271. https://doi.org/10.4244/EIJ-D20-01107

79. Nishimura RA, Otto CM, Bonow RO, Carabello BA Erwin JP 3rd, Fleisher LA, Jneid $H$, Mack MJ, McLeod CJ, O'Gara PT, Rigolin VH, Sundt TM 3rd, Thompson A (2017) 2017 AHA/ACC Focused Update of the 2014 AHA/ACC Guideline for the Management of Patients with Valvular Heart Disease: A Report of the American College of Cardiology/American Heart Association TaskForce on Clinical Practice Guidelines. J Am Coll Cardiol 70:252-289. https://doi.org/10.1016/j.jacc.2017. 03.011

80. Nicoara A, Skubas N, Ad N, Finley A, Hahn RT, Mahmood F, Mankad S, Nyman CB, Pagani F, Porter TR, Rehfeldt K, Stone M, Taylor B, Vegas A, Zimmerman KG, Zoghbi WA, Swaminathan M (2020) Guidelines for the use of transesophageal echocardiography to assist with surgical decisionmaking in the operating room: a surgerybased approach: from the American Society of Echocardiographyin collaboration with the society of cardiovascular anesthesiologists and the society of thoracic surgeons. J Am Soc Echocardiogr 33:692-734. https://doi.org/10.1016/j.echo.2020. 03.002

81. Okai T, Mizutani K, Hara M, Yamaguchi $T$, Ogawa M, Ito A, Iwata S, Izumiya Y, Takahashi Y, Shibata T, Yoshiyama M (2020) Presence of mitra stenosis is a risk factor of new development of acute decompensated heart failure early after transcatheter aortic valve implantation. Open
Heart 7:e1348. https://doi.org/10.1136/openhrt2020-001348

82. Otto CM, Nishimura RA, Bonow RO, Carabello BA, Erwin JP, Gentile F, Jneid $H$, Krieger EV, Mack $M$, McLeod C, O'Gara PT, Rigolin VH, Sundt TM, Thompson A, Toly C (2021) 2020 ACC/AHA guideline for the management of patients with valvular heart disease: a report of the American College of Cardiology/American heart association joint committee on clinical practice guidelines. J Am Coll Cardiol 77:e25-e197. https://doi.org/10. 1016/j.jacc.2020.11.018

83. Pathan F, D'Elia N, Nolan MT, Marwick TH, Negishi K (2017) Normal ranges of left atrial strain by speckle-tracking echocardiography: a systematic review and meta-analysis. J Am Soc Echocardiogr 30:59-70.e8.https://doi.org/10.1016/j.echo.2016. 09.007

84. Pellikka PA, Arruda-Olson A, Chaudhry FA, Chen MH, Marshall JE, Porter TR, Sawada SG (2020) Guidelines for performance, interpretation, and application of stress echocardiography in Ischemic heart disease: from the American Society of Echocardiography. J Am Soc Echocardiogr 33:1-41.e8. https://doi.org/10.1016/j.echo.2019. 07.001

85. Peyrou J, Chauvel C, Pathak A, Simon M, Dehant P, Abergel E (2017) Preoperative right ventricular dysfunction is a strong predictor of 3 years survival aftercardiacsurgery. Clin Res Cardiol 106:734-742. https://doi.org/10.1007/s00392-017-1117-y

86. Piayda K, Hellhammer K, Nielsen-Kudsk JE, Schmidt B, Mazzone P, Berti S, Fischer S, Lund J, Montorfano M, Bella DP, Gage R, Zeus T (2021) Clinical outcomes of patients undergoing percutaneous left atrial appendage occlusion in general anaesthesia or conscious sedation: data from the prospective global Amplatzer Amulet Occluder Observational Study. BmjOpen 11:e40455.https:// doi.org/10.1136/bmjopen-2020-040455

87. Pibarot $P$, Dumesnil JG, Jobin J, Lemieux M, Honos G, Durand LG (1999) Usefulness of the indexed effective orifice area at rest in predicting an increase in gradient during maximum exercise in patients with a bioprosthesis in the aortic valve position. Am J Cardiol 83:542-546. https://doi.org/ 10.1016/s0002-9149(98)00910-2

88. Picano E, Pibarot P, Lancellotti P, Monin JL, Bonow RO (2009) The emerging role of exercise testing and stress echocardiography in valvular heart disease. J Am Coll Cardiol 54:2251-2260. https://doi.org/10.1016/j.jacc.2009.07.046

89. Pibarot P, Hahn RT, Weissman NJ, Monaghan MJ (2015) Assessment of paravalvular regurgitation following TAVR: a proposal of unifying grading scheme. JACC Cardiovasc Imaging 8:340-360. https://doi.org/10.1016/j.jcmg.2015.01.008

90. PorterTR, Mulvagh SL, Abdelmoneim SS, Becher H, Belcik JT, Bierig M, Choy J, Gaibazzi N, Gillam LD, Janardhanan R, Kutty S, Leong-Poi H, Lindner JR, Main ML, Mathias W Jr, Park MM, Senior R, Villanueva F (2018) Clinical applications of ultrasonic enhancing agents in echocardiography: 2018 American Society of Echocardiography guidelines update. J Am Soc Echocardiogr 31:241-274. https://doi.org/10.1016/j.echo.2017. 11.013

91. Prihadi EA, van Rosendael PJ, Vollema EM, Bax JJ, Delgado V, Ajmone Marsan N (2018) Feasibility, accuracy, and reproducibility of aortic annular and root sizing for transcatheter aortic valve replacement using novel automated three-dimensional Echocardiographic software: comparison with multi-detector row computed Tomography. J Am 
Soc Echocardiogr 31:505-514.e3. https://doi.org/ 10.1016/j.echo.2017.10.003

92. Raina A, Vaidya A, Gertz ZM, Chambers S, Forfia PR (2013) Marked changes in right ventricular contractile pattern after cardiothoracic surgery: implications for post-surgical assessment of right ventricular function. J Heart Lung Transplant 32:777-783. https://doi.org/10.1016/j.healun. 2013.05.004

93. Ramsingh D, Hu H, Yan M, Lauer R, Rabkin D, Gatling J, Floridia R, Martinez M, Dorotta I, Razzouk A (2021) Perioperative individualized goal directed therapy for cardiac surgery: a historical-prospective, comparative effectiveness study. J Clin Med 10:400. https://doi.org/10.3390/ jcm10030400

94. Rana BS, Thomas MR, Calvert PA, Monaghan MJ, Hildick-Smith D (2010) Echocardiographic evaluation of patent foramen ovale prior to device closure. JACC Cardiovasc Imaging 3:749-760. https://doi.org/10.1016/j.jcmg.2010.01.007

95. Rana BS (2018) Echocardiography guidance of atrial septal defect closure. J Thorac Dis 10(Suppl 24):S2899-S2908. https://doi.org/10.21037/jtd. 2018.07.126

96. Reis G, Motta MS, Barbosa MM, Esteves WA, Souza SF, Bocchi EA (2004) Dobutamine stress echocardiography for noninvasive assessment and risk stratification of patients with rheumatic mitral stenosis. J Am Coll Cardiol 43:393-401. https://doi. org/10.1016/j.jacc.2003.09.037

97. Rogers JG, Pagani FD, Tatooles AJ, Bhat G, Slaughter MS, Birks EJ, Boyce SW, Najjar SS, Jeevanandam V, Anderson AS, Gregoric ID, Mallidi $\mathrm{H}$, Leadley $\mathrm{K}$, Aaronson KD, Frazier $\mathrm{OH}$, Milano CA (2017) Intrapericardial left ventricular assist device for advanced heart failure. $\mathrm{N}$ Engl J Med 376:451-460. https://doi.org/10.1056/ NEJMoa 1602954

98. Scandroglio AM, Kaufmann F, Pieri M, Kretzschmar A, Müller M, Pergantis P, Dreysse S, Falk V, Krabatsch T, Potapov EV (2016) Diagnosis and treatment algorithm for blood flow obstructions in patients with left ventricular assist device. J Am Coll Cardiol 67:2758-2768. https://doi.org/10.1016/j. jacc.2016.03.573

99. Senior R, Becher H, Monaghan M, Agati L, Zamorano J, Vanoverschelde JL, Nihoyannopoulos P, Edvardsen T, Lancellotti P, EACVI Scientific Documents Committeefor 2014-16and2016-18(2017) Clinical practice of contrast echocardiography: recommendation by the European Association of Cardiovascular Imaging (EACVI) 2017. Eur Heart J Cardiovasc Imaging 18:1205-1205a. https://doi. org/10.1093/ehjic/jex182

100. Sicari R, Nihoyannopoulos P, Evangelista A, Kasprzak J, Lancellotti P, Poldermans D, Voigt JU, Zamorano JL, European Association of Echocardiography (2009) Stress echocardiography expert consensus statement-executive summary: European Association of Echocardiography (EAE) (a registered branch of the ESC). Eur Heart J 30:278-289. https://doi.org/10.1093/eurheartj/ ehn492

101. Silvestry FE, Kerber RE, Brook MM, Carroll JD, Eberman KM, Goldstein SA, Herrmann HC, Homma S, Mehran R, Packer DL, Parisi AF, Pulerwitz T, Seward JB, Tsang TS, Wood MA (2009) Echocardiography-guided interventions. J Am Soc Echocardiogr 22:213-231. https://doi.org/10. 1016/j.echo.2008.12.013 (quiz316)

102. Sorajja P, Moat N, Badhwar V, Walters D, Paone G, Bethea B, Bae R, Dahle G, Mumtaz M, Grayburn P, Kapadia S, Babaliaros V, Guerrero M, Satler L,

\section{Manual on indications and performance of specific echocardiographic applications}

The second manual on indications for and performance of echocardiography focusses on specific echocardiographic modalities and special problems in several patient cohorts. Practical aspects are discussed with priority. Established echocardiographic modalities are transesophageal, stress and contrast echocardiography. Modern technologies, such as 3D echocardiography and deformation imaging play an important role in nearly all scenarios of clinical echocardiography. This complete echocardiographic spectrum is incorporated into emergency and intensive care medicine, monitoring interventions of structural heart diseases, cardiac surgery, patients with cardiac assist devices, adult patients with congenital heart diseases and the care of highly infectious patients in pandemics. The diagnostic procedures of conventional and modern echocardiography are highlighted in this manual. The use of 3D echocardiography to characterize cardiac morphology and the application of deformation imaging to objectify cardiac function are already established in clinical practice. Stress echocardiography to detect myocardial ischemia and viability or to diagnose valvular heart diseases, assessment of coronary flow reserve to analyze myocardial perfusion and contrast echocardiography for left ventricular opacification and tumor characterization are increasingly being used to improve diagnostics. As mentioned for conventional echocardiography in the last manual of echocardiography in 2009 , the modern features in echocardiography require standardized documentation and acquisition of certain images with optimized ultrasound settings, because conclusive and reproducible data analysis can only be performed if the image quality is sufficient.

\section{Keywords}

Transesophageal echocardiography - Stress echocardiography · Contrast echocardiography . Deformation imaging · Echocardiographic monitoring

Thourani V, Bedogni F, RizikD, Denti P, Dumonteil N, Modine T, Sinhal A, Chuang ML, Popma JJ, Blanke P, Leipsic J, Muller D (2019) Initial feasibility study of a new transcatheter mitral prosthesis: the first 100 patients. J Am Coll Cardiol 73:1250-1260. https:// doi.org/10.1016/j.jacc.2018.12.066

103. Stainback RF, Estep JD, Agler DA, Birks EJ, Bremer M, Hung J, Kirkpatrick JN, Rogers JG, Shah NR, American Society of Echocardiography (2015) Echocardiography in the management of patients with left ventricular assist devices: recommendations from the American Society of Echocardiography. J Am Soc Echocardiogr 28:853-909. https://doi.org/10.1016/j.echo.2015. 05.008

104. Steeds RP, Wheeler R, Bhattacharyya S, Reiken J, Nihoyannopoulos P, Senior R, Monaghan MJ, Sharma V (2019) Stress echocardiography in coronary artery disease: a practical guideline from the British Society of Echocardiography. Echo Res Pract 6:G17-G33. https://doi.org/10.1530/ERP18-0068

105. Stella S, Italia L, Geremia G, Rosa I, Ancona F, Marini C, Capogrosso C, Giglio M, Montorfano M, Latib A, Margonato A, Colombo A, Agricola E (2019) Accuracy and reproducibility of aortic annular measurements obtained from echocardiographic 3D manual and semi-automated software analyses in patients referred for transcatheter aortic valve implantation: implication for prosthesis size selection. Eur Heart J Cardiovasc Imaging 20:45-55. https://doi.org/10.1093/ehjci/jey013
106. Stephens RS, Whitman GJ (2015) Postoperative critical care of the adult cardiac surgical patient. Part I: routine postoperative care. Crit Care Med 43:1477-1497. https://doi.org/10.1097/CCM. 0000000000001059

107. Stöbe S, Richter S, Seige M, Stehr S, Laufs U, Hagendorff A (2020) Echocardiographic characteristics of patients with SARS-CoV-2 infection. Clin Res Cardiol 109:1549-1566. https://doi.org/10.1007/ s00392-020-01727-5

108. Suzuki K, Izumo M, Yoneyama K, Mizukoshi K, Kamijima R, Kou S, Takai M, Kida K, Watanabe S, Omiya K, Nobuoka S, Akashi YJ (2015) Influence of exercise-induced pulmonary hypertension on exercise capacity in asymptomatic degenerative mitral regurgitation.J Cardiol 66:246-252. https:// doi.org/10.1016/j.jjcc.2014.11.005

109. Teuteberg JJ, Cleveland JC Jr, Cowger J, Higgins RS, Goldstein DJ, Keebler M, Kirklin JK, Myers SL, Salerno CT, Stehlik J, Fernandez F, Badhwar V, Pagani FD, Atluri P (2020) The society of thoracic surgeons Intermacs 2019 annual report: the changing landscape of devices and indications. Ann Thorac Surg 109:649-660. https://doi.org/10. 1016/j.athoracsur.2019.12.005

110. Truby LK, Garan AR, Givens RC, Wayda B, Takeda K, Yuzefpolskaya M, Colombo PC, Naka Y, Takayama H, Topkara VK (2018) Aortic insufficiency during contemporary left ventricular assist device support: analysis of the INTERMACS registry. JACC Heart Fail 11:951-960. https://doi.org/10.1016/j. jchf.2018.07.012 
111. Uhlig J, Al-Bourini O, Salgado R, Francone $M$, Vliegenthart R, Bremerich J, Lotz J, Gutberlet M (2020) Gadolinium-based contrast agents for cardiac MRI: use of linear and macrocyclic agents with associated safety profile from 154779 European patients. Radiol Cardiothorac Imaging 2:5.https://doi.org/10.1148/ryct.2020200102

112. Vahanian A, Brochet E (2017) Transseptal puncture for structural heart intervention: an old technique with new indications. Heart 103:1830-1837. https://doi.org/10.1136/heartjnl-2016-310483

113. Varghese $R$, Itagaki S, Anyanwu AC, Milla F, Adams DH (2014) Predicting early left ventricular dysfunction after mitral valve reconstruction: the effect of atrial fibrillation and pulmonary hypertension. J Thorac Cardiovasc Surg 148:422-427. https://doi.org/10.1016/j.jtcvs.2013.08.073

114. Voigt JU, Pedrizzetti G, Lysyansky P, Marwick TH, Houle H, Baumann R, Pedri S, Ito Y, Abe Y, Metz S, Song JH, Hamilton J, Sengupta PP, Kolias TJ, d'Hooge J, Aurigemma GP, Thomas JD, Badano LP (2015) Definitions for a common standard for 2D speckle tracking echocardiography: consensus document of the EACVI/ASE/Industry Task Force to standardize deformation imaging. Eur Heart J Cardiovasc Imaging 16:1-11. https://doi.org/10. 1093/ehjci/jeu184

115. Werdan K, Baldus S, Bauersachs J, Baumgartner $\mathrm{H}$ Bongarth $C M$, Buerke $M$, Dörr $R$, Duncker $D_{\text {, }}$ Eckardt L, El-Armouche A, Elsässer A, Fach A, Flachskampf FA, Gabelmann M, Griebenow R, Heinemann-Meerz S, Hoffmeister HM, Katus HA, Kaul N, Krämer LI, Kuhn B, Lange T, Lehmann LH, Lugenbiel P, Michels G, Müller-Werdan U, Oldenburg O, Rittger H, Rottbauer W, Rybak K, Sack S, Skobel CE, Smetak N, Thiele H, Tiefenbacher C, Tiemann K, Voelker W, Zeiher A, Frey N (2020) Curriculum Kardiologie 2., aktualisierte Auflage. Kardiologe 14:505-536. https://doi.org/10.1007/ s12181-020-00425-w

116. Wiegers SE, Ryan T, Arrighi JA, Brown SM, Canaday B, Damp JB, Diaz-Gomez JL, Figueredo VM Garcia MJ, Gillam LD, Griffin BP, Kirkpatrick JN, Klarich KW, Lui GK, Maffett S, Naqvi TZ, Patel AR, Poulin MF, Rose GA, Swaminathan M (2019) 2019 ACC/AHA/ASE advanced training statement on echocardiography (revision of the 2003 ACC/AHA clinical competence statement on echocardiography): a report of the ACC competency management committee. J Am Coll Cardiol 74:377-402. https:// doi.org/10.1016/j.jacc.2019.02.003

117. WunderlichNC,SiegelRJ(2013) Peri-interventional echo assessment for the MitraClip procedure. Eur Heart J Cardiovasc Imaging 14:935-949. https:// doi.org/10.1093/ehjci/jet060

118. Wunderlich NC, Beigel R, Swaans MJ, Ho SY, SiegelRJ (2015) Percutaneous interventions for left atrial appendage exclusion: options, assessment, and imaging using 2D and 3D echocardiography. JACC Cardiovasc Imaging 8:472-488. https://doi. org/10.1016/j.jcmg.2015.02.002

119. Zamorano JL, Badano LP, Bruce C, Chan KL, Gonçalves A, Hahn RT, Keane MG, La Canna G, Monaghan MJ, Nihoyannopoulos P, Silvestry FE, Vanoverschelde JL, Gillam LD, Vahanian A, Di Bello V, Buck T (2011) EAE/ASE recommendations for the use of echocardiography in new transcatheter interventions for valvular heart disease. Eur JEchocardiogr 12:557-584. https://doi.org/10. 1093/ejechocard/jer086

120. Zoghbi WA, Adams D, Bonow RO, EnriquezSarano M, Foster E, Grayburn PA, Hahn RT, Han Y, Hung J, Lang RM, Little SH, Shah DJ, Shernan S, Thavendiranathan P, Thomas JD, Weissman NJ (2017)
Recommendations for noninvasive evaluation of native valvular regurgitation: a report from the American Society of Echocardiography developed in collaboration with the society for cardiovascular magnetic resonance. J Am Soc Echocardiogr 30:303-371. https://doi.org/10.1016/j.echo.2017. 01.007

121. Zoghbi WA, Asch FM, Bruce C, Gillam LD, Grayburn PA, Hahn RT, Inglessis I, Islam AM, Lerakis S, Little SH, Siegel RJ, Skubas N, Slesnick TC, Stewart WJ, Thavendiranathan P, Weissman NJ, Yasukochi S, Zimmerman KG (2019) Guidelines for the evaluation of valvular regurgitation after Percutaneous valve repair or replacement: a report from the American Society of Echocardiography developed in collaboration with the society for cardiovascular angiography and interventions, Japanese society of Echocardiography, and society for Cardiovascular Magnetic Resonance. J Am Soc Echocardiogr 32:431-475. https://doi.org/10. 1016/j.echo.2019.01.003
MED UPDATE SEMINARE 2022

Cardio Update 2022

17. DGK-Kardiologie-Update-Seminar

04.-05. März 2022

Mainz und Livestream

11.-12. März 2022

Berlin und Livestream

Wiss. Leitung:

Prof. Dr. Michael Böhm, Homburg

Prof. Dr. Stephan Achenbach, Erlangen

Prof. Dr. Ulrich Laufs, Leipzig

Prof. Dr. Thorsten Lewalter, München

Unter der Schirmherrschaft der DGK, DGIM

www.cardio-update.com

Auskunft für alle Update-Seminare: med update $\mathrm{GmbH}$ www.med-update.com

Tel.: 0611 - 736580

info@med-update.com 